\title{
FUNCTORIALITY, SMITH THEORY, AND THE BRAUER HOMOMORPHISM
}

\author{
DAVID TREUMANN AND AKSHAY VENKATESH
}

\begin{abstract}
If $\sigma$ is an automorphism of order $p$ of the semisimple group $\mathbf{G}$, there is a natural correspondence between $\bmod p$ cohomological automorphic forms on $\mathbf{G}$ and $\mathbf{G}^{\sigma}$. We describe this correspondence in the global and local settings.
\end{abstract}

\section{INTRODUCTION}

1.1. Let $\mathbf{G}$ be a semisimple group over a number field $F$, with Langlands-dual ${ }^{L} \hat{G}$, and let $k$ be an algebraically closed field of positive characteristic $p$. By "mod $p$ automorphic forms" for $\mathrm{G}$ we shall mean Hecke eigenclasses in the cohomology of congruence subgroups with $k$-coefficients. We make no assumption that these cohomology classes lift to characteristic zero, i.e. there may be no automorphic form in the classical sense associated to this eigenclass.

Now let $\sigma$ be an order $p$ automorphism of $\mathbf{G}$, defined over $F$, with a connected fixed point subgroup $\mathbf{G}^{\sigma}$. The main goal of this paper is to show that there is a close relationship between $\bmod p$ automorphic forms on $\mathbf{G}$ and $\bmod p$ automorphic forms on $\mathbf{G}^{\sigma}$ : we construct a homomorphism $(\$ 4.3)$

$$
\begin{aligned}
& \text { Hecke algebra for } \mathbf{G} \text { at } v, \stackrel{\psi_{v}}{\longrightarrow} \text { Hecke algebra for } \mathbf{G}^{\sigma} \text { at } v \text {, } \\
& \text { with } k \text { coefficients } \longrightarrow \quad \text { with } k \text { coefficients }
\end{aligned}
$$

which is a slight variant of the "Brauer homomorphism" of modular representation theory. We prove (see Theorem 5.8):

First Main Theorem. If a mod p automorphic form for $\mathbf{G}^{\sigma}$ has Satake parameters $\left\{a_{v}\right\}$ then there exists a mod $p$ automorphic form for $\mathbf{G}$ with Satake parameters $\left\{\psi_{v}^{*}\left(a_{v}\right)\right\}$.

What is the relationship between the parameters of these forms at ramified places? This is answered by Theorem 6.5, based on the the notion of "linkage" (Definition 6.2) of local representations. Roughly speaking, these results suggest that local functoriality should be realized by Tate cohomology.

One of course wants to compute $\psi_{v}$. This is accomplished by the Theorem of $\S 8.1$. The formulation in $\$ 8.1$ is not the natural one from the point of view of the Langlands program. The remainder of the paper addresses this issue, which we now describe:

1.2. Torsion functoriality. Write $\mathbf{H}=\mathbf{G}^{\sigma}$. One wants to know if the "lift" furnished by the theorem is a "functorial lift" in the sense of Langlands: is $\psi_{v}$ induced by a $L$ homomorphism of $L$-groups

$$
{ }^{L} \hat{\psi}:{ }^{L} \hat{H} \rightarrow{ }^{L} \hat{G} ?
$$

For the precise meaning of the word "induced" here, see $\$ 7.7$. In the setting of our paper, it is natural to construct the dual groups as algebraic groups over $k$, rather than over $\mathbf{C}$, and we shall always follow that convention $(\$ 2.5)$. In that case the Theorem amounts to functoriality for $\psi$ and we call such an ${ }^{L} \hat{\psi}$ a $\sigma$-dual homomorphism. 
A basic example is that of cyclic base change. If $\mathbf{G}=\operatorname{Res}_{E / F}\left(\mathbf{H} \otimes_{F} E\right)$ where $E / F$ is a cyclic extension of degree $p$, and $\sigma$ induced by a generator of $\operatorname{Aut}(E / F)$, then we may take ${ }^{L} \hat{\psi}$ to be the homomorphism obtained by restriction on the Galois group, and the result amounts to "cyclic base change." It doesn't follow from usual base change, even for $\mathbf{H}=\mathrm{SL}_{2}$ and $F=\mathbf{Q}(i)$, as our result applies to characteristic $p$ torsion classes. Indeed, this result itself seems interesting: we do not know of any other examples of where functoriality can be established, in such a strong form, for torsion classes (compare [11]).

Our second main theorem verifies that such ${ }^{L} \hat{\psi}$ exists at least in some generality:

Second Main Theorem. Suppose that $\mathbf{G}$ is simply connected and $\mathbf{H}$ is semisimple ${ }^{1}$. Then there exists a $\sigma$-dual homomorphism ${ }^{L} \hat{H} \rightarrow{ }^{L} \hat{G}$ with the possible exception of cases ${ }^{2}$ where $(\operatorname{Lie}(\mathbf{G}), \operatorname{Lie}(\mathbf{H}))$ contains a factor of the form $\left(\mathfrak{e}_{6}, \mathfrak{s l}_{3}^{3}\right.$ or $\mathfrak{s l}_{6} \times \mathfrak{s l}_{2}$ or $\left.\mathfrak{s p}_{8}\right)$.

In summary, then, the two theorems together give a functoriality for $\bmod p$ automorphic forms, from $\mathbf{H}$ to $\mathbf{G}$.

We prove this second theorem using the Theorem of $\S 8.1$, some general arguments, and finally some case-by-case verification for some exceptional cases. Because the case-bycase verifications are lengthy, and the remainder of the proof is already long, we present only the most interesting cases (e.g. the order 5 inner automorphism of $\mathrm{E}_{8}$ ) in full, and simply summarize some salient data in the remaining cases.

In many instances the validity of the Theorem is related to delicate properties of the relevant groups in characteristic $p$. For example, when $\mathbf{G}$ is a split form of $\mathrm{Spin}_{8}$ and $\sigma$ is the pinned triality with fixed points $\mathrm{G}_{2}$, the $\sigma$-dual homomorphism $\mathrm{G}_{2} \rightarrow \mathrm{PSO}_{8}$ is not the "standard" eight-dimensional representation but (Proposition 11.5 and Lemma 11.4) its twist by the exceptional isogeny $\mathrm{G}_{2} \rightarrow \mathrm{G}_{2}$ that exists only in characteristic 3 . Also the Galois component of the $L$-group enters in an interesting way, especially when $p>2$ : roughly speaking, the action of ${ }^{L} \hat{\psi}$ on the "Galois component" of ${ }^{L} \hat{H}$ must exactly compensate the difference between half-sum shifts for $\mathbf{H}$ and $\mathbf{G}$ (a simple example is the inner automorphism of $\mathrm{G}_{2}$ of order 3, see Proposition 10.6).

If we relax the assumption that $\mathbf{G}$ is simply connected such an ${ }^{L} \hat{\psi}$ need not exist: a counterexample is given by taking $\sigma$ to be an inner automorphism of order 3 of $\mathbf{G}=$ $\mathrm{PGL}_{2}$. The difficulties arising here are related to the ambiguity of square roots in the Satake transform. A plausible solution is described in $\S 7.8$ : one can replace the $L$-group by a different extension $\hat{G} \rightarrow{ }^{c} \hat{G} \rightarrow \operatorname{Gal}(\bar{F} / F)$ but without the "pinned splitting" of ${ }^{L} \hat{G}$, with respect to which the Satake isomorphism can be formulated canonically (Theorem 7.9). It tempting to speculate then that there is always a $\sigma$-dual map ${ }^{c} \hat{H} \rightarrow{ }^{c} \hat{G}$. This variant ${ }^{c} \hat{G}$ is a subgroup of the " $C$-group" discussed in [9] and is closely related to the ideas of Deligne [16]. Since this story is somewhat orthogonal to our main goals we do not examine it further in this paper. It would also be interesting to relax the assumption that $\mathbf{H}$ is connected. In that case it appears that the First Main Theorem, suitably interpreted, remains valid, but we have not investigated if it has an $L$-group formulation.

1.3. Discussion. In the local setting, this correspondence is related to the "Brauer correspondence" of modular representation theory. In the global setting, the correspondence can be viewed as a kind of "mod $p$ Eisenstein series;" like Eisenstein series, many phenomena related to the Langlands program simplify but yet do not become trivial.

\footnotetext{
${ }^{1}$ In this case, $\mathbf{H}$ is automatically connected.

${ }^{2}$ The remaining cases could presumably be similarly treated, but the authors were left exhausted by the existing proof.
} 
Indeed, Eisenstein series are, in a sense, dual to the operation of restricting an automorphic form to the boundary. Here we observe that the symmetric space for $\mathbf{G}^{\sigma}$, embedded in the symmetric space for $\mathbf{G}$, behaves with respect to characteristic p homology like a kind of "interior boundary." A better-known example of this phenomenon is that "restriction to supersingular points" gives, for classical modular forms, a geometric construction of the Jacquet-Langlands correspondence modulo $p$.

The main technical tool to prove the "interior boundary" property is Smith theory, or $\mathbf{Z} / p$-equivariant localization. It is related to the prior paper [33] of the first-named author. If we call $Y$ (resp. $X$ ) the locally symmetric space for $\mathbf{G}^{\sigma}$ (resp. G) and put $\Gamma=\langle\sigma\rangle$, then the inclusion $Y \hookrightarrow X$ induces (almost) an isomorphism on equivariant cohomology $H_{\Gamma}^{*}(X) \rightarrow H_{\Gamma}^{*}(Y)$, and what remains is "just" to pass from equivariant cohomology to usual cohomology, and to understand Hecke actions.

Given a sufficiently good chain-level understanding of the $\sigma$-action on the cohomology of $X$, for instance a compatible triangulation of $X$, our method gives an explicit recipe for lifting automorphic forms on $Y$ to $X$. The recipe can be presented as a spectral sequence (see proof of Theorem 4.4). We do not expect it to degenerate and indeed the differentials seem to carry interesting information. It will be interesting to study this further.

One cannot be too optimistic about "lifting" the method to characteristic zero in any direct way. The proof uses special properties of the Frobenius at various points; in fact the homomorphism of dual groups mentioned above need not lift to characteristic zero, as in the example above, or the inner examples of [33]. Nonetheless the results here could be lifted to characteristic zero, using more machinery, in the following situation:

(i) There is a $\sigma$-dual homomorphism ${ }^{L} \hat{H} \rightarrow{ }^{L} \hat{G}$ and it lifts to characteristic zero,

(ii) $\mathrm{On} \mathbf{H}$ one may associate Galois representations to $\bmod p$ forms;

(iii) On $\mathbf{G}$ one has available modularity lifting theorems.

then one can in fact deduce the corresponding functoriality in characteristic zero (i.e., for cohomological automorphic forms) from the above Theorem. The recent work of Scholze and Calegari-Geraghty $[28,10]$ enlarges the list of possibilities where (ii) and (iii) apply. We do not pursue this here.

We note some related work. One inspiration for this paper was trying to understand the ideas behind the Glauberman correspondence [19]. In a Langlands setting the closest paper appears to be the recent work of Clozel [14]; it is closely related to the ideas of the current paper, specialized to the case of $\mathbf{G}$ a definite quaternion algebra over a totally real number field; it moreover makes intriguing use of this idea in the context of an infinite $p$-adic tower (see §1.4). The paper [33] of the first author, already mentioned, studies a similar story in the setting of the local geometric Satake correspondence, when $\sigma$ is inner. L. Clozel has pointed out that the arguments of $\S 8$ resemble some of the constructions in the theory of twisted endoscopy, as in [24], but in our case these constructions are on the dual side and in characteristic $p$. The paper of Kionke [23] applies the Smith inequalities to $p$-adic analytic towers of locally symmetric spaces for G. Finally we draw attention to the paper of Ash [1], which uses Smith theory to produce homology for $\mathrm{GL}_{n}$ over certain fields.

1.4. Open questions. We mention five interesting open questions:

(i) The ramified correspondence: we formulate a conjecture in $\S 6$ relating local functoriality to Tate cohomology. This is a problem solely in the representation theory of $p$-adic groups. N. Ronchetti has obtained some evidence for this, in the setting of cyclic base change for $\mathrm{GL}_{n}$ and supercuspidal representations. 
(ii) Behavior in a $p$-adic tower: In the case of a definite quaternion algebra, Clozel [14] formulates a theory of "automorphic forms over $\mathbf{Q}\left(\zeta_{p^{\infty}}\right)$." Can one make a similar theory for cohomological forms on an arbitrary group, using the ideas of the current paper?

(iii) Generalization of second main theorem: replacing the $L$-group by the $c$-group that we define in $\$ 7$, establish the existence of $\sigma$-dual homomorphisms in all cases that is, without any restriction that $\mathbf{G}$ be simply connected. (Even the situation when $\mathbf{H}$ is a torus would be of interest.)

(iv) Spectral sequences: Study more carefully the higher differentials in the spectral sequence of Theorem 4.4. In our context, these higher differentials cannot always be zero, and it would be interesting to understand their arithmetic importance.

(v) In general the locally symmetric space $Y$ for $\mathbf{G}^{\sigma}$ is only part, a union of connected components, of the full space of $\sigma$-fixed points of the locally symmetric space $X$ of $\mathbf{G}$. The other components appear to be locally symmetric spaces for different $F$-forms of $\mathbf{G}^{\sigma}$. Smith theory realizes their cohomology as a subquotient of the cohomology of $X$, but we have not investigated the compatibility with Hecke actions.

1.5. Plan of the paper. $\S 2$ summarizes some of our notations, and $\S 3$ some basic facts about Tate cohomology for cyclic groups.

$\S 4$ and $\$ 5$ describe the Brauer homomorphism and describe the proof of the first main theorem.

$\S 6$ describes the situation at ramified places. The results of this section are also used in the later parts of the paper.

$\S 7$ consists of "folklore results" on the Satake transform. We have stated and proved them here because we do not know of a reference with characteristic $p$ coefficients. However, the formulation of Theorem 7.9 may be of independent interest.

$\S 8$ computes the unramified Brauer homomorphism in terms of Satake parameters. Rather than compute directly, we deduce the result by applying the results of $\S 6$ to unramified representations.

$\S 9$ collects some preliminaries to the proof of our second main theorem. $\S 10$ proves it in many cases when $\sigma$ is an inner automorphism, $\S 11$ proves it in many cases including when $\sigma$ is a pinned automorphism, and $\S 12$ handles all remaining cases by direct computation.

1.6. Acknowledgements. We thank Raphael Rouquier and Zhiwei Yun for sharing their ideas about the Glauberman correspondence, and Brian Conrad and Mark Reeder for taking time to answer several questions about algebraic groups. Finally we thank Laurent Clozel for several helpful discussions as well as comments on the manuscript.

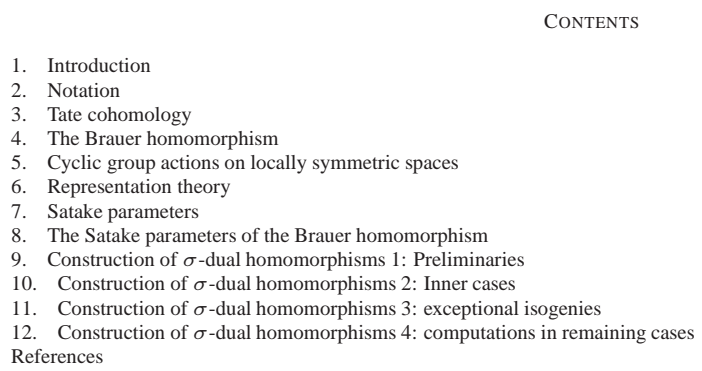




\section{NotAtion}

2.1. Notation used throughout. Let $p$ be a prime number and let $k$ be an algebraic closure of the field with $p$ elements. Let $\Lambda$ denote the ring of Witt vectors of $k$ (thus, $p$ is a uniformizer for $\Lambda$ ) and let $\Lambda\left[p^{-1}\right]$ denote the fraction field of $\Lambda$. For $x \in \Lambda$ let $\bar{x} \in k$ denote its reduction $\bmod p$.

The symbol $\sigma$ denotes a generator for a group of order $p$. We write $\langle\sigma\rangle$ for this group.

Let $F$ be a number field, $\Gamma_{F}$ its absolute Galois group, $\mathcal{O}_{F}$ its ring of integers, $\mathbf{A}$ its adele ring, $\mathbf{A}_{\mathrm{f}}$ its ring of finite adeles, and set $F_{\infty}=F \otimes_{\mathbf{Q}} \mathbf{R}$. If $v$ is a place of $F$ then $F_{v}$ denotes the completion of $F$ at $v$. By cyclo $: \Gamma_{F} \rightarrow \mathbf{F}_{p}^{*} \subset k^{*}$ we mean the cyclotomic character, i.e. the action of $\Gamma_{F}$ on $p$ th roots of unity in $F$.

If $W$ is a $k$-vector space we denote by $W^{(p)}$ the Frobenius-twist of $W$, i.e. the space with the same underlying vectors but scalar multiplication modified: if - is the scalar multiplication in $W$ then the scalar multiplication $*$ in $W^{(p)}$ is given by $\lambda * w=\lambda^{1 / p} \cdot w$; equivalently, $W^{(p)}=W \otimes{ }_{(k, \text { Frob })} k$, so that $w \lambda \otimes \mu=w \otimes \lambda^{p} \mu$ for $\lambda \in k$.

When we write homology $H_{*}$ or cohomology $H^{*}$ of a topological space, we will always understand the coefficients to be taken in $k$, unless otherwise specified.

If $X=\operatorname{Spec}(R)$ is an affine algebraic variety over $k$ and $G$ an algebraic group acting on $X$, we denote by $X / / G$ the geometric quotient, i.e. the spectrum of $R^{G}$.

2.2. Nonabelian cohomology. If $\sigma$ is an order $p$ automorphism of a group $M$, we let $H^{1}(\sigma ; M)$ denote the nonabelian cohomology of $\langle\sigma\rangle$ with coefficients in $M$, i.e. cocycles $j:\langle\sigma\rangle \rightarrow M$ modulo coboundaries. Elements of $H^{1}(\sigma ; M)$ may be equivalently regarded as elements $j(\sigma) . \sigma \subset M . \sigma \subset M \rtimes\langle\sigma\rangle$ modulo $M$-conjugacy, or elements $j(\sigma) \in M$ up to twisted conjugacy.

If $N \subset M$ is a $\sigma$-stable subgroup, then we have the "long exact sequence" of nonabelian cohomology [29, §§I.5.4-I.5.5]

$$
N^{\sigma} \hookrightarrow M^{\sigma} \rightarrow(M / N)^{\sigma} \rightarrow H^{1}(\sigma ; N) \rightarrow H^{1}(\sigma ; M)
$$

If $N$ is normal in $M$ and nilpotent of order prime to $p$, then $H^{1}(\sigma ; M) \rightarrow H^{1}(\sigma ; M / N)$ is a bijection.

2.3. Algebraic groups and level structures. Let $\mathbf{G}$ be a connected reductive algebraic group over $F$. If $v$ is a place of $F$, then $\mathbf{G}\left(F_{v}\right)$ is a locally compact topological group that we denote by $G_{v}$. If $v$ is a finite place, $K_{v}$ will denote an open compact subgroup of $G_{v}$. If $v$ is an archimedean place, $K_{v}$ denotes a maximal compact subgroup of $G_{v}$, and finally $K_{\infty}$ denotes a maximal compact subgroup of $G_{\infty}=\mathbf{G}\left(F_{\infty}\right)$.

By a level structure for $\mathbf{G}$, we mean an open compact subgroup $K \subset \mathbf{G}\left(\mathbf{A}_{\mathrm{f}}\right)$ of the form $\prod_{v} K_{v}$. Note for such a level structure $K_{v}$ is a "standard" maximal compact for almost all $v$ (i.e. is obtained by taking $\mathcal{O}_{v}$-points of an integral model of $\mathbf{G}$ over $\mathcal{O}_{F}$ ).

When a level $K$ is fixed then for $V$ a set of places of $F$, we denote by $G_{V}$ the restricted product $\prod_{v \in V}^{\prime} G_{v}=\left\{\left(g_{v}\right)_{v \in V} \mid g_{v} \in K_{v}\right.$ for all but finitely many $\left.v\right\}$. When $V$ is a set of finite places, we denote by $K_{V}$ the product $\prod_{w \in V} K_{w}$ and by $K^{(V)}$ the complementary subgroup

$$
K^{(V)}:=K_{\infty} \prod_{w \notin V} K_{w}
$$

where the product is taken over finite places $w$ not belonging to $V$. 
2.4. Canonical torus. If $F$ is any field, and $\mathbf{G}$ is connected and reductive over $F$, there is a canonical algebraic torus defined over $F$ attached to $\mathbf{G}$. We denote it by $\mathbf{T}_{G}^{\mathrm{can}}$. We will follow $[15, \S 1.1]$.

If $\mathbf{G}$ is quasisplit we may describe $\mathbf{T}_{G}^{c a n}$ as the $\operatorname{limit} \underset{\lim }{\mathbf{B}} / \mathrm{R}_{u}(\mathbf{B})$ over $F$-rational Borel subgroups of $\mathbf{G}$, of the quotient torus of the Borels. In general, we pass to an extension over which $\mathbf{G}$ is quasisplit, and then descend the torus thus constructed; then there may be no inclusion of $\mathbf{T}_{G}^{\mathrm{can}}$ into $\mathbf{G}$ defined over $F$.

Let $X^{*}$ denote the character lattice of $\mathbf{T}_{G}^{\mathrm{can}} \times_{F} \bar{F}$ and $X_{*}$ the dual lattice. Then $\left(X^{*}, X_{*}\right)$ supports a canonical based root datum $\Psi(\mathbf{G})$.

Automorphisms of $\bar{F} / F$ induce an action $\theta_{G}: \Gamma_{F} \rightarrow \operatorname{Aut}(\Psi(\mathbf{G})) \subset \operatorname{Aut}\left(X^{*}\right)$. The permutation representation of $\Gamma_{F}$ on simple roots determines an étale algebra over $F$, and if $\mathbf{G}$ is semisimple the $F$-rational points of $\mathbf{T}_{G}^{c a n}$ are naturally identified with the units in this algebra.

2.5. Dual groups and $L$-groups. By default, we will regard all dual groups as reductive algebraic groups over $k$, i.e. in characteristic $p$.

The dual root datum to $\Psi(\mathbf{G})$ determines a pinned reductive algebraic group over $k$, which we denote by $\hat{G}$. Recall that a "pinning" is data $\left(\hat{T}, \hat{B},\left\{X_{i}\right\}\right)$ where $\hat{T} \subset \hat{B} \subset$ $\hat{G}$ are a maximal torus and Borel subgroup of $\hat{G}$, and each $X_{i}$ is a nonzero vector in a simple root space of $\operatorname{Lie}(\hat{B})$. A pinning determines a splitting $\operatorname{Out}(\hat{G}) \rightarrow \operatorname{Aut}(\hat{G})$, and an identification $\operatorname{Out}(\hat{G}) \simeq \operatorname{Aut}(\Psi(\mathbf{G}))$.

In fact the dual group and its pinning can be constructed over the prime field $\mathbf{F}_{p}$. It follows that $\hat{G}, \hat{B}, \hat{T}$ can be equipped with Frobenius endomorphisms that are defined over $k$, which we will denote by Frob.

The construction $\Psi(\mathbf{G}) \rightarrow \Psi(\mathbf{G})^{\vee} \rightarrow\left(\hat{G}, \hat{B},\left\{X_{i}\right\}\right)$ is functorial, and one obtains a $\Gamma_{F}$ action $\Gamma_{F} \rightarrow \operatorname{Out}(\hat{G}) \subset \operatorname{Aut}(\hat{G})$. We let ${ }^{L} \hat{G}$ denote the semidirect product $\hat{G} \rtimes \Gamma_{F}$.

We regard $\hat{G}$ and ${ }^{L} \hat{G}$ as algebraic groups over $k$ (the latter with an infinite component group), and denote their groups of $k$-points by $\hat{G}(k)$ and ${ }^{L} \hat{G}(k)$.

If $\alpha$ is a root in $\Psi(\mathbf{G})$, it determines a coroot for $\hat{G}$. We will use the notation $\alpha_{*}$ for this coroot (although a couple of times we will abuse notation and drop the subscript). Similarly, if $\alpha^{\vee}$ is a coroot for $\Psi(\mathbf{G})$, then we use $\alpha_{*}^{\vee}$ for the associated root in $\hat{G}$.

Suppose $\mathbf{H}$ is another algebraic group with $L$-group ${ }^{L} \hat{H}$. As $k$ is algebraically closed and $\hat{H}, \hat{G}$ are scheme-theoretically reduced, any algebraic morphism ${ }^{L} \hat{H} \rightarrow{ }^{L} \hat{G}$ is determined by its induced morphism on $k$-points ${ }^{L} \hat{H}(k) \rightarrow{ }^{L} \hat{G}(k)$. For our purposes (Langlands functoriality), we may therefore usually ignore the difference between them. (Note however that, as $k$ has positive characteristic, the map ${ }^{L} \hat{H} \rightarrow{ }^{L} \hat{G}$ can have nontrivial fibers as a map of schemes and yet induce an injection, or even an isomorphism, ${ }^{L} \hat{H}(k) \rightarrow{ }^{L} \hat{G}(k)$.)

2.6. Local $L$-groups. In the construction of $\S 2.5$, we may replace $F$ by $F_{v}$ for any finite place $v$, producing a group $\hat{G} \rtimes \Gamma_{F_{v}}$. When $\mathbf{G}$ splits over an unramified extension of $F_{v}$, we work with the smaller group ${ }^{L} \hat{G}_{v}:=\hat{G} \rtimes\left\langle\operatorname{Frob}_{v}\right\rangle$. Here $\left\langle\operatorname{Frob}_{v}\right\rangle$ denotes the discrete infinite cyclic group that topologically generates the unramified quotient of $\Gamma_{F_{v}}$.

2.7. Parabolics and Levis in $\mathbf{G}$ and $\hat{G}$. A $\mathbf{G}(\bar{F})$-conjugacy class of parabolic subgroups $\mathbf{P} \subset \mathbf{G} \times_{F} \bar{F}$ distinguishes a subset $\Delta_{P}$ of the simple roots of $\Psi(\mathbf{G})$ : the set of $\alpha$ for which $-\alpha$ is a root of $\mathbf{P}$ (for a conjugation action of $\mathbf{T}^{\mathrm{can}}$ induced by an arbitrary Borel subgroup $\mathbf{B} \subset \mathbf{P}$ and splitting of $\mathbf{B} \rightarrow \mathbf{T}^{\mathrm{can}}$, all defined over $\bar{F}$ ). 
We define a corresponding Levi subgroup $\hat{L}$ of $\hat{G}$ - the subgroup generated by $\hat{T}$ and the coroot homomorphisms $\mathrm{SL}_{2} \rightarrow \hat{G}$ corresponding to $\alpha \in \Delta_{P}$.

Then the abelianization $\mathbf{L}^{\mathrm{ab}}$ of the quotient Levi $\mathbf{L}$ of $\mathbf{P}$ is dual, as a torus, to the center of $\hat{L}$ - with notations as above, the character group of $\mathbf{L}^{\mathrm{ab}}$ is identified with $X^{*}\left(\mathbf{T}^{\mathrm{can}}\right) /\left\langle\alpha^{\vee}\right.$ : $\left.\alpha \in \Delta_{P}\right\rangle$, whereas the co-character group of $Z(\hat{L})$ is identified with the orthogonal complement of $\left\{\alpha_{*}^{\vee}: \alpha \in \Delta_{P}\right\}$ in $X_{*}(\hat{T})$.

2.8. Frobenius maps. We will use the notation "Frob" for the Frobenius endomorphism of any $k$-group scheme equipped with a descent to $\mathbf{F}_{p}$. This applies, in particular, to any group of the form $\hat{G}$; the fixed point subgroup of Frob is exactly the discrete set of $\mathbf{F}_{p^{-}}$ points of $\hat{G}$.

2.9. Class field theory. We'll recall part of the Langlands correspondence for tori [25].

Let $\mathbf{T}$ be an algebraic torus over a number field $F$. We say $\mathbf{T}$ is unramified at $v$ if $T_{v}:=\mathbf{T}\left(F_{v}\right)$ splits over an unramified extension of $F_{v}$. A homomorphism $T_{v} \rightarrow k^{*}$ is unramified if it is trivial on the maximal compact subgroup of $T_{v}$. An idele class character $\mathbf{T}(F) \backslash \mathbf{T}\left(\mathbf{A}_{\mathrm{f}}\right) \rightarrow k^{*}$ is said to be unramified at $v$ if its restriction to $T_{v}$ is unramified.

Set ${ }^{L} \hat{T}=\hat{T} \rtimes \Gamma_{F}$ as in $\S 2.5$. When $\mathbf{T}$ is unramified at $v$ set ${ }^{L} \hat{T}_{v}=\hat{T} \rtimes\left\langle\right.$ Frob $\left._{v}\right\rangle$ as in §2.6. Let $A_{v}$ denote the $F_{v}$-points of the maximal split subtorus $\mathbf{A}_{v} \subset \mathbf{T}$. (We leave in the subscript $v$ because this depends on the place $v$ ). Then when $\mathbf{T}$ is unramified at $v$ restriction gives an isomorphism (see $[4, \S 9.5])$ :

$$
\text { \{unramified characters of } \left.\left.T_{v}\right\} \stackrel{\sim}{\rightarrow} \text { unramified characters of } A_{v}\right\}
$$

There is also a natural surjective homomorphism $\hat{T} \rightarrow \hat{A}_{v}$ that identifies $\hat{A}_{v}$ with the Frob $_{v}$-coinvariants of $\hat{T}$. Therefore, to an unramified character of $T_{v}$ is associated an element of the coinvariants $\hat{T}_{\mathrm{Frob}_{v}}$; put another way, this gives a natural bijection between unramified characters of $T_{v}$ and conjugacy classes of splittings $\left\langle\right.$ Frob $\left._{v}\right\rangle \rightarrow{ }^{L} \hat{T}_{v}$. The element of $\hat{T}_{\mathrm{Frob}_{v}}$ associated to an unramified character $\chi$ will be called the "Langlands parameter" of $\chi$.

We can describe this more directly: Since $X^{*}(\mathbf{T})^{\operatorname{Frob}_{v}} \rightarrow X^{*}(\mathbf{A})$ is an injection with finite cokernel, and $k^{*}$ is divisible, we obtain a surjection $X^{*}(\mathbf{T})^{\operatorname{Frob}_{v}} \otimes k^{*} \rightarrow X^{*}(\mathbf{A}) \otimes$ $k^{*}$. Thus each element of $X^{*}(\mathbf{T})^{\mathrm{Frob}_{v}} \otimes k^{*}$ gives an unramified character $\chi$, and every unramified character $\chi$ arises thus, although possibly not uniquely. Explicitly, if $\alpha \in$ $X^{*}(\mathbf{T})^{\mathrm{Frob}_{v}}$ and $\lambda \in k^{*}$, the unramified character associated to $\alpha \otimes \lambda$ is given by $t \in$ $T_{v} \mapsto \lambda^{v(\alpha(t))}$.

One obtains the parameter of $\chi$ via the maps

$$
X^{*}(\mathbf{T}) \otimes k^{*}=X_{*}(\hat{T}) \otimes k^{*}=\hat{T}(k)
$$

(i.e., the character $\chi$ is parameterized by the splitting $\operatorname{Frob}_{v} \mapsto t_{\chi} \operatorname{Frob}_{v}$, where $t_{\chi} \in \hat{T}(k)$ is the element thus produced).

2.10. Hecke algebras. Let $G$ be a locally compact, totally disconnected group. If $S$ is a discrete set with a continuous left $G$-action and compact stabilizers, let $\operatorname{Fun}_{G}(S \times S)$ (pronounced "funguses") denote the set of $k$-valued functions on $S \times S$ that are invariant for the diagonal action of $G$, and whose support is a union of finitely many $G$-orbits. $\operatorname{Fun}_{G}(S \times S)$ has an algebra structure with multiplication given by

$$
\left(h_{1} * h_{2}\right)(x, z)=\sum_{y \in S} h_{1}(x, y) h_{2}(y, z)
$$


If $k[S]$ denotes the vector space spanned by $S$, there is a left action of $\operatorname{Fun}_{G}(S \times S)$ on $k[S]$ given by

$$
h * s=\sum_{t \in S} h(s, t) t
$$

If $S$ has finitely many $G$-orbits, $\operatorname{Fun}_{G}(S \times S)$ has a two-sided unit and the action on $k[S]$ identifies $\operatorname{Fun}_{G}(S \times S)$ with the ring of $G$-endomorphisms of $k[S]$.

The standard example is when $K \subset G$ is an open compact subgroup and $S=G / K$. In that case, $\operatorname{Fun}_{G}(G / K \times G / K)$ can be identified with finitely supported functions on the double coset space $K \backslash G / K$, via $h(K, g K)=h(K g K)$. We abbreviate this case by $\mathscr{H}(G, K)$. We will also use the notation $\mathscr{H}\left(G, K ; \mathbf{F}_{q}\right)$ for that subalgebra of $\mathscr{H}(G, K)$ consisting of functions valued in $\mathbf{F}_{q} \subset k$.

The theories of left- and right- $\mathscr{H}(G, K)$-modules are equivalent via the anti-involution $K g K \leftrightarrow K g^{-1} K$; nevertheless we wish to record some explicit formulas for these actions with some attention paid to the difference between left and right:

The identification $V^{K}=\operatorname{Hom}_{G}(k[G / K], V)$ gives the $K$-invariants of a left $G$-module the structure of a right $\mathscr{H}(G, K)$-module. When $V$ is a left $G$-module, an explicit formula for this action is

$$
v * h=\sum_{g K \in G / K} g^{-1} v h(K, g K)
$$

When $X$ is a set with a right $G$-action, the $k$-vector space $k[X]$ spanned by $X$ carries a left $G$-module structure extending linearly $g \cdot x=x g^{-1}$. Then (2.10.2) specializes to the following right $\mathscr{H}(G, K)$-action on $k[X / K] \simeq k[X]^{K}$ :

$$
x K * h=\sum_{g K \in G / K} x g K h(K, g K) \quad \text { for } x K \in X / K
$$

\subsection{Hecke actions on homology and cohomology. Suppose that:}

(i) $G$, as in $\$ 2.10$, is a locally compact, totally disconnected group, and $K \subset G$ an open compact subgroup.

(ii) $X$ is a locally compact Hausdorff topological space with continuous right $G$ action, such that the restriction of this action to $K$ is free and proper;

Note that the assumptions force $K$ to be profinite. Let $K_{i} \subset K$ be a collection of open normal subgroups with $\bigcap_{i} K_{i}=\{e\}$; then the natural map

$$
\pi: X \rightarrow \lim _{\longleftarrow} X / K_{n}
$$

is a homeomorphism. In fact, it is easily verified to be a continuous bijection. Now, we need to check that the image of any closed set $Z \subset X$ is also closed in $\lim X / K_{n}$. Choose $y \notin \pi(Z)$. We want an open set containing $y$ and disjoint from $\pi(\overleftarrow{Z})$. We may find a compact neighbourhood $A$ of $y$ in $X / K$ with $\pi^{-1} A$ also compact. Then $\pi(Z) \cap A=$ $\pi\left(Z \cap \pi^{-1}(A)\right)$ is compact inside $\lim X / K_{n}$. Thus there is an open set $N \ni y$ that's disjoint from $\pi(Z) \cap A$, and then $A^{\circ} \cap N$ is the required open set.

Then there is a right action of $\mathscr{H}(G, K)$ on the $k$-homology of $X / K$ and a left action on the $k$-cohomology of $X / K$. We give an explicit construction of it:

The set of singular $m$-simplices $\operatorname{Hom}\left(\Delta^{m}, X\right)$ has a right $G$-action. Since the $K$-action is free on $X$ it is free also on $\operatorname{Hom}\left(\Delta^{m}, X\right)$ and the quotient is naturally identified with the set of singular $m$-simplices in $X / K$ : Each map $X / K_{n} \rightarrow X / K$ is a covering space 
and by (2.11.1) each $m$-simplex in $X / K$ lifts to $X$ uniquely up to $\lim _{\longleftarrow} K / K_{n}=K$. Thus, we get a right action of $\mathscr{H}(G, K)$ on

$$
C_{m}(X / K)=k\left[\operatorname{Hom}\left(\Delta^{m}, X\right) / K\right]=k\left[\operatorname{Hom}\left(\Delta^{m}, X\right)\right]^{K}
$$

for each $m$ by applying the discussion around (2.10.3) with $S=\operatorname{Hom}\left(\Delta^{m}, X\right)$. As the face maps are maps of $\mathscr{H}(G, K)$-modules, the action descends to a right action of $\mathscr{H}(G, K)$ on $H_{m}(X / K ; k)$. The identification of $H^{m}(X / K)$ with the dual vector space to $H_{m}(X / K)$ gives it a left $\mathscr{H}(G, K)$-action.

2.12. Borel-de Siebenthal theory. The section title refers to the general structure theory for semisimple subgroups of $\mathbf{G}$ of the same rank as $\mathbf{G}$. In characteristic 0 , such subgroups are the fixed points of inner automorphisms of $\mathbf{G}$. We will recall that aspect of the theory that is important for us, when the inner automorphism has prime order $p$.

Let $\mathbf{G}$ be a simply-connected algebraic group over $\bar{F}$, and let $\mathbf{B}_{G} \supset \mathbf{T}$ be a maximal torus and Borel in $\mathbf{G}$, and let $\Delta(\mathbf{G})=\left\{\alpha_{i}\right\}$ denote the corresponding set of simple roots. Let $\alpha_{0}$ be the highest root. Let $\omega_{i}^{\vee}: \mathbb{G}_{m} \rightarrow \mathbf{T} / Z(\mathbf{G})$ denote the $i$ th fundamental weight, so that the coefficient of $\alpha_{i}$ in $\alpha_{0}$ is $\left\langle\omega_{i}^{\vee}, \alpha_{0}\right\rangle$.

Theorem. Suppose $\left\langle\omega_{i}^{\vee}, \alpha_{0}\right\rangle=p$. Then there is a unique connected and semisimple subgroup $\mathbf{H} \subset \mathbf{G}$, containing $\mathbf{T}$, with the following properties:

(1) $-\alpha_{0}$ and $\alpha_{j}$ are roots of $\mathbf{T}$ on $\mathbf{H}$. Meanwhile, $\alpha_{i}$ is not a root of $\mathbf{H}$.

(2) The set $\Delta(\mathbf{H})=\left\{-\alpha_{0}\right\} \cup\left\{\alpha_{j}\right\}_{j \neq i}$ is a system of simple roots for $\mathbf{H}$, which can be realized as a subset of the extended Dynkin diagram $\tilde{\Delta}(\mathbf{G})$ by removing vertex $i$.

Recall the method of proof ([5], [27, §2]): One sees that if $\mathbf{H}$ exists, the quotient $Z(\mathbf{H}) / Z(\mathbf{G})$ must have an element of order $p$ represented by the image under $\omega_{i}^{\vee}$ of a primitive $p$ th root of unity $\zeta$. One then constructs $\mathbf{H}$ as the centralizer of a representative $s \in \mathbf{T}$ for $\omega_{i}^{\vee}(\zeta)$.

The automorphism group of the extended Dynkin diagram of $\widetilde{\Delta}(\mathbf{G})$ permutes the $\omega_{i}^{\vee}$ with $\left\langle\omega_{i}^{\vee}, \alpha_{0}\right\rangle=p$. If $\omega_{i}^{\vee}$ and $\omega_{j}^{\vee}$ are in the same orbit, the corresponding subgroups $\mathbf{H}$ are $\mathbf{G}(\bar{F})$-conjugate. This provides a complete list of $\mathbf{G}(\bar{F})$-conjugacy classes of inner automorphisms of order $p$ whose fixed points are semisimple.

2.13. Endoscopic subgroups. If $\hat{G}$ is an algebraic group of low characteristic (for us, the dual group of a simply connected group $\mathbf{G}$ ), there are some exceptional semisimple subgroups of $\hat{G}$, of full rank, not captured by Borel-de Siebenthal. Because they arise in duality with the centralizers of $\mathbf{G}$ it is tempting to call such subgroups "endoscopic subgroups." ${ }^{3}$ In this section we recall a parametrization of these subgroups parallel to that of $\S 2.12$.

Let $\hat{T}_{G} \subset \hat{B}_{G} \subset \hat{G}$ be the pinned maximal torus and Borel subgroup in $\hat{G}$ over $k$, as in $\S 2.5$. Let $\Delta(\hat{G})=\left\{\alpha_{i, *}^{\vee}\right\}$ denote the corresponding set of simple roots, and let $\alpha_{0, *}^{\vee}$ denote the highest short root. (We are following the notation for roots of dual groups discussed in §2.5). Let $\alpha_{i, *}$ and $\alpha_{0, *}$ denote the associated coroots to the roots $\alpha_{i, *}^{\vee}$ and $\alpha_{0, *}^{\vee}$ - thus $\alpha_{0, *}^{\vee}$ is the highest coroot of $\hat{G}$. Let $\left\langle\omega_{i, *}^{\vee}, \alpha_{0, *}\right\rangle$ denote the coefficient of $\alpha_{i, *}$ in $\alpha_{0, *}$.

Theorem. Suppose $\left\langle\omega_{i, *}^{\vee}, \alpha_{0, *}\right\rangle=p$. Then there is a unique connected and semisimple subgroup $\hat{H}_{0}$ of $\hat{G}$, containing $\hat{T}_{G}$, with the following properties:

\footnotetext{
${ }^{3}$ But note that in our story these groups appear on the Galois side. In the theory of endoscopy they appear on the automorphic side, where they are not always subgroups.
} 
(1) $-\alpha_{0, *}^{\vee}$ and $\alpha_{j, *}^{\vee}$ for $j \neq i$ are roots of $\hat{T}_{G}$ on $\hat{H}_{0}$. Meanwhile $\alpha_{i, *}^{\vee}$ is not a root of $\hat{H}_{0}$.

(2) The set $\Delta\left(\hat{H}_{0}\right):=\left\{-\alpha_{0, *}^{\vee}\right\} \cup\left\{\alpha_{j, *}^{\vee}\right\}_{j \neq i}$ make a system of simple roots for $\hat{H}_{0}$, which can be realized as a subset of the extended Dynkin diagram of $\hat{G}$ by removing $\alpha_{i, *}^{\vee}$ from $\widetilde{\Delta}(\hat{G})$.

A case-by-case proof is given in [33, Theorem 3.3] but it does not give as much precision on the root systems, so we sketch a proof now:

Proof. To specify a smooth subgroup scheme of $\hat{G}$ or G we must specify a "quasi-closed" subset $S$ of roots for $\mathbf{G}$ or $\hat{G}$ - see [6, §3] but also [30, Corollary 5] for a reference in a form more convenient to us. Let us for simplicity suppose $\mathbf{G}$ does not have type $\mathrm{G}_{2}$, though that case can be verified either directly or by a similar argument. If $\mathbf{G}$ does not have type $\mathrm{G}_{2}$, then "quasi-closed" means (loc. cit.) the following: If $\alpha, \beta \in S$ and $i, j>0$ are such that $i \alpha+j \beta$ is a root for $G$, and furthermore the commutator coefficient $N_{\alpha \beta, i j}$ (in the notation of [30]) is not divisible by $p$, then in fact $i \alpha+j \beta \in S$.

In view of the computations of [34] we may rephrase this condition for symmetric $S$ (i.e. $S=-S$ ) as follows: if $\alpha$ and $\beta$ are in $S$ and $\Psi$ is the root system they span, then either $\Psi \cap S=\Psi$, or $\Psi \cap S$ is the set of long roots of $\Psi$, or else $\Psi \cap S$ is the set of short roots of $\Psi$. The last possibility occurs only when $p=2$.

To prove the Theorem, we prove that if $S$ is the quasi-closed set of roots that arise from $\mathbf{H}$ as in Theorem 2.12, the set $\left\{\alpha_{*}^{\vee}: \alpha \in S\right\}$ is also quasi-closed. As $\mathbf{H}$ is reductive, $S$ is symmetric, and according to the criterion for symmetric $S$ this is automatic if $\mathbf{G}$ is simply laced, or $p=2$. The only remaining cases are $\mathrm{F}_{4}$ and $\mathrm{G}_{2}$ with $p=3$, which can be verified by hand.

A more uniform "Tannakian" approach is proposed in loc. cit., but to carry out the proposal requires a better understanding of Smith theory for perverse sheaves [33, Conjecture 4.18].

\section{TATE COHOMOLOGY}

3.1. Definition of Tate cohomology. Let $M$ be an abelian group with an action of the cyclic group $\langle\sigma\rangle$ of order $p$ generated by $\sigma$. Set $\mathrm{T}^{0}(M):=\operatorname{ker}(1-\sigma) / \operatorname{Im}(N)$ and $\mathrm{T}^{1}(M):=\operatorname{ker}(N) / \operatorname{Im}(1-\sigma)$, where $N=1+\sigma+\cdots+\sigma^{p-1}$ is the "norm." In other words, $\mathrm{T}^{i}(M)$ is the cohomology of the 2-periodic chain complex whose differentials alternate between $1-\sigma$ and $N$. Because of this each short exact sequence of $\sigma$-modules induces a long exact sequence

$$
\mathrm{T}^{0}\left(M^{\prime}\right) \rightarrow \mathrm{T}^{0}(M) \rightarrow \mathrm{T}^{0}\left(M^{\prime \prime}\right) \rightarrow \mathrm{T}^{1}\left(M^{\prime}\right) \rightarrow \mathrm{T}^{1}(M) \rightarrow \mathrm{T}^{1}\left(M^{\prime \prime}\right) \rightarrow \mathrm{T}^{0}\left(M^{\prime}\right)
$$

3.2. Tate on smooth functions on $\ell$-spaces. If $X$ is a Hausdorff, locally compact, totally disconnected space (an " $\ell$-space", in the terminology of [2]) write $C_{c}^{\infty}(X ; \Lambda)$ or $C_{c}^{\infty}(X ; k)$ for the space of $\Lambda$ - or $k$-valued functions on $X$ that are locally constant ("smooth") and compactly supported. If $\sigma$ acts continuously on $X$ then we may form $\mathrm{T}^{i}\left(C_{c}^{\infty}(X ; ?)\right)$. These groups can be computed in terms of $X^{\sigma}$, as follows:

(1) For $i=0$ or 1 , restricting to fixed points descends to an isomorphism

$$
\mathrm{T}^{i}\left(C_{c}^{\infty}(X ; k)\right) \stackrel{\sim}{\rightarrow} C_{c}^{\infty}\left(X^{\sigma} ; k\right)
$$

(2) The map

$$
\mathrm{T}^{0}\left(C_{c}^{\infty}(X ; \Lambda)\right) \rightarrow C_{c}^{\infty}\left(X^{\sigma} ; k\right)
$$


give by restricting to fixed points and reducing $\bmod p$ (in either order) is an isomorphism, while $\mathrm{T}^{1}\left(C_{c}^{\infty}(X ; \Lambda)\right)=0$.

We are going to prove a more general result in the Proposition below.

3.3. Tate on sheaves on $\ell$-spaces. Let $X$ be as in $\S 3.2$, and let $\mathscr{F}$ be a sheaf of $k$-or $\Lambda$-modules on $X$. Write $\Gamma_{c}(X ; \mathscr{F})$ for the space of compactly supported sections of $\mathscr{F}$. For instance, if $\mathscr{F}$ is the constant sheaf with stalk $k$ or $\Lambda$ then $\Gamma_{c}(X ; \mathscr{F})=C_{c}^{\infty}(X ; k)$ or $C_{c}^{\infty}(X ; \Lambda)$. The assignment $\mathscr{F} \mapsto \Gamma_{c}(X, \mathscr{F})$ is a covariant exact functor $[3, \S 1.3]$.

If $\sigma$ acts on $X$ and $\mathscr{F}$ is $\sigma$-equivariant then $\sigma$ can be regarded as a map of sheaves $\left.\left.\mathscr{F}\right|_{X^{\sigma}} \stackrel{\sigma}{\rightarrow} \mathscr{F}\right|_{X^{\sigma}}$ and we may define

$$
\begin{array}{ll}
\mathrm{T}^{0}\left(\left.\mathscr{F}\right|_{X^{\sigma}}\right)=\operatorname{ker}(1-\sigma) / \operatorname{Im}(N) & \left(\text { a sheaf on } X^{\sigma}\right) \\
\mathrm{T}^{1}\left(\left.\mathscr{F}\right|_{X^{\sigma}}\right)=\operatorname{ker}(N) / \operatorname{Im}(1-\sigma) & \left(\text { a sheaf on } X^{\sigma}\right)
\end{array}
$$

A compactly supported section of $\mathscr{F}$ can be restricted to a compactly supported section of $\left.\mathscr{F}\right|_{X^{\sigma}}$. This map preserves the $\sigma$-actions inducing a map

$$
\mathrm{T}^{i}\left(\Gamma_{c}(X ; \mathscr{F})\right) \rightarrow \Gamma_{c}\left(X^{\sigma} ; \mathrm{T}^{i}(\mathscr{F})\right)
$$

Proposition. The map (3.3.1) is an isomorphism.

In the proof we'll use "existence of fundamental domains:" if $\sigma$ acts freely on a compact $\ell$-space $X$, there is a fundamental domain, i.e. a closed and open subset $F \subset X$ so that $X$ is the disjoint union of $\sigma^{i} F$ for $0 \leq i \leq p-1$. Indeed, take a cover of $X$ by finitely many closed-and-open sets $U_{i}$ so that $\sigma\left(U_{i}\right) \cap U_{i}=\emptyset$, take the algebra of sets generated (under intersection and complement) by the $U_{i}$ and their images under $\langle\sigma\rangle$. The minimal nonempty elements of that algebra give a finite compatible partition of $X$, each block of the partition being disjoint from its $\sigma$-image. The desired domain now follows by taking representatives for the $\sigma$-orbits on the blocks.

Proof. There is a short exact sequence (cf. $[2,1.16]$ )

$$
0 \rightarrow \mathscr{F}^{\prime} \rightarrow \mathscr{F} \rightarrow \mathscr{F}^{\prime \prime} \rightarrow 0
$$

of $\sigma$-equivariant sheaves where $\mathscr{F}^{\prime \prime}$ is supported on $X^{\sigma}$ and the stalks of $\mathscr{F}^{\prime}$ vanishes along $X^{\sigma}$. It suffices to treat the cases $\mathscr{F}=\mathscr{F}^{\prime \prime}$ and $\mathscr{F}=\mathscr{F}^{\prime}$. For $\mathscr{F}^{\prime \prime}$, the Proposition follows from the exactness of $\Gamma_{c}$ applied to the 2-periodic chain complex defining $\mathrm{T}^{*}$. So it is enough to check vanishing of $\mathrm{T}^{i}\left(\Gamma_{c}\left(U ; \mathscr{F}^{\prime}\right)\right)$ when $U^{\sigma}$ is empty.

We check for $\mathrm{T}^{0}$, the other case being similar. If $f \in \Gamma_{c}\left(U, \mathscr{F}^{\prime}\right)$ is $\sigma$-invariant, choose a $\sigma$-invariant compact $U^{\prime} \subset U$ containing the support of $f$, take a fundamental domain $F^{\prime} \subset U^{\prime}$ for the $\sigma$-action, and note $f=\mathrm{N}\left(f^{\prime}\right)$, where $f^{\prime}$ is a section which agrees with $f$ on $F^{\prime}$ and is zero off $F^{\prime}$.

3.4. Tate on rings. There is an algebraic relative of $\$ 3.2$ :

If $A$ is any commutative unital $k$-algebra with a $\sigma$-action, set $\bar{A}=\mathrm{T}^{0} A=A^{\sigma} / N A$. It has a ring structure because $N \cdot A$ is an ideal in $A^{\sigma}$. Then we have a bijection

$$
\operatorname{Hom}(A, k)^{\sigma} \stackrel{\sim}{\longrightarrow} \operatorname{Hom}(\bar{A}, k) .
$$

defined by restriction of characters.

Proof. For short, let us say an "extension" of a character $\psi: \bar{A} \rightarrow k$ is a character $\chi:$ $A \rightarrow k$ such that $\chi$, when restricted to $A^{\sigma}$, factors as $A^{\sigma} \rightarrow \bar{A} \stackrel{\psi}{\rightarrow} k$.

There is a ring homomorphism $A \rightarrow \bar{A}$ given by $a \mapsto\left(a a^{\sigma} \ldots a^{\sigma^{p-1}}\right)$. When we restrict this to $A^{\sigma}$, it gives the $p$ th power of the tautological map $A^{\sigma} \rightarrow \bar{A}$. 
Thus given a character $\chi$ on $\bar{A}$ the formula

$$
\tilde{\chi}(a)=\chi\left(a a^{\sigma} \ldots a^{\sigma^{p-1}}\right)^{1 / p}
$$

defines an extension to $A$. This is the unique extension of $\chi$ to $A$ : it is clearly the only possible $\sigma$-fixed extension, and in fact any extension of $\chi$ to $A$ must be $\sigma$-fixed. To see this, note that as $\chi$ is trivial on $N A$ we must have $\sum_{i=0}^{p-1} \tilde{\chi}^{\sigma^{i}}=0$ for any extension $\tilde{\chi}$. By linear independence of characters $\left[8, \mathrm{Ch} . \mathrm{V} \S 6.1\right.$, Theorem 1], it follows that $\tilde{\chi}^{\sigma^{i}}=\tilde{\chi}$, i.e. $\tilde{\chi}_{1} \in \operatorname{Hom}(A, k)^{\sigma}$.

The proof shows, more generally, that given any commutative integral domain $B$, any homomorphism $\chi: \bar{A} \rightarrow B$ has the property that $\chi^{p}$ extends uniquely to $A$ (same argument, replacing $B$ by its quotient field to invoke the linear independence of characters).

\section{THE BRAUER HOMOMORPHISM}

4.1. $\sigma$-actions, $\sigma$-plain subgroups. Let $G$ be a locally compact, totally disconnected group, and $K \subset G$ an open compact subgroup. Suppose $\sigma$ acts on $G$ with $\sigma(K)=K$ and $\sigma^{p}=1$. Write $G^{\sigma}$ and $K^{\sigma}$ for the fixed subgroups.

If $X$ is a right $G$-space on which $K$-acts freely, then $\mathscr{H}(G, K)$ acts on the chains and cochains of $X / K$ and $\mathscr{H}\left(G^{\sigma}, K^{\sigma}\right)$ acts on the chains and cochains of $X^{\sigma} / K^{\sigma}$. Note the difference between $(X / K)^{\sigma}$ and its subspace $X^{\sigma} / K^{\sigma}$ — the former usually does not carry a $\mathscr{H}\left(G^{\sigma}, K^{\sigma}\right)$-action.

We wish to relate the Hecke modules $H^{*}(X / K)$ and $H^{*}\left(X^{\sigma} / K^{\sigma}\right)$. The relationship becomes much simpler under a technical hypothesis on $K$. We say that $K \subset G$ is $\sigma$-plain if both of the following conditions hold:

(a) the inclusion

$$
G^{\sigma} / K^{\sigma} \hookrightarrow(G / K)^{\sigma}: g K^{\sigma} \mapsto g K
$$

is a bijection, or equivalently if $G^{\sigma}$ acts on $(G / K)^{\sigma}$ with a single orbit, or equivalently if $H^{1}(\sigma, K) \rightarrow H^{1}(\sigma, G)$ has trivial fiber above the trivial class.

(b) $K$ is virtually prime-to- $p$, i.e. there is a finite index subgroup $K^{\prime} \subset K$ which is a projective limit of prime-to- $p$ finite groups. In particular $H^{1}(\sigma, K)$ is finite (\$2.2).

4.2. The Brauer homomorphism. Suppose $\sigma$ acts on $G$ and $K$ as in $\S 4.1$. The algebra $\mathscr{H}(G, K)$ has an action of $\sigma$ (i.e. $h^{\sigma}\left(x^{\sigma}\right)=h(x)$ for $\left.x \in G / K \times G / K\right)$. Write $\mathscr{H}(G, K)^{\sigma}$ for the $\sigma$-invariant part of $\mathscr{H}(G, K)$. If $K$ is $\sigma$-plain, then the Brauer homomorphism is the map

$$
\mathrm{Br}: \mathscr{H}(G, K)^{\sigma} \rightarrow \mathscr{H}\left(G^{\sigma}, K^{\sigma}\right)
$$

just given by restricting $h$ from $G / K \times G / K$ to $\left((G / K)^{\sigma} \times(G / K)^{\sigma}\right)=\left(G^{\sigma} / K^{\sigma}\right) \times$ $G^{\sigma} / K^{\sigma}$. Since the summands of (2.10.1) are invariant under the action of $\sigma$ on $G / K$, and $k$ has characteristic $p$, the Brauer map is an algebra homomorphism. A similar construction is called the "Brauer homomorphism" in the modular representation theory of finite groups, and we call it by the same name here.

Set $N=1+\sigma+\sigma^{2}+\cdots+\sigma^{p-1}$, i.e. $N$ is the "norm element" in the group ring $k[\sigma]$. Then $N \cdot(1-\sigma)=(1-\sigma) \cdot N=0$. If $\sigma$ acts on a set $S$, and we thereby regard $k[S]$ as a $k[\sigma]$-module, there are canonical identifications

$$
\operatorname{ker}(1-\sigma) / \operatorname{Im}(N)=k\left[S^{\sigma}\right], \quad \operatorname{ker}(N) / \operatorname{Im}(1-\sigma)=k\left[S^{\sigma}\right]
$$

Note these are the groups $\mathrm{T}^{i}$ from $\S 3.1$. The identification on the left sends $s \in S^{\sigma}$ to $s+\operatorname{Im}(N)$ and on the right it sends $s \in S^{\sigma}$ to $s+\operatorname{Im}(1-\sigma)$. The Brauer homomorphism 
is compatible with these identifications in a sense we now describe (see also (6.2.1) for a more general statement):

Suppose that $\tilde{S}$ is a right $G$-set with compatible $\sigma$-action such that $K$ acts freely, $S=$ $\widetilde{S} / K$ and $h \in \mathscr{H}(G, K)^{\sigma}$. Then the map $i: \tilde{S}^{\sigma} / K^{\sigma} \rightarrow S^{\sigma}$ is injective as the $K$ action is free. Moreover, $\mathscr{H}(G, K)^{\sigma}$ acts on $k\left[S^{\sigma}\right] \simeq \mathrm{T}^{0} k[S]$ by means of (4.2.1), i.e. each $h \in \mathscr{H}$ acts via $\mathrm{T}^{0} h$.

We claim that, in fact, $k\left[\tilde{S}^{\sigma} / K^{\sigma}\right]$ is a $\mathscr{H}(G, K)^{\sigma}$-direct summand of $k\left[S^{\sigma}\right]$, and (4.2.2)

The action of $\mathrm{T}^{0} h$ for $h \in \mathscr{H}(G, K)^{\sigma}$ on $k\left[\tilde{S}^{\sigma} / K^{\sigma}\right]$ coincides with $\operatorname{Br}(h) \in \mathscr{H}\left(G^{\sigma}, K^{\sigma}\right)$.

Moreover, the identical statement holds also for $\mathrm{T}^{1}$.

It is enough to show the same statement with "direct summand" replaced by "submodule"; then one notes that the natural $k$-valued bilinear pairing on $k\left[S^{\sigma}\right]$ — given by $\left\langle\sum a_{s} s, \sum b_{s} s\right\rangle=\sum a_{s} b_{s}$ - has the property that $\left\langle a * \mathrm{~T}^{0} h, b\right\rangle=\left\langle a, b * \mathrm{~T}^{0} h^{\prime}\right\rangle$ where $h \mapsto h^{\prime}$ is the antiinvolution of the Hecke algebra sending $K g K$ to $K g^{-1} K$. Moreover, this bilinear pairing is nondegenerate on $k\left[\tilde{S}^{\sigma} / K^{\sigma}\right]$. That shows that $k\left[\tilde{S}^{\sigma} / K^{\sigma}\right]$ is actually a summand.

Now to check (4.2.2): For $\tilde{s} \in \tilde{S}^{\sigma}$ we have $\tilde{s} K^{\sigma} * \operatorname{Br}(h)=\sum_{g \in G^{\sigma} / K^{\sigma}} \tilde{s} g K^{\sigma} h(K, g K)$ and $\tilde{s} K * h=\sum_{g \in G / K} \tilde{s} g K h(K, g K)$. Considered in $k[S]^{\sigma}$, these elements differ by

$$
\sum_{g \in G / K-\left(G^{\sigma} / K^{\sigma}\right)} \tilde{s} g K h(K, g K)
$$

and our assumption that $K$ is $\sigma$-plain means that $\sigma$ acts freely on $G / K-\left(G^{\sigma} / K^{\sigma}\right)$; in particular, the element above belongs to the image of $N$ from (4.2.1).

4.3. Normalized Brauer homomorphism. Suppose $\mathscr{H}(G, K)$ and $\mathscr{H}\left(G^{\sigma}, K^{\sigma}\right)$ are commutative integral domains. Then according to $\S 3.4$, the $p$ th power of $\mathrm{Br}$ extends uniquely to a homomorphism

$$
\widetilde{\mathrm{Br}}: \mathscr{H}(G, K) \rightarrow \mathscr{H}\left(G^{\sigma}, K^{\sigma}\right) .
$$

This map is not $k$-linear but rather Frobenius semilinear. However, we may twist it to be linear: $\mathscr{H}(G, K)$ has an $\mathbf{F}_{p}$-structure, i.e.

$$
\mathscr{H}(G, K)=\operatorname{Fun}_{G}\left(G / K \times G / K ; \mathbf{F}_{p}\right) \otimes_{\mathbf{F}_{p}} k
$$

The normalized Brauer homomorphism, which we denote with a lower case "b," is the unique $k$-linear homomorphism

$$
\text { br : } \mathscr{H}(G, K) \longrightarrow \mathscr{H}\left(G^{\sigma}, K^{\sigma}\right),
$$

that agrees with $\widetilde{\mathrm{Br}}$ on $\operatorname{Fun}_{G}\left(G / K \times G / K ; \mathbf{F}_{p}\right)$. An explicit formula for br is given by

$$
\operatorname{br}(h)\left(K^{\sigma}, g K^{\sigma}\right)=((h * \cdots \cdot \cdots h)(K, g K))^{\frac{1}{p}} .
$$

4.4. Theorem. Let $G, K, X$ be as in $\$ 2.11$. Suppose that $\sigma$ acts compatibly on $G, K, X$, so that $G^{\sigma}, K^{\sigma}, X^{\sigma}$ also satisfy the conditions of $\$ 2.11$. Suppose in addition that $X / K$ has finite cohomological dimension, and that $K$ is $\sigma$-plain in the sense of $\$ 4.1$. In this situation, as described above,

$$
\begin{array}{cc}
H^{*}(X / K) & \text { is a left } \mathscr{H}(G, K) \text {-module } \\
H^{*}\left(X^{\sigma} / K^{\sigma}\right) & \text { is a left } \mathscr{H}\left(G^{\sigma}, K^{\sigma}\right) \text {-module }
\end{array}
$$

Then we have: 
(a) If we regard these as $\mathscr{H}(G, K)^{\sigma}$-modules (via restriction for (4.4.1) and via $\mathrm{Br}$ for (4.4.2)), then every composition factor of (4.4.2) is also a composition factor of (4.4.1).

(b) Suppose that we are in the setting of $\$ 4.3$ - i.e., suppose that $\mathscr{H}(G, K)$ and $\mathscr{H}\left(G^{\sigma}, K^{\sigma}\right)$ are both commutative integral domains - so the normalized Brauer homomorphism br is defined. Suppose that $\chi: \mathscr{H}\left(G^{\sigma}, K^{\sigma}\right) \rightarrow k$ is a character that appears as an eigenvalue of (4.4.2) (i.e. there exists an element of $H^{*}\left(X^{\sigma} / K^{\sigma}\right)$ annihilated by all $h-\chi(h)$ for $\left.h \in \mathscr{H}\left(G^{\sigma}, K^{\sigma}\right)\right)$.

Then also $\chi \circ$ br appears as an eigenvalue of (4.4.1).

Proof. Note that (a) implies (b). Indeed, suppose that $\chi$ is as in (b). By (a) we have

$$
\chi \circ \mathrm{Br} \text { appears as an eigenvalue of (4.4.1) when restricted to } \mathscr{H}(G, K)^{\sigma} \text {. }
$$

We have seen in $\$ 3.4$ that $(\chi \circ \widetilde{\mathrm{Br}})^{1 / p}$ is the unique extension of $\chi \circ \operatorname{Br}$ from $\mathscr{H}(G, K)^{\sigma}$ to $\mathscr{H}(G, K)$. So, supposing (4.4.3), we see that $(\chi \circ \widetilde{\mathrm{Br}})^{1 / p}$ appears as an eigenvalue of (4.4.1). But this implies that $\chi \circ$ br appears as an eigenvalue of (4.4.1): the isomorphisms

$$
H^{*}(X / K)=H^{*}\left(X / K ; \mathbf{F}_{p}\right) \otimes_{\mathbf{F}_{p}} k, \mathscr{H}(G, K)=\operatorname{Fun}_{G}\left(G / K \times G / K, \mathbf{F}_{p}\right) \otimes_{\mathbf{F}_{p}} k
$$

yield semilinear actions of $\operatorname{Aut}(k)$ on $\mathscr{H}(G, K)$ and $H^{*}(X / K)$. We have also

$$
\alpha^{\tau}\left(h^{\tau}\right)=(\alpha(h))^{\tau}
$$

for $\alpha \in \mathscr{H}, h \in H^{*}(X / K), \tau \in \operatorname{Aut}(k)$. So if $h \in H^{*}(X / K)$ corresponds to the eigenvalue $(\chi \circ \widetilde{\mathrm{Br}})^{1 / p}$ then $h^{\tau}$ corresponds to the eigenvalue $\chi \circ \mathrm{br}$, where $\tau$ is the Frobenius automorphism.

The proof of (a) is an application of "fixed point localization" methods of Smith, Borel, Quillen. We give a treatment here that is well adapted to keeping track of the Hecke action. The statement of the theorem for homology implies the statement for cohomology — let us prove the homology version.

Consider the "Smith double complex"

$$
C_{*}^{\text {Smith }}:=\left[\cdots \longleftarrow{ }_{*} C_{*}(X / K) \stackrel{1-\sigma}{\longleftarrow} C_{*}(X / K) \stackrel{N}{\longleftarrow} C_{*}(X / K)^{1-\sigma} \stackrel{\leftarrow}{\longleftarrow}\right]
$$

The map $\sigma$ is not a map of $\mathscr{H}(G, K)$-modules but it is a map of $\mathscr{H}(G, K)^{\sigma}$-modules, so $C_{*}^{\text {Smith }}$ is a double complex of $\mathscr{H}(G, K)^{\sigma}$-modules. It leads to two spectral sequences of $\mathscr{H}(G, K)^{\sigma}$-modules:

- The spectral sequence ${ }^{h v} E$, in which the differential on the 0th page is the horizontal differential, on the 1st page is the vertical differential.

- The spectral sequence ${ }^{v h} E$, in which the differential on the 0 th page is the vertical differential and on the 1st page is the horizontal differential.

If $C_{*}$ is bounded, then both ${ }^{h v} E$ and ${ }^{v h} E$ converge to the homology of the total complex of $C_{*}^{\text {Smith }}$. Let us abbreviate the horizontal differential (which alternates between $1-\sigma$ or $N$ ) by $d^{h}$ and the vertical differential (which is the standard singular differential on $C_{*}(X / K)$ ) by $d^{v}$. We can compute higher differentials in these spectral sequences by the following standard device. If $x \in{ }^{h v} E_{i j}^{0}$ is an element that survives to ${ }^{h v} E_{i j}^{r}$, and $\left(x_{1}, \ldots, x_{r}\right)$ is a sequence of elements with $x=x_{1}$ and $d^{v}\left(x_{i}\right)=d^{h}\left(x_{i+1}\right)$ for $i<r$, then $d^{v}\left(x_{r}\right)$ is a representative for $d^{r}(x)$.

We complete the proof in three steps:

(1) Degeneration of ${ }^{h v} E$. The ${ }^{h v} E$ spectral sequence is analyzed as follows: 
By equation (4.2.1) the first page ${ }^{h v} E^{1}$ is naturally identified with $C_{*}\left((X / K)^{\sigma}\right)$ i.e.

$$
{ }^{h v} E_{i j}^{1}=C_{j}\left((X / K)^{\sigma}\right) \quad d^{1}: C_{j} \rightarrow C_{j-1} \text { is the singular differential }
$$

It follows that ${ }^{h v} E_{i j}^{2}=H_{j}\left((X / K)^{\sigma}\right)$. Now, if $\zeta \in{ }^{h v} E_{i j}^{0}=C_{j}(X / K)$ has $d^{0}(\zeta)=0$ then, by (4.2.1),

$$
\zeta=\zeta^{\prime}+d^{0} \varepsilon
$$

for some $\zeta^{\prime} \in C_{j}\left((X / K)^{\sigma}\right)$. If $\zeta$ survives to ${ }^{h v} E^{2}$, we must have, in addition, $d^{v} \zeta \in$ $\operatorname{Im}\left(d^{0}\right)$, or equivalently $d^{v} \zeta^{\prime} \in \operatorname{im}\left(d^{0}\right)$. But $d^{v} \zeta^{\prime} \in C_{j-1}\left((X / K)^{\sigma}\right)$; by another application of (4.2.1), $d^{v} \zeta^{\prime}$ is identically zero. In other words, every element of ${ }^{h v} E^{2}$ is represented by a cycle $\zeta^{\prime} \in C_{j}\left((X / K)^{\sigma}\right)$. Then $\left(\zeta^{\prime}, d^{v}\left(\zeta^{\prime}\right)=0,0,0, \ldots, 0\right)$ is a sequence we may use to compute $d^{r}\left(\zeta^{\prime}\right)=0$ for all $r \geq 2$. Thus, ${ }^{h v} E^{2}={ }^{h v} E^{\infty}$.

(2) Compatibility with the Brauer homomorphism. In other words, (1) shows that the homology of the total complex of $C_{*}^{\text {Smith }}$ has a filtration (by $\mathscr{H}(G, K)^{\sigma}$-submodules) whose associated graded is $H_{*}\left((X / K)^{\sigma}\right)$. We claim that our assumptions imply that $X^{\sigma} / K^{\sigma}$ is a union of connected components of $(X / K)^{\sigma}$.

To prove the claim, let $Y \subset X$ be the inverse image of $(X / K)^{\sigma}$. As $K$ acts freely, for each $y \in Y$ there is a unique $\kappa(y) \in K$ such that $y \kappa(y)=\sigma(y)$. The map $\kappa$ is $K$-equivariant for the $\sigma$-twisted conjugation action on $K$. The graph of $\kappa$ is the set of all $(y, k) \in Y \times K$ with $\sigma(y)=y k-$ in particular it is a closed set. The projection of this graph to $Y$ is a homeomorphism. So $\kappa$ is a continuous function $Y \rightarrow K$. It descends to a continuous function

$$
Y / K=(X / K)^{\sigma} \rightarrow \sigma \text {-twisted conjugacy classes for } K,
$$

where we give the right-hand side the quotient topology. But the space of $\sigma$-twisted conjugacy classes for $K$ is a finite set, because we assumed that $K$ is $\sigma$-plain, and because each $\sigma$-twisted conjugacy class is closed in $K$, the topology on this finite set is the discrete topology. It follows that $X^{\sigma} / K^{\sigma}$ is a union of connected components of $(X / K)^{\sigma}$.

Thus, on the first page, ${ }^{h v} E_{i j}^{1}=C_{i}\left((X / K)^{\sigma}\right)$ has $C_{i}\left(X^{\sigma} / K^{\sigma}\right)$ as a vector space summand. By equation (4.2.2) - applied with $\tilde{S}$ equal to the free $K$-set of singular $i$-simplices in $X$ - the action of $\mathscr{H}(G, K)^{\sigma}$ on this summand factors through $\mathrm{Br}$ and it is actually a $\mathscr{H}(G, K)^{\sigma}$-submodule. Passing to homology, we conclude that $H_{*}\left(X^{\sigma} / K^{\sigma}\right)$ is a $\mathscr{H}(G, K)^{\sigma}$-submodule of ${ }^{h v} E_{i j}^{2}$.

(3) Convergence of ${ }^{v h} E^{r}$ In this last step, observe that ${ }^{v h} E_{i j}^{1}=H_{j}(X / K)$ and that since $H_{*}(X / K)$ vanishes in large degrees, we have a convergent spectral sequence

$$
{ }^{v h} E_{i j}^{1}=H_{j}(X / K) \Longrightarrow H_{*}\left(\operatorname{Tot}\left(C^{\text {Smith }}\right)\right)
$$

of $\mathscr{H}(G, K)^{\sigma}$-modules. Therefore by (2), we obtain the desired statement: we have exhibited $H_{*}\left(X^{\sigma} / K^{\sigma}\right)$, as a composition factor of $H_{*}(X / K)$, where both are regarded as modules under $\mathscr{H}(G, K)^{\sigma}$. Indeed, even better: We can identify ${ }^{v h} E_{i j}^{2}$ with the Tate cohomology $\mathrm{T}^{i} H_{j}(X / K)$; and we have thus actually exhibited $H_{*}\left(X^{\sigma} / K^{\sigma}\right)$ as a subquotient of $\mathrm{T}^{*} H_{*}(X / K)$.

\section{CYCLIC GROUP ACTIONS ON LOCALLY SYMMETRIC SPACES}

5.1. Definition. Let $\mathbf{G}, K_{\infty}$ and level structure $K \subset \mathbf{G}\left(\mathbf{A}_{\mathrm{f}}\right)$ be as in $\S 2.3$. Let $[G]_{K}$ denote the double coset space

$$
[G]_{K}:=\mathbf{G}(F) \backslash \mathbf{G}(\mathbf{A}) /\left(K_{\infty} \times K\right)
$$


If $K \cap \mathbf{G}(F)$ is torsion-free, the homology and cohomology of $[G]_{K}$ carry the action of the Hecke algebra $\mathscr{H}\left(\mathbf{G}\left(\mathbf{A}_{\mathrm{f}}\right), K\right)$ described in $\$ 2.11$. For general $K$, one should regard $[G]_{K}$ as an orbifold and take homology and cohomology in this sense, in which case a more careful discussion defines an action of $\mathscr{H}$ as well.

The Hecke algebra $\mathscr{H}\left(\mathbf{G}\left(\mathbf{A}_{\mathrm{f}}\right), K\right)$ is a restricted tensor product over finite places

$$
\mathscr{H}\left(\mathbf{G}\left(\mathbf{A}_{\mathrm{f}}\right), K\right)=\bigotimes_{v}^{\prime} \mathscr{H}\left(G_{v}, K_{v}\right)
$$

where the restricted product is taken with respect to the identity element in $\mathscr{H}\left(G_{v}, K_{v}\right)$. When $V$ is a set of finite places, we write $\mathscr{H}\left(G_{V}, K_{V}\right):=\bigotimes_{v \in V}^{\prime} \mathscr{H}\left(G_{v}, K_{v}\right)$; we sometimes abbreviate this to simply $\mathscr{H}_{V}$.

5.2. Good places. We call a place $v$ good with respect to the algebraic group $\mathbf{G}$, level structure $K$ and prime $p$ if

(i) The residue characteristic of $\mathcal{O}_{v}$ is not equal to $p$

(ii) $\mathbf{G} \times{ }_{F} F_{v}$ is quasi-split over $F_{v}$ and split over an unramified extension of $F_{v}$.

(iii) $K_{v}$ is a hyperspecial subgroup of $G_{v}$. In other words, $K_{v}$ is a maximal compact subgroup of the form $\mathfrak{G}\left(\mathcal{O}_{v}\right)$, where $\mathfrak{G}$ is a reductive smooth model for $\mathfrak{G} \times{ }_{F} F_{v}$ over $\mathcal{O}_{v}$.

For any $K$, all but finitely many places are good. At a good place, $\mathscr{H}\left(G_{v}, K_{v}\right)$ is a commutative integral domain and its characters are understood via the Satake isomorphism; for this, see $\$ 7.2$.

5.3. Characters of the Hecke algebra occurring on cohomology. Suppose given a character $\chi$ of $\mathscr{H}\left(G_{V}, K_{V}\right)$, where $V$ is a set of finite good places. We say " $\chi$ appears in the cohomology of $[G]_{K}$ " if there is $h \in H^{*}\left([G]_{K}\right)$ such that $h$ transforms under $\mathscr{H}\left(G_{V}, K_{V}\right)$ by $\chi$.

The following result shows that it is enough to consider "sufficiently small" level structures, in particular — as long as $V$ excludes at least one finite place - one may always assume that the relevant locally symmetric spaces are manifolds and not merely orbifolds.

5.4. Proposition. Suppose that $K=\prod K_{v}$ and $K^{\prime}=\prod K_{v}^{\prime}$ where $K_{v}^{\prime} \subset K_{v}$ for all $v$ with equality $K_{v}^{\prime}=K_{v}$ for $v \in V$. If $\chi$ appears in the cohomology of $[G]_{K}$ then it also appears in the cohomology of $[G]_{K^{\prime}}$.

Proof. The finite group $K / K^{\prime}$ acts on the cohomology of $[G]_{K^{\prime}}$. For all $v \in V$ we have $K_{v}=K_{v}^{\prime}$, and the actions of $\mathscr{H}_{v}$ and $K / K^{\prime}$ on $H^{*}\left([G]_{K^{\prime}}\right)$ commute. The spectral sequence

$$
E_{2}^{i j}=H^{i}\left(K / K^{\prime} ; H^{j}([G])_{K^{\prime}}\right) \Longrightarrow H^{i+j}\left([G]_{K}\right)
$$

is a spectral sequence of $\mathscr{H}_{V}$-modules. Thus, a character of $\mathscr{H}_{V}$ that occurs in the cohomology of $[G]_{K}$ also occurs in $H^{*}\left(K / K^{\prime} ; H^{*}([G])_{K^{\prime}}\right)$. The bar model for the $K / K^{\prime}$ cohomology of $H^{*}\left([G]_{K^{\prime}}\right)$ shows that the character mus appear in $H^{*}\left([G]_{K^{\prime}}\right)$ itself.

5.5. $\sigma$-action. Now suppose that $\sigma$ acts on $\mathbf{G}$ with order $p$, and set $\mathbf{H}=\mathbf{G}^{\sigma}$. Suppose that $\mathbf{H}$ is connected. We may treat either $\mathbf{G}$ or $\mathbf{H}$ as a special case of the setup of $\$ 5.1$, and we make the following parallel notations and assumptions:

(a) Fix level structures $K$ for $\mathbf{G}$ and $U$ for $\mathbf{H}$, and suppose that $K$ is $\sigma$-stable with fixed points $U=K^{\sigma}$; 
(b) Fix a maximal compact $K_{\infty} \subset \mathbf{G}\left(F_{\infty}\right)$ in such a way that $K_{\infty}$ is $\sigma$-invariant and $K_{\infty}$ intersects $\mathbf{H}\left(F_{\infty}\right)$ in a maximal compact subgroup $U_{\infty}$.

This is always possible: inside the disconnected group $\mathbf{G}\left(F_{\infty}\right) \rtimes\langle\sigma\rangle$, we may find a maximal compact subgroup that contains $U_{\infty} \times\langle\sigma\rangle$, and then we just take its intersection with $\mathbf{G}\left(F_{\infty}\right)$.

(c) Write $[G]_{K}=\mathbf{G}(F) \backslash \mathbf{G}\left(\mathbf{A}_{F}\right) / K_{\infty} K$ and $[H]_{U}=\mathbf{H}(F) \backslash \mathbf{H}\left(\mathbf{A}_{F}\right) / U_{\infty} U$.

(d) $K$ is "sufficiently small", in that $\mathbf{G}(F) \cap K_{\infty} K$ is trivial; so also $\mathbf{H}(F) \cap U_{\infty} U$ is trivial. By Proposition 5.4, this will entail no real loss of generality.

5.6. Proposition. Say that a finite place $v$ is $\sigma$-good with respect to $\mathbf{G}, K, \mathbf{H}, U$ if

(a) $v$ is good with respect to $K$ and $U$ in the sense of $\$ 5.2$, and

(b) $K_{v} \subset G_{v}$ is a $\sigma$-plain subgroup in the sense of $\$ 4.1$.

If $\mathbf{H}$ is connected, then all but finitely many places of $F$ are $\sigma$-good.

We remark that Brian Conrad and Gopal Prasad explained to us how to obtain a much sharper result by reducing to a corresponding assertion for tori.

Proof. Let $\mathfrak{G}$ be a model of $\mathbf{G}$ over $\mathcal{O}_{F}$. We must check that the map $H^{1}\left(\sigma, \mathfrak{G}\left(\mathcal{O}_{v}\right)\right) \rightarrow$ $H^{1}\left(\sigma, G_{v}\right)$ has trivial fiber above the trivial class, for almost all $v$ (it is easy to check the remaining conditions are valid for almost all $v$ ).

Consider the morphism of $\mathcal{O}_{F}$-schemes $g \mapsto g^{-1} \sigma(g)$ from $\mathfrak{G}$ to itself. Its image $I$ is constructible, i.e. a finite union of locally closed sets. On the other hand, it interects the generic fiber of $\mathfrak{G}$ in a closed set $J$ : in characteristic zero, the conjugacy class of $\sigma$ is closed ( [22, Corollary 5.8]: $\sigma$ is automatically semisimple, being of finite order). Let $\bar{J}$ be the closure of $J$ inside $\mathfrak{G}$. The symmetric difference $(\bar{J} \backslash I) \cup(I \backslash \bar{J})$, considered as a subset of $\mathfrak{G}$, is a constructible set which does not intersect the generic fiber. The projection of this symmetric difference to $\operatorname{Spec}\left(\mathcal{O}_{F}\right)$ is (being constructible and disjoint from the generic point) a finite set of closed points.

Let $T$ be the corresponding set of places, together with all places at which $\mathfrak{G}$ or $\mathfrak{G}^{\sigma}$ are not smooth and all places of residue characteristic dividing $p$. In what follows, replace $\mathfrak{G}$ by its restriction to $\mathcal{O}\left[\frac{1}{T}\right]$. Then, by choice of $T$, the image of $g \mapsto g^{-1} \sigma(g)$ is a closed subset $J^{\prime}$ of $\mathfrak{G}$.

We claim that the claim holds for $v \notin T$. Indeed, suppose given $g \in G_{v}$ with the property that $g^{-1} \sigma(g) \in \mathfrak{G}\left(\mathcal{O}_{v}\right)$. We need to verify that the $\mathcal{O}_{v}$-scheme defined by

$$
\mathfrak{X}=\left\{x \in \mathfrak{G}: x^{-1} \sigma(x)=g^{-1} \sigma(g)\right\}
$$

has an $\mathcal{O}_{v}$-point.

Now $\mathfrak{X}$ has a $\overline{\mathbf{F}_{v}}$-point: By assumption, $g^{-1} \sigma(g)$ yields a map $\operatorname{Spec}\left(\mathcal{O}_{v}\right) \rightarrow \mathfrak{G}$ sending the generic point of $\operatorname{Spec}\left(\mathcal{O}_{v}\right)$ to an element of $J^{\prime}$. Because $J^{\prime}$ is closed, the special point of $\operatorname{Spec}\left(\mathcal{O}_{v}\right)$ is also sent to an element of $J^{\prime}$, i.e. there exists $y \in \mathfrak{G}\left(\overline{\mathbf{F}_{v}}\right)$ with $y^{-1} \sigma(y)=g^{-1} \sigma(g)$ modulo $v$, as desired.

Therefore, $\mathfrak{X}$ also has a point over $\mathbf{F}_{v}$, because $\mathfrak{X}\left(\overline{\mathbf{F}_{v}}\right)$ is a torsor under $\mathbf{H}\left(\overline{\mathbf{F}_{v}}\right)$ and Steinberg's theorem [31, Theorem 1.9] says that the Galois cohomology of the connected algebraic group $\mathbf{H}$ is trivial over the finite field $\mathbf{F}_{v}$.

In other words there exists $x \in \mathfrak{G}\left(\mathcal{O}_{v}\right)$ such that

$$
(x g)^{-1} \sigma(g x) \in \Delta_{v}:=\operatorname{ker}\left(\mathfrak{G}\left(\mathcal{O}_{v}\right) \rightarrow \mathfrak{G}\left(\mathbf{F}_{v}\right)\right),
$$

i.e. it defines a class in $H^{1}\left(\sigma, \Delta_{v}\right)$. But $\Delta_{v}$ has pro-order that is relatively prime to $p$, so that class must vanish, i.e. there exists $\delta \in \Delta_{v}$ such that $(x g)^{-1} \sigma(g x)=\delta^{-1} \sigma(\delta)$. In other words, the class of $g^{-1} \sigma(g)=y^{-1} \sigma(y)$ where $y=\delta x^{-1} \in \mathfrak{G}\left(\mathcal{O}_{v}\right)$, as desired. 
5.7. Analysis of connected components. Let $V$ be any nonempty finite set of $\sigma$-good places. Write $K^{(V)}=K_{\infty} \prod_{w \notin V} K_{w}$. We are going to apply the discussion of $\S 4.4$, with

$$
X=\mathbf{G}(F) \backslash \mathbf{G}\left(\mathbf{A}_{F}\right) / K^{(V)}
$$

and the acting groups (“ $G, K$ ” from $\S 4.4$ )

$$
\begin{aligned}
& G_{V}=G \text { from } \S 4.4=\prod_{w \in V} \mathbf{G}\left(F_{w}\right), \\
& K_{V}=K \text { from } \S 4.4=\prod_{w \in V} K_{w},
\end{aligned}
$$

Since we assumed that $\mathbf{G}(F) \cap K_{\infty} K$ is trivial, the group $K_{V}$ acts freely on $X$. The main issue is to precisely analyze how the fixed locus $X^{\sigma}$ is related to $\mathbf{H}$.

Proposition. The natural map $[H]_{U} \rightarrow X^{\sigma} / K_{V}^{\sigma}$ maps $[H]_{U}$ homeomorphically onto a union of components of $X^{\sigma} / K_{V}^{\sigma}$.

Proof. There's a map

$$
\mathfrak{e}: X^{\sigma} / K_{V}^{\sigma} \rightarrow H^{1}(\sigma, \mathbf{G}(F)) \times H^{1}\left(\sigma, K^{(V)}\right)
$$

constructed as follows: For $g \in \mathbf{G}\left(\mathbf{A}_{F}\right)$, the double coset $\mathbf{G}(F) g K^{(V)} \in X$ is $\sigma$-fixed if and only if one can find $\gamma \in \mathbf{G}(F)$ and $\kappa \in K^{(V)}$ such that $\sigma(g)=\gamma g \kappa$ inside $\mathbf{G}\left(\mathbf{A}_{F}\right)$. Consider this equality at a place $w \in V$ : it shows that actually $\gamma=\sigma\left(g_{w}\right) g_{w}^{-1}$, in particular, it satisfies $\sigma^{p-1}(\gamma) \ldots \sigma(\gamma) \gamma=e$. Then computing $\sigma^{p}(g)$ we see that also $\kappa \sigma(\kappa) \ldots \sigma^{p-1}(\kappa)=e$. In other words $\sigma \mapsto \gamma^{-1}$ and $\sigma \mapsto \kappa$ define cocycles in $H^{1}(\sigma, \mathbf{G}(F))$ and $H^{1}\left(\sigma, K^{(V)}\right)$; these classes depend only on the double coset.

The map $\mathfrak{e}$ of (5.7.1) is locally constant. In fact choose $x \in X^{\sigma}$ and a representative $g \in \mathbf{G}\left(\mathbf{A}_{F}\right)$. Let $U_{\infty}$ be a $\sigma$-fixed open neighbourhood of $K_{\infty}$ inside $\mathbf{G}\left(F_{\infty}\right)$ and let $U=U_{\infty} \cdot K$. Suppose $g$ is, as above, so that the double coset $\mathbf{G}(F) g K^{(V)}$ is $\sigma$-fixed, and $\gamma, \kappa$ are as above. Suppose that $g u$ also defines a $\sigma$-fixed element of $X$, i.e.

$$
\sigma(g) \sigma(u)=\gamma^{\prime} g u \kappa^{\prime} \Longrightarrow \gamma g \kappa \sigma(u)=\gamma^{\prime} g u \kappa^{\prime},
$$

and in particular,

$$
\gamma g K \cap \gamma^{\prime} g K \cdot U_{\infty} \cdot U_{\infty}^{-1} \neq \emptyset
$$

Because the action of $\mathbf{G}(F)$ on $\mathbf{G}\left(\mathbf{A}_{F}\right) / K_{\infty} K$ is properly discontinuous and free by assumption (d) of $\S 5.5$, this implies that $\gamma^{\prime}=\gamma$ if $U_{\infty}$ is chosen sufficiently small. (Recall that $K_{\infty}$ is chosen $\sigma$-invariant, and so one may choose $U_{\infty}$ to be an arbitrarily small open neighbourhood of it.)

We also then have $\kappa=u \kappa^{\prime}(\sigma(u))^{-1}$ and thus the corresponding classes in $H^{1}\left(\sigma, K^{(V)}\right)$ are also equal. Indeed, this is now clear for the projection to the latter component of $K^{(V)} \simeq K_{\infty} \times \prod_{w \notin V} K_{w}$; to handle the $K_{\infty}$ component we observe that $H^{1}\left(\sigma, K_{\infty}\right)$ is finite, and for each class in $H^{1}\left(\sigma, K_{\infty}\right)$ the set of representing cocycles is closed; thus the induced topology on $H^{1}\left(\sigma, K_{\infty}\right)$ is the discrete one. Thus, if we take $U_{\infty}$ sufficiently small, the classes $H^{1}\left(\sigma, K_{\infty}\right)$ corresponding to $\kappa_{\infty}$ and $\kappa_{\infty}^{\prime}$ are then forced to be equal.

The natural $[H]_{U} \rightarrow X^{\sigma} / K_{V}^{\sigma}$ is injective: if the double cosets of $h, h^{\prime} \in \mathbf{H}\left(\mathbf{A}_{F}\right)$ map to the same point, we have $h=\gamma h^{\prime} k$ with $\gamma \in \mathbf{G}(F), k \in K^{(V)} K_{V}^{\sigma}$; considering components at a place $w \in V$ we see that $h_{w}=\gamma h_{w}^{\prime} k_{w}$, and in particular $\gamma$ is $\sigma$-invariant; then $k$ too is $\sigma$-invariant and belongs to $\left(K_{\infty} K\right)^{\sigma}=U_{\infty} U$.

Finally, the image of $[H]_{U} \rightarrow X^{\sigma} / K_{V}^{\sigma}$ is, by definition, precisely the fiber of $\mathfrak{e}$ above the trivial class, i.e. a union of connected components. This map from $[H]_{U}$ to its image is now a proper continuous bijection, so a homeomorphism. 
(We do not know a reference for the properness, which uses the reductivity of $\mathbf{H}$; we outline this argument. Let $A^{+}$be the positive cone in the connected real points of a maximal split torus of $\mathbf{H}$. Then reduction theory shows that there is a compact set $\Omega \subset \mathbf{H}\left(\mathbf{A}_{F}\right)$ such that $A^{+} \cdot \Omega$ surjects to $\mathbf{H}(F) \backslash \mathbf{H}\left(\mathbf{A}_{F}\right)$. We are reduced to verifying that $A^{+} \rightarrow \mathbf{G}(F) \backslash \mathbf{G}(\mathbf{A})$ is proper, and in turn that will follow from the properness of $A_{G} \rightarrow \mathbf{G}(F) \backslash \mathbf{G}(\mathbf{A})$, where $A_{G}$ is the group of connected real points of a maximal $F$-split torus inside $\mathbf{G}$. For the last statement, we use the action of the Weyl group of $\mathbf{G}$ to reduce to the case of $A_{G}^{+} \rightarrow \mathbf{G}(F) \backslash \mathbf{G}(\mathbf{A})$, where $A_{G}^{+}$is again a positive cone in $A_{G}$. That statement is again part of reduction theory.)

At this point we are ready to prove the first Theorem from the introduction.

5.8. Theorem. Let $\mathbf{G}, \mathbf{H}, U, K, \sigma$ be as in $\$ 5.5$, and $V$ a nonempty set of $\sigma$-good places. Then $\mathscr{H}\left(G_{V}, K_{V}\right)$ and $\mathscr{H}\left(H_{V}, U_{V}\right)$ are both commutative integral domains, and in particular the normalized Brauer homomorphism br : $\mathscr{H}\left(G_{V}, K_{V}\right) \rightarrow \mathscr{H}\left(H_{V}, U_{V}\right)$ of $\$ 4.3$ is defined. If $\chi: \mathscr{H}\left(H_{V}, U_{V}\right) \rightarrow k$ is an eigenvalue occuring in the cohomology of $[H]_{U}$ then the character $\tilde{\chi}=\chi \circ \mathrm{br}: \mathscr{H}\left(G_{V}, K_{V}\right) \rightarrow k$ occurs in the cohomology of $[G]_{K}$.

Proof. The fact that $\mathscr{H}\left(G_{V}, K_{V}\right)$ and $\mathscr{H}\left(H_{V}, U_{V}\right)$ are commutative integral domains is well-known (at least in the context where the coefficient ring is $\mathbf{C}$ rather than $k$, but the same proof works); we summarize the proof in Theorem 7.2.

It suffices to prove the theorem when $V$ is finite. For suppose that $V$ is infinite and the theorem is false, i.e. $\tilde{\chi}$ doesn't occur in the cohomology of $[G]_{K}$. Since that cohomology is finite-dimensional, there is certainly a finite subset $V^{\prime} \subset V$ such that the restriction of $\tilde{\chi}$ to $\mathscr{H}\left(G_{V^{\prime}}, K_{V^{\prime}}\right)$ doesn't occur in the cohomology of $[G]_{K}$.

Now suppose $V$ is finite. As we saw above, $H^{*}\left([H]_{U}\right)$ is a direct summand of $H^{*}\left(X^{\sigma} / K_{V}^{\sigma}\right)$, where $X$ is as above. Indeed, it is even a $\mathscr{H}\left(H_{V}, U_{V}\right)$-submodule, as is clear by inspection. Now apply Theorem 4.4 (and use Theorem 7.2, applied to both $\mathbf{G}$ and $\mathbf{H}$, to check the conditions.)

\section{REPRESENTATION THEORY}

Let $\mathbf{G}, K, \mathbf{H}, U$ be as in $\S 5.5$. For any finite place $v$ the Hecke algebras $\mathscr{H}\left(G_{v}, K_{v}\right)$ and $\mathscr{H}\left(H_{v}, U_{v}\right)$ describe portions of the category of representations of $G_{v}$-modules and $H_{v}$-modules. In this section we make precise a sense in which the Brauer homomorphism of $\$ 4.2$ "lifts" to a functor between categories of representations. This is relevant both to understand the situation at ramified places and for the proof of the Theorem of $\S 8.1$.

6.1. Linkage and the Brauer homomorphism. Let $\mathbf{G}, K, \mathbf{H}, U$ be as in $\S 5.5$. Fix a finite place $v$ of $F$, of residue characteristic $\neq p$. We do not require that $K_{v}$ is maximal compact. In particular, $\mathscr{H}\left(G_{v}, K_{v}\right)$ is not necessarily commutative.

We consider irreducible $k$-linear representations $\Pi$ of $G_{v}$. These are always understood to be continuous, i.e. every vector in $\Pi$ has open stabilizer (often called "smooth.") We will only consider admissible representations: $\Pi^{K^{\prime}}$ is finite-dimensional over $k$ for every compact open subgroup $K^{\prime} \subset G$. Say that such a representation is $\sigma$-fixed if it is isomorphic to $\Pi \circ \sigma$.

Proposition. If $\Pi$ is $\sigma$-fixed, then there is a unique action of $\sigma$ on $\Pi$ compatible with the $\sigma$-action on $G_{v}$.

Proof. If $A$ is a $k$-linear isomorphism from $\Pi$ to itself that intertwines $\Pi$ with $\Pi \circ \sigma$, then we claim $A^{p}$ must be a scalar. If that scalar is $\lambda$, then $\sigma=\lambda^{-1 / p} A$ is a $\sigma$-action on $\Pi$ compatible with the $\sigma$-action on $G_{v}$. 
To prove the claim, choose a prime-to- $p$ open subgroup $K_{v}^{0} \subset K_{v}$. Then $\Pi^{K_{v}^{0}}$ is a finitedimensional irreducible representation of the Hecke algebra $\mathscr{H}\left(G_{v}, K_{v}\right)$, so by Schur's lemma $A^{p}$ acts as a scalar on $\Pi^{K_{v}^{0}}$. Since the image of the action map $G_{v} / K_{v}^{0} \times \Pi^{K_{v}^{0}} \rightarrow \Pi$ generates $\Pi, A^{p}$ must act by the same scalar on the entirety of $\Pi$.

For a $\sigma$-fixed $\Pi$ with its action of $\sigma$, we may then consider the Tate cohomology $\mathrm{T}^{i} \Pi$ for $i \in\{0,1\}$. It carries an action of $H_{v}$.

6.2. Definition. We say that an irreducible representation $\pi$ of $H_{v}$ is linked with $\Pi$ if the Frobenius-twist $\pi^{(p)}$ (see $\S 2.1$ ) occurs as a Jordan-Holder constituent of $\mathrm{T}^{0}(\Pi)$ or $\mathrm{T}^{1}(\Pi)$.

As a motivating example, which may explain the role of the Frobenius-twist: take $\mathbf{G}=$ $\mathbf{H}^{p}$ and $\sigma$ to act by cyclic permutation. Then the irreducible representation $\pi_{v}$ of $H_{v}$ is linked with the irreducible representation $\pi_{v}^{\otimes p}$ of $G_{v} \simeq H_{v}^{p}$.

The notion of linkage is a representation theoretic version of the Brauer homomorphism: Let $\Pi$ be a $\sigma$-fixed representation of $G_{v}$. We may apply $\mathrm{T}^{*}$ to the $\mathscr{H}\left(G_{v}, K_{v}\right)$-module $\Pi^{K_{v}}$. The $\sigma$-equivariant inclusion map $\Pi^{K_{v}} \rightarrow \Pi$ induces $\mathrm{T}^{*}\left(\Pi^{K_{v}}\right) \rightarrow \mathrm{T}^{*}(\Pi)$, which in fact takes values in the $\mathscr{H}\left(H_{v}, U_{v}\right)$-module $\mathrm{T}^{*}(\Pi)^{U_{v}}$.

We now suppose that $H_{v} / U_{v}=\left(G_{v} / K_{v}\right)^{\sigma}$, as in $\S 4$. 1, i.e. $K_{v}$ is $\sigma$-plain in the notation of that section. Then the (unnormalized) Brauer homomorphism $\operatorname{Br}: \mathscr{H}\left(G_{v}, K_{v}\right)^{\sigma} \rightarrow$ $\mathscr{H}\left(H_{v}, U_{v}\right)$ is compatible with linkage in that the diagram

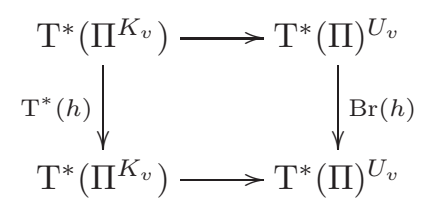

commutes for any $h \in \mathscr{H}\left(G_{v}, K_{v}\right)^{\sigma}$.

Proof. We give the proof for $\mathrm{T}^{0}$. If $x \in \Pi^{K_{v}}$ is $\sigma$-fixed, then the image of $x+N\left(\Pi^{K_{v}}\right)$ in $\mathrm{T}^{0}(\Pi)^{U_{v}}$ is $x+N(\Pi)$, and to verify (6.2.1) we have to show that the equation

$$
\operatorname{Br}(h) *(x+N(\Pi))=(h * x)+N(\Pi)
$$

holds. The left-hand side is

$$
\begin{aligned}
& \sum_{g U_{v} \in H_{v} / U_{v}} \operatorname{Br}(h)\left(U_{v}, g U_{v}\right) g(x+N(\Pi)) \\
= & \sum_{g U_{v} \in H_{v} / U_{v}} h\left(K_{v}, g K_{v}\right)(g x+N(\Pi))
\end{aligned}
$$

and the right-hand side is

$$
\left(\sum_{g K_{v} \in G_{v} / K_{v}} h\left(K_{v}, g K_{v}\right) g x\right)+N(\Pi)
$$

so (6.2.1) reduces to checking

$$
\sum_{g K_{v} \in G_{v} / K_{v}-H_{v} / U_{v}} h\left(K_{v}, g K_{v}\right) g x \in N(\Pi)
$$

Since we have assumed $\left(G_{v} / K_{v}\right)^{\sigma}=H_{v} / U_{v}, \sigma$ acts freely on the set indexing the sum, which therefore does belong to $N(\Pi)$. A similar computation shows (6.2.1) holds for $x+(1-\sigma)\left(\Pi^{K_{v}}\right) \in \mathrm{T}^{1}\left(\Pi^{K_{v}}\right)$. 
6.3. Conjectures. It seems very reasonable to believe that

Let $\Pi$ be a $\sigma$-fixed irreducible admissible representation of $G_{v}$. Then $\mathrm{T}^{*} \Pi$

is of finite length as an $\mathrm{H}_{v}$-representation.

The conjecture is motivated by the analogy with Eisenstein series formulated in the introduction. If the functor $\mathrm{T}^{i} \Pi$ should be seen as an analog of the Jacquet functor, then the Conjecture is a counterpart to the fact that the Jacquet functor carries admissibles to admissibles [13, Theorem 3.3.1]. The analogy, together with computations we have carried out in the case of depth zero base change for $\mathrm{GL}_{n}$, suggests another conjecture, which we will leave in a slightly less precise form:

Linkage is compatible with the Langlands functorial transfer associated to a $\sigma$-dual homomorphism ${ }^{L} \hat{\psi}:{ }^{L} \hat{H} \rightarrow{ }^{L} \hat{G}(\S 1.2, \S 7.7)$

In particular, if the $\sigma$-fixed representation $\Pi$ of $G_{v}$ is linked with the representation $\pi$ of $H_{v}$, we should expect ${ }^{L} \hat{\psi}$ to carry the Langlands parameter of $\pi$ to the Langlands parameter of $\Pi$. In other words: Just as the Jacquet functor realizes functoriality between an $L$ group and a Levi subgroup, we expect that the Tate cohomology functor should realize functoriality for the $\sigma$-dual homomorphism of $\$ 7.7$.

6.4. Ramified places. Fix a finite set $V$ of places of $F$ and a level structure $K \subset \mathbf{G}$, where each place $v \in V$ is good (\$5.2) with respect to $K$.

Let $S$ be a finite set of finite places, disjoint from $V$, and put $G_{S}=\prod_{w \in S} G_{w}$. Consider all level structures $K^{\prime}$ that agree with $K$ away from the set $S$, that is to say,

$$
K^{\prime}=\prod_{v \in S} K_{v}^{\prime} \cdot \prod_{v \notin S} K_{v} .
$$

The $V$-Hecke algebra $\mathscr{H}\left(G_{V}, K_{V}\right)$ is a commutative integral domain acts on the cohomology of $[G]_{K^{\prime}}$. Let $\chi: \mathscr{H}\left(G_{V}, K_{V}\right) \rightarrow k$ be a homomorphism. We may form the $G_{S}$-module

$$
\pi(\chi):=\chi \text {-component of } \underset{K^{\prime}}{\lim } H^{*}\left([G]_{K^{\prime}}\right)
$$

where by $\chi$-component we mean in fact the localization at the maximal ideal defined by $\chi$, i.e. the generalized eigenspace corresponding to $\chi$. Strictly speaking, as we have defined it, this depends on both $\chi$ and $V$, but we have suppressed the dependence on $V$ in the notation.

The precise determination of $\pi(\chi)$ is an interesting and difficult question; it is the subject of the $\bmod p$ Langlands correspondence [18]. In any case, $\pi(\chi)$ and all of its irreducible subquotients are admissible: if we take $K^{\prime}$ small enough that $K_{S}^{\prime}:=\prod_{v \in S} K_{v}^{\prime}$ has pro-order that is prime-to- $p$, then $\pi(\chi)^{K_{S}^{\prime}}$ is identified with the $\chi$-component of cohomology of $H^{*}\left([\mathbf{G}]_{K^{\prime}}\right)$. If we shrink $K^{\prime}$ further, we may ensure that $[\mathbf{G}]_{K^{\prime}}$ is a manifold and has finite-dimensional cohomology.

We are ready to formulate the exact relationship between linkage and the functoriality associated to a $\sigma$-dual homomorphism:

6.5. Theorem. Let $\mathbf{G}, \mathbf{H}, K, U, \sigma$ be as in $\$ 5.5$, and suppose $\mathbf{G}$ is semisimple and $\mathbf{H}$ is connected. Let $V$ be a finite set of $\sigma$-good places (see Proposition 5.6) and $S$ a finite set of finite places disjoint from $V$ and all primes above $p$.

Let $\chi: \mathscr{H}\left(H_{V}, U_{V}\right) \rightarrow k$ be a character, $\psi=\chi \circ$ br. Let $\pi=\pi_{\chi}$ and $\Pi=\Pi(\psi)$ be the representations of (respectively) $H_{S}$ and $G_{S}$ attached to $\chi$ as in \$6.4. Then any irreducible subquotient of the $H_{S}$-module $\pi(\chi)$ is linked with an irreducible subquotient of the $G_{S}$-module $\Pi(\psi)$. 
Note a minor weakness compared to Theorem 5.8: the set $V$ above is required to be finite. It is likely this can be relaxed, and it seems harmless in practice. More seriously one could ask for a more precise statement - for example, a complete determination of one space in terms of the other, but we do not pursue this here.

Proof. Let $K^{\prime} \leqslant G_{S}$ be an open compact subgroup as above (see around (6.4.1)), now assumed $\sigma$-stable; let $U^{\prime}=\left(K^{\prime}\right)^{\sigma} \leqslant H_{S}$.

We proceed just as in $\$ 5.7$ and Theorem 5.8 but in cohomology rather than homology. That furnishes an embedding of

$$
H^{*}\left([H]_{U^{\prime}}\right) \text { as a subquotient of } \mathrm{T}^{*} H^{*}\left([G]_{K^{\prime}}\right)
$$

equivariantly for the action of $\mathscr{H}\left(H_{V}, U_{V}\right)$. In fact, we can do this compatibly at all levels at once, thus embedding $\lim _{\longrightarrow} H^{*}\left([H]_{U^{\prime}}\right)$ as a subquotient of $\mathrm{T}^{*}\left(\underline{\lim } H^{*}\left([G]_{K^{\prime}}\right)\right)$ in an $H_{S} \times \mathscr{H}\left(H_{V}, U_{V}\right)$-equivariant fashion. We explicate this a little:

Proceed as in the proof of Theorem 5.8, but form the associated "Smith double complex" from the direct limit of cochain complexes for the $[G]_{K^{\prime}}$. The sequences are convergent because the cohomological dimension of $[G]_{K^{\prime}}$ is bounded independent of $\left[K^{\prime}\right]$. Our reasoning as before shows that the $h v$-complex converges to $\lim _{\longrightarrow} H^{*}\left([G]_{K^{\prime}}^{\sigma}\right)$ whereas the $E^{2}$ term of the $v h$-complex is $\lim _{\mathrm{T}} \mathrm{T}^{*} H^{*}\left([G]_{K^{\prime}}\right)$. Moreover, $\lim _{\longrightarrow} H^{*}\left([H]_{U^{\prime}}\right)$ is an $H_{S} \times \mathscr{H}\left(H_{V}, K_{V}\right)$-summand of $\lim ^{*}\left([G]_{K^{\prime}}^{\sigma}\right)$.

Now localizing at a character of $\overrightarrow{\mathscr{H}}\left(H_{V}, K_{V}\right)$ we see that (as $H_{S}$-representations)

Any irreducible constituent $\tau$ of $\pi(\chi)$ is a composition factor of $\mathrm{T}^{*} \Pi\left(\psi^{\prime}\right)$

where $\psi^{\prime}=\chi \circ \mathrm{Br}$ is considered as a character of $\mathscr{H}\left(G_{V}, K_{V}\right)^{\sigma}$. By an argument with Frobenius acting on the coefficients, similar to that given earlier, we see that $\tau^{(p)}$ is a composition factor of $\mathrm{T}^{*} \Pi\left(\left(\psi^{\prime}\right)^{p}\right)$. Finally, because $\chi \circ \mathrm{br}$ is the unique extension of $\left(\psi^{\prime}\right)^{p}$ to $\mathscr{H}\left(G_{V}, K_{V}\right)$, we see that $\Pi\left(\left(\psi^{\prime}\right)^{p}\right)=\Pi(\psi)$.

\section{SATAKE PARAMETERS}

In this background section, we recall the Satake isomorphism and the notion of modularity. Then we reformulate the Brauer map in terms of Satake parameters. This section is primarily to set up notation and give references for results which are standard over $\mathbf{C}$ but less so over $k$.

However the formulation of Theorem 7.9 and the accompanying discussion of the $c$ group may be of independent interest. Although Theorem 7.9 points the way to the most intrinsic way of formulating our results, we do not use it in the rest of the paper - sticking instead to the $L$-group and taking the $a d$ hoc approach to the various square roots that occur. This is enough for our purposes, and can be readily matched with the existing literature.

7.1. Restricted Weyl group. Let $v$ be a good place for $\mathbf{G}$, and let $A_{v} \subset B_{v}$ be a maximally split torus and Borel subgroup of $G_{v}$; let $\mathbf{A}_{v} \subset \mathbf{B}$ be the corresponding algebraic groups, and $\mathbf{T}$ the quotient torus of $\mathbf{B}$. The "restricted Weyl group" of $\mathbf{G}$ at $v$ is the quotient $N_{G_{v}}\left(A_{v}\right) / Z_{G_{v}}\left(A_{v}\right)$, i.e. the normalizer of $A_{v}$ divided by the centralizer of $A_{v}$. We denote it by $W_{0, v}$. The correspondence between unramified characters of $T_{v}$ and splittings of $\hat{T}(k) \rtimes \operatorname{Frob}_{v} \rightarrow\left\langle\operatorname{Frob}_{v}\right\rangle$ from $\S 2.9$ is compatible with the natural action of $W_{0, v}$ on each side.

The restricted Weyl group also acts on the dual torus $\hat{A}_{v}$ to $A_{v}$. We will need the following assertion: 
Proposition. The action of any $w \in W_{0, v}$ on $\hat{A}_{v}$ is induced by an element $n \in \hat{G}(k)$ normalizing $\hat{T}$ and fixed by Frob $_{v}$.

This result is proven in [4, Lemma 6.2] over the complex numbers.

Proof. As in $[4, \S 6.1]$ we must show that each Frob $_{v}$-fixed class in the Weyl group of

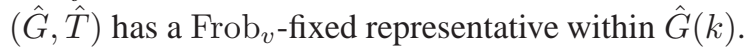

It is proven by Steinberg [31, p 173] that $\sigma$-fixed points of the Weyl group $\hat{W}$ are generated by basic reflections $W_{D}$ indexed by orbits $D$ of Frob on simple roots on $(\hat{G}, \hat{T})$. The basic reflection $W_{D}$ is characterized (item (3) of loc. cit.) as the unique element of $\left\langle w_{\alpha}\right\rangle_{\alpha \in D} \subset \hat{W}$ with the property that $W_{D}$ sends $D$ setwise into $-D$. Equivalently, $W_{D}$ is the long element of the Weyl of the Levi subgroup $\hat{M}_{D}$ obtained when we adjoin each of the $\alpha \in D$ to $\hat{T}$.

We are reduced to a verification inside such a Levi group $\hat{M}$. This is a reductive group $\hat{M}$, equipped with a pinning, and the pinned automorphism Frob ${ }_{v}$ acts transitively on simple roots. We need to produce a representative for the long Weyl group element that is Frob ${ }_{v}$-fixed. It suffices to produce such a representative inside the derived group $[\hat{M}, \hat{M}](k)$, and then inside $\hat{M}^{\prime}(k)$ where $\hat{M}^{\prime}$ is the simply connected cover of that derived group. The question can then be analyzed on each simple factor of $\left(\hat{M}^{\prime}, \operatorname{Frob}_{v}\right)$. The only nontrivial cases are as follows:

(a) $\hat{M}^{\prime}=\mathrm{SL}(3)$ : the pinned automorphism of $\mathrm{SL}(3)$ is given by $g \mapsto \omega\left(g^{t}\right)^{-1} \omega$, where $\omega=\left(\begin{array}{ccc}0 & 0 & 1 \\ 0 & -1 & 0 \\ 1 & 0 & 0\end{array}\right)$. This fixes a representative for the long Weyl group element, namely $\omega$ itself.

(b) $\hat{M}^{\prime}=\hat{M}_{0}^{r}$ and Frob ${ }_{v}$ permutes the simple factors. Note that Frob $_{v}^{r}$ still acts transitively on the simple roots of $\hat{M}_{0}$, and by what we showed above, there is a Frob $v^{r}$ fixed representative $w \in \hat{M}_{0}$ for the long Weyl element; then $(w, \operatorname{Frob}(w), \ldots) \in$ $\hat{M}_{0}^{r}$ gives the desired representative.

7.2. The Satake isomorphism. We now describe the Satake isomorphism. We begin with a statement of the main ingredients, but presented "over $k$ " and with no choices of square roots made. For this statement, we will require the following twisted action of the Weyl group $W_{0, v}$ on $\hat{A}_{v}$ :

$$
w * a=w a \cdot \sqrt{\frac{\Sigma_{G}^{*}}{w \Sigma_{G}^{*}}}\left(q_{v}\right) \quad \text { for } w \in W_{0} \text { and } a \in \hat{A}_{v}(k)
$$

and $\Sigma_{G}^{*}$ is the co-character of $\hat{T}$ given by the sum of all positive coroots. Note that $\Sigma^{*} / w \Sigma_{G}^{*}$ is divisible by 2 in that cocharacter lattice; thus $\sqrt{\frac{\Sigma_{G}^{*}}{w \Sigma_{G}^{*}}}\left(q_{v}\right)$ makes sense, and we can then project to $\hat{A}_{v}$ via $\hat{T} \rightarrow \hat{A}_{v}$.

Theorem. Let $G_{v}=\mathbf{G}\left(F_{v}\right)$ be a reductive v-adic group and let $K_{v} \subset G_{v}$ be a maximal compact subgroup satisfying the conditions of $\$ 5.2$, which is "in good position" with respect to $A_{v}$, i.e. $A_{v} \cap K_{v}$ is a maximal compact subgroup of $A_{v}$. Let $\hat{A}_{v}$ be as in $\$ 2.9$, \$7.1. The following hold: 
(i) There is a natural isomorphism

$$
\mathscr{H}\left(G_{v}, K_{v}\right) \stackrel{\sim}{\longrightarrow} \quad \begin{gathered}
\left(W_{0, v}, *\right) \text {-invariant regular } \\
\text { functions on } \hat{A}_{v}
\end{gathered}
$$

In particular, $\mathscr{H}\left(G_{v}, K_{v}\right)$ is a commutative integral domain.

(ii) There is a natural identification

$$
\begin{array}{ccc}
\left(W_{0, v}, \cdot\right) \text {-invariant regular } \\
\text { functions on } \hat{A}_{v} & \sim & \begin{array}{c}
\text { regular functions on } \\
\hat{G} \rtimes \operatorname{Frob}_{v} / / \hat{G} .
\end{array}
\end{array}
$$

where the $W_{0, v}$-action on $\hat{A}_{v}$ is now the usual one.

Proof of (i). We will prove (i) here, and (ii) in \$7.3. There does not seem to be a reference with coefficients in $k$, so we give some details. We emphasize that we are working with characteristic $p$ coefficients and no square roots are chosen.

We will deduce (i) from some properties of the standard Satake isomorphism (i.e. the Satake isomorphism over $\mathbf{C}$ ). It is a standard observation that the coefficients of this isomorphism can be shrunk from $\mathbf{C}$ to $\mathbf{Z}\left[q_{v}^{ \pm 1 / 2}\right] \subset \mathbf{R}$ (see e.g. [20]). By using the modified $W_{0, v}$-action of (7.2.1), we can further shrink the coefficients to $\mathbf{Z}\left[q_{v}^{ \pm 1}\right] \subset \mathbf{Q}$.

Let us first recall the standard Satake isomorphism. Let $B_{v}$ be the $F_{v}$-points of a Borel containing $A_{v}$, and $N_{v}$ the points of its unipotent radical. Let $\delta_{\mathbf{R}_{>0}}$ be the modular character of the Borel, i.e. the composite

$$
B_{v} \rightarrow F_{v}^{*} \rightarrow q_{v}^{\mathbf{Z}} \subset \mathbf{R}_{>0},
$$

where the first map is the sum $\Sigma_{G}$ of all positive roots, evaluated on $F_{v}$-points. Let $\delta_{\mathbf{R}_{>0}}^{1 / 2}$ denote the positive square root of $\delta_{\mathbf{R}_{>0}}$. The usual Satake transform $f \mapsto \mathcal{S}(f)$ produces from $f \in \mathscr{H}\left(G_{v}, K_{v}\right)$ a function $\mathcal{S}(f)$ on $A_{v}$ given by

$$
\mathcal{S}(f)(t)=\delta_{\mathbf{R}_{>0}}^{1 / 2}(t) \cdot \int_{N_{v}} f\left(K_{v}, \operatorname{tn} K_{v}\right) d n
$$

where the measure $d n$ is normalized so that $N_{v} \cap K_{v}$ has mass 1 . Then $\mathcal{S}(f)$ is compactly supported, and constant on $\left(A_{v} \cap K_{v}\right)$-cosets. We may therefore regard $\mathcal{S}$ as an element of the group ring $\mathbf{C}\left[X_{*}\left(\mathbf{A}_{v}\right)\right]=\mathbf{C}\left[X^{*}\left(\hat{A}_{v}\right)\right]$. As such [20] $\mathcal{S}$ defines a ring isomorphism from $\mathscr{H}$ to the $W_{0, v}$-invariant subring of $\mathbf{C}\left[X^{*}\left(\hat{A}_{v}\right)\right]$, where $W_{0, v}$ acts as in $\S 7.1$; moreover, [21, Lemma 10.2.1], with respect to the basis of double $K_{v}$-cosets in the domain, and of $W_{0, v}$-orbits on $X^{*}\left(\hat{A}_{v}\right)$ in the codomain, $\mathcal{S}$ is upper triangular and the diagonal entries belong to $\mathbf{Z}\left[q_{v}^{ \pm 1 / 2}\right]$. In other words, $\mathcal{S}$ defines an algebra isomorphism

$$
\mathscr{H}\left(G_{v}, K_{v}\right) \text { with } \mathbf{Z}\left[q_{v}^{ \pm 1 / 2}\right] \text {-coefficients } \stackrel{\sim}{\rightarrow} \mathbf{Z}\left[q_{v}^{ \pm 1 / 2}\right]\left[X^{*}\left(A_{v}\right)\right]^{W_{0, v}}
$$

where the $W_{0, v}$-action on $X^{*}\left(\hat{A}_{v}\right)$ is the "untwisted" one from $\S 7.1$.

One obtains a form of the Satake isomorphism over $k$ by tensoring with $k$, but this requires choosing an embedding $\mathbf{Z}\left[q_{v}^{ \pm 1 / 2}\right] \rightarrow k$, i.e. choosing a square root of $q_{v}$ in $k$. To avoid that, define a modified Satake transform $\mathcal{S}^{*}$ by

$$
\mathcal{S}^{*}(f)(t)=\int f\left(K_{v}, \operatorname{tn} K_{v}\right) d n
$$


i.e. $\mathcal{S}^{*}:=\delta^{-1 / 2} \mathcal{S}$. Then $\mathcal{S}^{*}$ is also an injective ring homomorphism with values in $\mathbf{C}\left[X^{*}\left(\hat{A}_{v}\right)\right]$. Since the $*$-action of $W_{0, v}$ on $\mathbf{C}\left[X^{*}\left(\hat{A}_{v}\right)\right](7.2 .1)$ sends $\chi \in X^{*}\left(\hat{A}_{v}\right)$ to

$$
\begin{aligned}
w * \chi & =w \chi \cdot q_{v}^{\left\langle\Sigma_{G}^{*}-w^{-1} \Sigma_{G}^{*}, \chi\right\rangle / 2} \\
& =w \chi \cdot q_{v}^{\left\langle w \Sigma_{G}^{*}-\Sigma_{G}^{*}, w \chi\right\rangle / 2},
\end{aligned}
$$

and we deduce that $\mathcal{S}^{*}$ is an isomorphism onto the $\left(W_{0, v}, *\right)$-invariant subring of $\mathbf{C}\left[X^{*}\left(\hat{A}_{v}\right)\right]$.

For the rest of the proof of (i), let us write $\mathbf{Z}^{\prime}:=\mathbf{Z}\left[q_{v}^{-1}\right]$ and $\mathbf{Z}^{\prime \prime}:=\mathbf{Z}\left[q_{v}^{-1 / 2}\right]$, for short. The $*$-action of $W_{0, v}$ on $\mathbf{C}\left[X^{*}\left(\hat{A}_{v}\right)\right]$ clearly leaves the subrings $\mathbf{Z}^{\prime}\left[X^{*}\left(\hat{A}_{v}\right)\right]$ and $\mathbf{Z}^{\prime \prime}\left[X^{*}\left(\hat{A}_{v}\right]\right.$ stable, and (d) for $\mathcal{S}$ implies that $\mathcal{S}^{*}$ lies in $\mathbf{Z}^{\prime \prime}\left[X^{*}\left(\hat{A}_{v}\right)\right]$. To conclude that $\mathcal{S}^{*}$ is an isomorphism over $\mathbf{Z}^{\prime}$ it is only necessary to check that $\mathcal{S}^{*}$ carries the basis element indexed by $K_{v} t_{0} K_{v}$ into $\mathbf{Z}^{\prime}\left[X^{*}\left(\hat{A}_{v}\right)\right]$, i.e. to check that the measure of $n \in N_{v}$ with $K_{v} \operatorname{tn} K_{v}=K_{v} t_{0} K_{v}$ lies in $\mathbf{Z}^{\prime}$; that follows from the normalization of $d n$.

7.3. Invariant theory lemma. The proof of part (ii) of the Theorem of $\S 7.2$ depends on the following lemma:

Lemma. Every $W_{0}$-invariant function on $\hat{A}$ arises from a $\hat{G}$-invariant function on $\hat{G} \rtimes$ Frob $_{v}$

Proof. We will actually show that the ring of $W_{0}$-invariant regular functions on $\hat{A}$ is spanned by traces of representations of $\hat{G} \rtimes \operatorname{Frob}_{v}$. (It will follow that functions on $\hat{G} \rtimes\left\langle\operatorname{Frob}_{v}\right\rangle / / \hat{G}$ are spanned by traces of representations of $\hat{G} \rtimes\left\langle\operatorname{Frob}_{v}\right\rangle$.)

The ring of regular functions on $\hat{A}$ is naturally identified with the group ring $k\left[X^{*}(\hat{T})_{v}^{\text {Frob }}\right]$. The $W_{0, v}$-invariant regular functions have a basis parameterized by $W_{0, v}$-orbits on $X^{*}(\hat{T})^{\operatorname{Frob}_{v}}$.

Every $W_{0, v}$-orbit on $X^{*}(\hat{T})^{\text {Frob }_{v}}=X_{*}(\mathbf{T})^{\text {Frob }_{v}}$ contains a dominant element: if we look on the dual side, the relative Weyl group for A (i.e., the Weyl group of the relative root system, that might not be reduced) has a relatively dominant element in its orbit, which implies it is dominant considered as a cocharacter of $\mathbf{T}$ - although it may lie on a wall. Let us denote the basis element corresponding to $W_{0, v} \nu$, where $\nu$ is dominant, by $\omega_{\nu}$.

For each dominant weight $\nu$, let $V_{\nu}$ denote the corresponding Weyl module for $\hat{G}$, i.e. by Borel-Weil

$$
V_{\nu}=H^{0}(\hat{G} / \hat{B} ; \mathcal{O}(\nu))
$$

Note that $V_{\nu}$ need not be irreducible, since we are in characteristic $p$, but it does not matter for us. If $\nu$ is Frob -invariant then (as Frob $_{v}$ leaves $\hat{B}$ stable) the line bundle $\mathcal{O}(\nu)$ acquires a $\hat{G} \rtimes\left\langle\right.$ Frob $\left._{v}\right\rangle$-equivariant structure, and $V_{\nu}$ is canonically a $\hat{G} \rtimes\left\langle\right.$ Frob $\left._{v}\right\rangle$-module.

Let $W$ be the full Weyl group for $\hat{T}$, i.e. the quotient $N(\hat{T}) / \hat{T}$. Let $|W \nu| \subset X^{*}(\hat{T})$ denote the convex hull of the $W$-orbit of $\nu$. For $\lambda \in|W \nu|$, let $V_{\nu}(\lambda)$ denote the corresponding weight space of $V(\nu)$. For $t \in \hat{T}$, we compute

$$
\chi_{\nu}\left(t \rtimes \operatorname{Frob}_{v}\right)=\sum_{\lambda \in|W \nu|^{\text {Frob }_{v}}} \lambda(t) \operatorname{Tr}\left(\left.\operatorname{Frob}_{v}\right|_{V_{\nu}(\lambda)}\right)
$$

(where $|W \nu|^{\text {Frob }_{v}}$ is the Frob $_{v}$-fixed elements of $|W \nu|$ ). We will show that

$$
\sum_{\lambda \in(W \nu)^{\mathrm{Frob}_{v}}} \lambda(t) \operatorname{Tr}\left(\left.\operatorname{Frob}_{v}\right|_{V_{\nu}(\lambda)}\right)=\operatorname{Tr}\left(\left.\operatorname{Frob}_{v}\right|_{V_{\nu}(\nu)}\right) \omega_{\nu}
$$

The left-hand side is the dominant term of the right-hand side of (7.3.1), so that (7.3.2) implies

$$
\chi_{v}\left(t \rtimes \operatorname{Frob}_{v}\right)=\operatorname{Tr}\left(\left.\operatorname{Frob}_{v}\right|_{V_{\nu}(\nu)}\right) \cdot \omega_{\nu}+\sum_{\nu^{\prime}} a_{\nu^{\prime}} \omega_{\nu^{\prime}}
$$


where every $\nu^{\prime} \in X^{*}(\hat{T})^{\operatorname{Frob}_{v}}$ has the property that $\left|\nu^{\prime}\right|<|\nu|$. After observing that $\operatorname{Tr}\left(\left.\operatorname{Frob}_{v}\right|_{V_{\nu}(\nu)}\right)$ is nonzero and $V_{\nu}(\nu)$ is one-dimensional, it follows by induction that $\omega_{\nu}$ can be written as a linear combination of characters of $V_{\nu^{\prime}}$, where $\nu^{\prime} \in X^{*}(T)$ and $\nu^{\prime} \leq \nu$.

Let us prove (7.3.2). We may write $W \nu$ as $W / W_{\nu}$, where $W_{\nu}$ is the $\mathrm{Frob}_{v}$-stable parabolic subgroup fixing $\nu$. Recall that each coset of $W_{\nu}$ has a minimum element in the

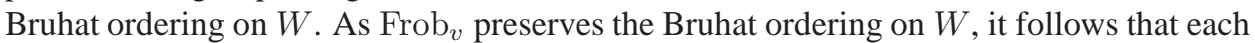
Frob $_{v}$-fixed coset is represented by a Frob ${ }_{v}$-fixed element of $W$, i.e. by an element of $W_{0}$. As Frob ${ }_{v}$ and $W_{0}$ commute, the trace $\operatorname{Tr}\left(\left.\operatorname{Frob}_{v}\right|_{V_{\nu}(\lambda)}\right)$ is therefore constant on the unique $W_{0}$-orbit on $(W \nu)^{\mathrm{Frob}_{v}}$. This completes the proof.

Proof of part (ii). We are now ready for the proof of part (ii) of the Theorem of §7.2.

Any $\hat{G}$-invariant regular function on $\hat{G} \rtimes$ Frob $_{v}$ gives by restriction a regular function on $\hat{T} \rtimes \operatorname{Frob}_{v}$. As $t \operatorname{Frob}_{v}$ and $t\left(t^{\prime}\right)^{\operatorname{Frob}_{v}}\left(t^{\prime}\right)^{-1} \operatorname{Frob}_{v}$ are conjugate by $t^{\prime}$, this restricted function descends to a regular function on the Frob ${ }_{v}$-coinvariants on $\hat{T}_{v}$, that is to say, it descends to $\hat{A}_{v}$. By the Proposition of $\S 7.1$, this function is $W_{0, v}$-invariant as well. After the Lemma, it only remains to check that any $\hat{G}$-invariant regular function on $\hat{G} \rtimes \operatorname{Frob}_{v}$ that induces the zero function on $\hat{A}_{v}$ (and thus on $\hat{T}$ ), is zero.

The image of the action map $\alpha: \hat{G} \times\left(\hat{T} \rtimes\right.$ Frob $\left._{v}\right) \rightarrow \hat{G} \rtimes$ Frob $_{v}$ is of dimension $\operatorname{dim}(\hat{G})+\operatorname{dim}(\hat{T})-\operatorname{dim}(M)$, where $M$ is the set-wise stabilizer of $\hat{T} \rtimes \operatorname{Frob}_{v}$ in $\hat{G} \rtimes \operatorname{Frob}_{v}$. A Lie algebra computation shows that the identity component of $M$ is $\hat{T}$; consequently $\alpha$ is dominant. This completes the proof.

7.4. Local pseudoroots. We now address the "mismatch" between (7.2.1) and (7.2.3) of Theorem 7.2. After a suitable choice, we can identify the $*$-action and the usual action of $W_{0, v}$ on $\hat{A}_{v}$, thus obtaining the usual statement of the Satake isomorphism. A more intrinsic approach is described in Theorem 7.9 but, for most of this paper, we will follow the ad hoc approach outlined below, because it is enough for our purposes and much closer to the literature.

With notation as in the Theorem, a pseudoroot at $v$ is a fixed point of $\left(W_{0, v}, *\right)$ on $\hat{A}_{v}$ whose square is normalized in a natural way. More precisely, a pseudoroot is a choice of element $\alpha_{0} \in \hat{A}_{v}$ such that

(a) $\alpha_{0}^{2}=\Sigma_{G}^{*}\left(q_{v}\right)$

(b) $\alpha_{0}$ is invariant under the $*$-action of $W_{0, v}$ (see (7.2.1)).

Such always exist: If we choose a square root $\sqrt{q_{v}} \in k^{*}$ we could take $\alpha_{0}=\Sigma_{G}^{*}\left(\sqrt{q_{v}}\right)$. If $\Sigma_{G}^{*}$ is divisible by two, then $\sqrt{\Sigma_{G}^{*}}\left(q_{v}\right)$ gives a particularly natural choice.

The rule $a \mapsto a \alpha_{0}$ defines an isomorphism

$$
\hat{A}_{v} /\left(W_{0, v}, \text { usual action }\right) \longrightarrow \hat{A}_{v} /\left(W_{0, v}, \text { twisted action }\right)
$$

Thus, by composing (7.2.2), pullback under (7.4.1), and (7.2.3), we arrive at an identification

$$
\text { characters of } \mathscr{H}\left(G_{v}, K_{v}\right) \simeq \hat{G} \rtimes \operatorname{Frob}_{v} / / \hat{G}
$$

which we refer to, in short, as the Satake isomorphism. In particular, having fixed a pseudoroot, each element of $\hat{G}(k)$ gives a character $\mathscr{H}\left(G_{v}, K_{v}\right) \rightarrow k$, and two elements $g_{1}, g_{2}$ give the same character precisely when $g_{1} \cdot$ Frob, $g_{2}$. Frob have the same projection to ${ }^{L} \hat{G} / / \hat{G}$. 
There are two other important equivalent ways to think of pseudroots, via the identifications of $\S 2.9$ :

$\hat{A}_{v} \simeq$ conj. classes of splittings ${ }^{L} \hat{T}_{v} \rightarrow\left\langle\right.$ Frob $\left._{v}\right\rangle \simeq$ unramified characters of $T_{v}$

(a) As "square roots of the modular character" of $B_{v}$ :

A pseudoroot gives rise via (7.4.3) to an unramified character of $T_{v}$, thus also an unramified character of $B_{v}$. In this way, pseudoroots are identified with certain preferred square roots of the modular character for $B_{v}$ :

Let $v$ be a good place. By proceeding as in (7.2.4) but using the natural map $q_{v}^{\mathbf{Z}} \rightarrow k^{*}$ instead of the inclusion $q_{v}^{\mathbf{Z}} \subset \mathbf{R}$ we get the " $k$-valued modular character"

$$
\delta: B_{v} \rightarrow F_{v}^{*} \stackrel{|\cdot|_{v}}{\longrightarrow} q_{v}^{\mathbf{Z}} \rightarrow k^{*}
$$

or $\delta=\left|\Sigma_{G}\right|_{v}$ for short. Then a local pseudroot for $\mathbf{G}$ at $v$ corresponds to an unramified character $\delta^{1 / 2}: B_{v} \rightarrow k^{*}$ that squares to $\delta$, and that obeys

$$
\left(\delta^{1 / 2}\right)^{2}=\delta \quad \frac{w \delta^{1 / 2}}{\delta^{1 / 2}}=\left|\sqrt{\frac{w \Sigma_{G}}{\Sigma_{G}}}\right|_{v} \text { for } w \in W_{0, v}
$$

(b) A pseudoroot is uniquely determined by the associated splitting $\rho:\left\langle\operatorname{Frob}_{v}\right\rangle \rightarrow$ ${ }^{L} \hat{T}_{v}$; this splitting sends Frob $_{v}$ to $\widetilde{\alpha_{0}} \cdot$ Frob $_{v}$, where $\widetilde{\alpha_{0}} \in \hat{T}$ is a lift of $\alpha_{0} \in \hat{A}_{v}$.

We will often simply say "let $\rho:\left\langle\operatorname{Frob}_{v}\right\rangle \rightarrow{ }^{L} \hat{T}_{v}$ be a pseudoroot", meaning that $\rho\left(\operatorname{Frob}_{v}\right) \cdot \operatorname{Frob}_{v}^{-1}$ is a pseudoroot in the above sense.

It will also be convenient to use the symbol $\rho^{-}$for the parameter of the inverse pseudoroot, i.e.

$$
\rho^{-}: \operatorname{Frob}_{v} \mapsto \widetilde{\alpha_{0}^{-1}} \operatorname{Frob}_{v} .
$$

i.e. $\rho^{-}$parameterizes a square root of the negative modular character for $B_{v}$.

7.5. Global pseudoroots and canonical pseudoroots. We define a global pseudoroot to be a $k^{*}$-valued idele class character of $\mathbf{T}_{G}^{c a n}$ (the canonical $F$-torus of $\mathbf{G}, \S 2.4$ ) which restricts to a local pseudoroot at almost every good place $v$ - that is to say, the associated character of a Borel subgroup $B_{v} \subset \mathbf{G}\left(F_{v}\right)$, via $B_{v} \rightarrow \mathbf{T}_{G}^{\text {can }}\left(F_{v}\right)$, is a pseudoroot in the sense of the discussion around (7.4.4).

At least in our cases (and presumably always, by a global Langlands for tori with $k^{*}$ coefficients, but we did not verify the validity of this) a global pseudoroot yields a conjugacy class of sections $\rho_{G}$ of ${ }^{L} \hat{T} \rightarrow \Gamma_{F}$ with the property that for almost every $v$, the element $\rho_{G}\left(\operatorname{Frob}_{v}\right) \operatorname{Frob}_{v}^{-1} \in \hat{T}(k)$ projects to an element of $\hat{A}_{v}$ satisfying conditions (a) and (b) of $\S 7.4$.

There are two situations when there is a canonical choice of global pseudoroot:

(a) When the characteristic of $k$ is two, where the trivial character is the canonical choice - equivalently, the trivial splitting where $\rho_{G}\left(\operatorname{Frob}_{v}\right)=$ Frob $_{v}$;

(b) When the half-sum of positive roots $\frac{\Sigma_{G}}{2}: \mathbf{T}_{G}^{\mathrm{can}} \rightarrow \mathbb{G}_{m}$ exists in the character lattice for G. Then we pull back the "cyclotomic" idele class character of $\mathbb{G}_{m}(\mathbf{A}) \rightarrow k^{*}$ via $\frac{\Sigma_{G}}{2}$. (The "cyclotomic" idele class character corresponds to the Hecke character that sends a prime-to- $p$ ideal to its norm in $k^{*}$.) The associated splitting is

$$
\rho_{G}: \gamma \mapsto \frac{\Sigma_{G}^{*}}{2}(\operatorname{cyclo}(\gamma)) \rtimes \gamma,
$$


where cyclo: $\Gamma_{F} \rightarrow \mathbf{F}_{p}^{*}$ is the action of $\Gamma_{F}$ on $p$ th roots of unity in $\bar{F}$.

When both these apply, these canonical choices agree: the half-sum of positive roots determines the trivial character. We shall simply say "there is a canonical pseudoroot" in these cases.

Our focus will be on cases where there is a canonical pseudoroot for both $\mathbf{G}$ and $\mathbf{H}$, but in general there need not be any global pseudoroot at all, e.g. §7.8.

7.6. Parabolic induction and Satake parameters. The isomorphism of (7.2.3) induces a bijection between maximal ideals, i.e. a bijection between semisimple twisted conjugacy classes in $\hat{G}(k)$ and characters of the Hecke algebra. To clarify the role of pseudoroots, let us give an explicit formula for this bijection.

Let $\theta$ be an unramified character of $T_{v}$. We can form the unnormalized parabolic induction $\mathrm{J}_{B}^{G}(\theta)$. This is the submodule of $C_{c}^{\infty}\left(G_{v} ; k\right)$ given by those $s: G_{v} \rightarrow k$ that obey $s(b g)=\theta(b) s(g)$ for $b \in B_{v}$. The $K_{v}$-invariant subspace is a one-dimensional $k$ vector space (because $G_{v}=B_{v} K_{v}$ ), generated by the vector $v^{0}$ whose restriction to $K_{v}$ is identically 1 . For $h \in \mathscr{H}\left(G_{v}, K_{v}\right)$ we have ${ }^{4}$

$$
h v^{0}=\left\langle\mathcal{S}^{*} h, \theta\right\rangle \cdot v^{0}
$$

with $\mathcal{S}^{*}$ as in (7.2.5). In other words, the Hecke algebra acts on the $K_{v}$-fixed vector by the character obtained by pulling back $\theta$ via $\mathcal{S}^{*}$. Let $\chi_{\theta}$ be this character of the Hecke algebra.

Now $\theta$ determines a point $a_{\theta} \in \hat{A}_{v}$ as in (7.4.3), and thus a well-defined conjugacy class $C(\theta) \subset \hat{G}(k) \rtimes \operatorname{Frob}_{v} / / \hat{G}$ - the $\hat{G}$-conjugacy class of any element of the form $t_{\theta}$ Frob $_{v}$ where $t_{\theta} \in \hat{T}$ lifts $a_{\theta} \in \hat{A}_{v}$.

Now fix a pseudoroot, which we think of, by the discussion around (7.4.4), as a square root $\delta^{1 / 2}$ of the modular character. The Satake correspondence is then given by

$$
C(\theta) \longleftrightarrow \chi_{\theta \delta^{1 / 2}}
$$

Or, to say a different way: the Hecke character arising from the induction $\mathrm{J}_{B}^{G}(\theta)$ has parameter given by the class of

$$
t_{\theta} \cdot \rho_{G}^{-}\left(\operatorname{Frob}_{v}\right)
$$

inside $\hat{G}(k) \rtimes \operatorname{Frob}_{v} / / \hat{G}$.

For later use, let us examine the situation when we induce from a parabolic that is not minimal. Suppose $P_{v}$ is a parabolic subgroup and $\theta$ an unramified character of $\mathbf{L}^{\mathrm{ab}}$, the abelianized Levi subgroup for the parabolic $P_{v}$, and its Langlands parameter therefore is a twisted conjugacy class in the dual torus $Z(\hat{L})$ (cf. §2.7); let $l_{\theta} \in Z(\hat{L})$ be a representative. Then the Satake parameter of the character of $\mathscr{H}\left(G_{v}, K_{v}\right)$ on $\mathrm{J}_{P}^{G}(\theta)$ is

$$
l_{\theta} \cdot \rho_{G}^{-}\left(\operatorname{Frob}_{v}\right)
$$

Indeed to verify this we just choose a Borel subgroup $B_{v} \subset P_{v}$ and note that $J_{P}^{G}(\theta) \subset$ $J_{B}^{G}(\theta)$, and use the previous formula.

\footnotetext{
${ }^{4}$ Indeed we compute directly $h v^{0}(e)=\int_{n \in N_{v}, a \in A_{v}, k \in K_{v}} h(a n) \theta(a) d n_{v} d a_{v}$; note that the measure on $G_{v}$, normalized so that the measure of $K_{v}$ is 1 , also decomposes [12, §4.1] via $g=a n k$ as $d a \cdot d n \cdot d k$, where the measures on $A_{v}, N_{v}$ are normalized so that the measures of $A_{v} \cap K_{v}, N_{v} \cap K_{v}$ is 1 .
} 
7.7. The $\sigma$-dual homomorphism. In many cases, the transfer of eigenvectors of Theorem 5.8 arises from a homomorphism of $L$-groups we call the " $\sigma$-dual homomorphism." As in $\S 2.5$, let $\hat{G}$ and $\hat{H}$ be the dual groups to $\mathbf{G}, \mathbf{H}$, and let ${ }^{L} \hat{G}$ and ${ }^{L} \hat{H}$ be the $L$-groups to $\mathbf{G}, \mathbf{H}$.

We have defined at a $\sigma$-good place the normalized Brauer map

$$
\text { br : } \mathscr{H}\left(G_{v}, K_{v}\right) \rightarrow \mathscr{H}\left(H_{v}, U_{v}\right) \text {. }
$$

It seems very likely that this arises from an algebraic homomorphism

$$
\psi_{v}:{ }^{L} \hat{H}_{v} \rightarrow{ }^{L} \hat{G}_{v}
$$

covering the identity map on $\left\langle\operatorname{Frob}_{v}\right\rangle$. Indeed such a $\psi_{v}$ induces by Theorem 7.2 a homomorphism of Hecke algebras. The existence of such a $\psi_{v}$ depends, a priori, on the choice of local pseudoroot.

Even better, we can ask for a single homomorphism

$$
{ }^{L} \hat{\psi}:{ }^{L} \hat{H} \rightarrow{ }^{L} \hat{G}
$$

which induces $\psi_{v}$ for almost all places $v$. Again, there is an implicit choice of global pseudoroots.

We will call such an ${ }^{L} \hat{\psi}$ a $\sigma$-dual homomorphism. We will prove that these exist in "most" cases when $\mathbf{G}$ is simply connected and $\mathbf{H}$ semisimple (see Theorem 9.1). In the presence of a $\sigma$-dual homomorphism we may reformulate Theorem 5.8 as a functorial lift:

Theorem. Suppose there is a $\sigma$-dual homomorphism ${ }^{L} \hat{H} \rightarrow{ }^{L} \hat{G}$. If $\rho: \Gamma_{F} \rightarrow{ }^{L} \hat{H}$ is modular for $\mathbf{H}$, then ${ }^{L} \hat{\psi} \circ \rho$ is modular (for $\mathbf{G}$ ).

Here, we say that $\rho: \Gamma_{F} \rightarrow{ }^{L} \hat{G}(k)$ is modular, with respect to a fixed choice of global pseudoroot for $\mathbf{G}$, if there is a level structure $K$ and a class $h \in H^{*}\left([G]_{K}\right)$ such that

For all but finitely many good places $v$, the class $h$ is an eigenvector for the $\mathscr{H}_{v}$-action whose eigenvalue $\chi: \mathscr{H}_{v} \rightarrow k$ coincides with $\rho\left(\right.$ Frob $\left._{v}\right)$ under Satake.

7.8. The $C$-group and the $c$-group. It is possible that there is no $\sigma$-dual homomorphism at all. A simple example is provided by $\mathbf{G}=\mathrm{PGL}_{2}$ over $F=\mathbf{Q}$ and $\sigma$ an inner automorphism of order 3, with fixed points isomorphic to $\mathbf{H}=\mathrm{PSO}\left(x^{2}+3 y^{2}\right)$. In that case, there is no homomorphism of $L$-groups:

$$
\mathbb{G}_{m} \rtimes \Gamma_{\mathbf{Q}} \rightarrow \mathrm{SL}_{2} \times \Gamma_{\mathbf{Q}},
$$

because the image of complex conjugation (considered in $\Gamma_{\mathbf{Q}}$, on the left-hand side) when projected to $\mathrm{SL}_{2}$ must be an order 2 element that normalizes but does not centralize a nontrivial torus, and none such exists.

Deligne has introduced a mild modification of the $L$-group that allows one to bypass the issues of square roots. It is termed by " $C$-group" by the Buzzard and Gee [9]; we will denote it ${ }^{C} \hat{G}$. It is the quotient ${ }^{L} \hat{G} \times \mathbb{G}_{\mathrm{m}}$ by the order 2 element $e:=\left(\Sigma_{G}^{*}(-1),-1\right) \in$ ${ }^{L} \hat{G} \times \mathbb{G}_{\mathrm{m}}$. Here $\Sigma_{G}^{*}$ denotes the cocharacter $k^{*} \rightarrow \hat{T}(k)$ corresponding to the sum of positive roots of $\mathbf{G}$, as in $\$ 7.4$.

Note that $\Sigma_{G}^{*}(-1)$ is always central, since the pairing of the sum of positive coroots with a root is always even. As $\Sigma_{G}^{*}$ is a product of coroot homomorphisms, which extend to $\mathrm{SL}_{2}$, it always takes values in the commutator subgroup of $\hat{G}$. There is a natural projection ${ }^{C} \hat{G} \rightarrow \Gamma \times \mathbb{G}_{\mathrm{m}}$ : if we identify the set ${ }^{C} \hat{G}(k)$ with $\{1, e\}$-cosets of $\hat{G}(k) \times \Gamma \times k^{*}$, it carries the class of $(g, \gamma, c)$ to $\left(\gamma, c^{2}\right)$. 
Even when there is no global pseudoroot, one expects (see [9, Conjecture 5.3.4] for characteristic zero) Hecke eigenclasses in $H^{*}([G])$ to match homomorphisms $\Gamma_{F} \rightarrow{ }^{C} \hat{G}$ whose composition with this projection is $\gamma \mapsto(\gamma$, cyclo $(\gamma))$. One might hope that in such cases there would be a homomorphism of $C$-groups ${ }^{C} \hat{H} \rightarrow{ }^{C} \hat{G}$ over $\Gamma \times \mathbb{G}_{\mathrm{m}}$. But the same example $\left(\mathbf{G}=\mathrm{PGL}_{2}\right.$ over $\mathbf{Q}, \sigma$ of order 3$)$ also contradicts this hope:

The $C$-group for $\mathrm{PGL}_{2}$ identified with the product $\mathrm{GL}_{2} \times \Gamma_{\mathbf{Q}}$ and the $C$-group for $\mathbf{H}$ is $\left(\mathrm{SO}_{2} \rtimes \Gamma_{\mathbf{Q}}\right) \times \mathbb{G}_{\mathrm{m}}$. We seek

$$
{ }^{C} \hat{\psi}:\left(\mathrm{SO}_{2} \rtimes \Gamma_{\mathbf{Q}}\right) \times \mathbb{G}_{\mathrm{m}} \rightarrow \mathrm{GL}_{2} \times \Gamma_{\mathbf{Q}}
$$

where projection to $\mathbb{G}_{\mathrm{m}}$ on the left should correspond to determinant on the right. The projected map $\mathbb{G}_{\mathrm{m}} \rightarrow \mathrm{GL}_{2}$ must be of the form $x \mapsto\left(\begin{array}{cc}x^{n} & 0 \\ 0 & x^{m}\end{array}\right)$ where $n+m=1$; in particular, the centralizer of this image is the diagonal torus in $\mathrm{GL}_{2}$, and so $\mathrm{SO}_{2} \rtimes \Gamma_{\mathbf{Q}}$ must map into the diagonal torus in $\mathrm{GL}_{2}$. But every element of $\mathrm{SO}_{2}$ is a commutator in $\mathrm{SO}_{2} \rtimes \Gamma_{\mathbf{Q}}$, so that ${ }^{C} \hat{\psi}$ must be trivial on $\mathrm{SO}_{2}$.

This problem can be fixed by reducing the size of the $C$-group, retaining only the essential part of the $\mathbb{G}_{\mathrm{m}}$ factor: Suppose that the characteristic of $k$ is not 2 . We replace the $C$-group by the subgroup ${ }^{c} \hat{G}$ with

$$
{ }^{c} \hat{G}:=\frac{\left\{(g, \gamma, c) \in \hat{G}(k) \times \Gamma \times k^{*}: c^{2}=\operatorname{cyclo}(\gamma)\right\}}{\left(\Sigma_{G}(-1), 1,-1\right)}
$$

Observe that this naturally fits in an extension $\hat{G} \rightarrow{ }^{c} \hat{G} \rightarrow \Gamma$; the extension class corresponds to the 2-cocycle that is the image

$$
\text { cyclo } \in H^{1}\left(\Gamma, k^{*}\right) \stackrel{\text { Bockstein }}{\longrightarrow} H^{2}(\Gamma,\{ \pm 1\}) \stackrel{\Sigma_{G}}{\longrightarrow}(\text { extensions } \hat{G} \rightarrow ? \rightarrow \Gamma),
$$

where the last map simply constructs the extension associated to a cocycle in $H^{2}(\Gamma,\{ \pm 1\})$, by using $\Sigma_{G}:\{ \pm 1\} \rightarrow \hat{G}(k)$.

With this notation, there exists a $\sigma$-dual homomorphisms "for $c$-groups" in the case described above: the $c$-group of $\mathrm{PGL}_{2}$ becomes identified with

$$
\left\{\left(g \in \mathrm{GL}_{2}(k), \gamma \in \Gamma\right): \operatorname{det}(g)=\operatorname{cyclo}(\gamma)\right\},
$$

and the $c$-group of the torus $\mathbf{H}$ is simply the semidirect product $\mathrm{SO}_{2} \rtimes \Gamma$. Then

$$
(h, \gamma) \in \mathrm{SO}_{2} \rtimes \gamma \mapsto\left(\iota(h) w^{\chi(\gamma)}, \gamma\right) \in{ }^{c} \hat{G}
$$

defines a $\sigma$-dual morphism; here $\iota: \mathrm{SO}_{2} \rightarrow \mathrm{GL}_{2}$ is the standard inclusion, $w$ is any element of $\mathrm{O}_{2}-\mathrm{SO}_{2}$ and $\chi: \Gamma \rightarrow \mathbf{Z} / 2 \mathbf{Z}$ is the quadratic character associated to $\mathbf{Q}(\sqrt{-3})$.

As in $\$ 2.6$, we can define a variant ${ }^{c} \hat{G}_{v}$ of the $c$-group, using only the discrete cyclic group generated by Frobenius at $v$ rather than the full Galois group of $F_{v}$. This allows us to close this section with the following intrinsic form of the Satake isomorphism:

7.9. Theorem (Satake isomorphism in terms of $c$-groups). Let $G_{v}=\mathbf{G}\left(F_{v}\right)$ be a reductive $v$-adic group and let $K_{v} \subset G_{v}$ be a maximal compact subgroup satisfying the conditions of $\$ 5.2$. Then there is a natural isomorphism

$$
\mathscr{H}\left(G_{v}, K_{v}\right) \stackrel{\text { regular functions on }}{\longrightarrow} \quad \begin{gathered}
\text { preimage of Frob } \text { in }^{c} \hat{G}_{v} / / \hat{G} .
\end{gathered}
$$

Proof. Choose a square root $\sqrt{q}$ of $q$ inside $k^{*}$. This gives, firstly, an isomorphism

$$
{ }^{L} \hat{G}_{v} \stackrel{\sim}{\longrightarrow}{ }^{c} \hat{G}_{v}
$$


given by $g \rtimes \operatorname{Frob}_{v}^{k} \mapsto\left(g,(\sqrt{q})^{k}, \gamma\right)$. It also gives us a pseudoroot, namely,

$$
b \in B_{v} \mapsto(\sqrt{q})^{v(\delta(b))} .
$$

In this way we obtain the isomorphism of (7.9.1) by comparing (7.9.2) with the Satake isomorphism, in the sense previously described. If we change $\sqrt{q}$ by -1 both the identification of (7.9.1), and the Satake isomorphism change by multiplication by $\Sigma_{G}^{*}(-1) \subset$ $\hat{G}(k)$ inside ${ }^{L} \hat{G}$. Thus, (7.9.1) is independent of choice of $\sqrt{q}$.

In these terms, we may precisely formulate a "better" version of our earlier question:

Suppose $\mathbf{G}$ is semisimple and $\mathbf{H}=\mathbf{G}^{\sigma}$ connected. Does there always exist a map ${ }^{c} \hat{H} \rightarrow{ }^{c} \hat{G}$ which induces via Theorem 7.9 for almost every place $v$, the normalized Brauer map br : $\mathscr{H}\left(G_{v}, K_{v}\right) \rightarrow \mathscr{H}\left(H_{v}, U_{v}\right)$ ?

\section{THE SATAKE PARAMETERS OF THE BRAUER HOMOMORPHISM}

8.1. Computing the Brauer homomorphism. Let $\mathbf{G}$ and $\mathbf{H}=\mathbf{G}^{\sigma}$ be as in §5.5. Suppose that $\mathbf{H}$ is connected. Let $v$ be a $\sigma$-good place (\$5.6), let $\gamma \in \Gamma_{F}$ be a Frobenius element at $v$, and choose a local pseudoroot at $v(\S 7.4)$ for both $\mathbf{G}$ and $\mathbf{H}$.

By Theorem 7.2 and (7.4.2), the normalized Brauer homomorphism gives a map

$$
\operatorname{Spec}(\mathrm{br}): \hat{H} \rtimes \gamma / / \hat{H} \rightarrow \hat{G} \rtimes \gamma / / \hat{G}
$$

We may write the domain and codomain of this map as quotients of the maximal tori $\hat{T}_{H} \subset \hat{H}$ and $\hat{T}_{G} \subset \hat{G}$, respectively. In this section we discuss the extent to which this map is covered by a homomorphism $\hat{N}: \hat{T}_{H} \rightarrow \hat{T}_{G}$. The map $\hat{N}$ is not canonically specified - there is a finite set of choices for it, indexed by what we call " $\gamma$-admissible Borel classes" (§8.6). The map $\hat{N}$ is the "dual norm" associated to the admissible Borel class (\$8.9). We will also attach to $\gamma$ a Levi subgroup $\hat{L}_{\gamma} \subset \hat{G}$, called the associated dual Levi (§8.7 and §8.9). With these notations:

Theorem. Let $\hat{N}: \hat{T}_{H} \rightarrow \hat{T}_{G}$ be the dual norm homomorphism attached to a $\gamma$-admissible Borel class, and let $\hat{L}_{\gamma}$ be the associated dual Levi to $\gamma$.

(1) For every $k$-point $x$ of $\hat{H} \rtimes \gamma / / \hat{H}$, there is a $t \in \hat{T}_{H}(k)$ such that $t \rho_{H}^{-}(\gamma) \in{ }^{L} \hat{T}_{H}$ is a representative for $x$, and $\hat{N}(t) \rho_{G}^{-}(\gamma)$ is a representative for the image of $x$ under the map (8.1.1).

(2) Moreover $t$ can be chosen such that $\hat{N}(t)$ lies in the center of $\hat{L}_{\gamma}$.

Note that part (1) of the Theorem does not assert that (8.1.1) can be extended to a commutative square of the form

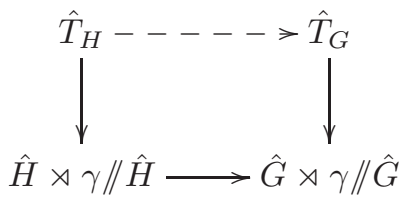

Roughly speaking, the proof of the Theorem goes like this: We compute the effect of Tate cohomology on suitable spherical representations and deduce the computation of the Brauer homomorphism from (6.2.1). In turn, spherical representations are realized in the spaces of sections of suitable line bundles over flag varieties; the main technical step is extending a Borel subgroup of $H_{v}$ to a $\sigma$-stable parabolic subgroup of $G_{v}$, to produce "compatible" flag varieties for $G_{v}$ and $H_{v}$. It is at this step that we need to make choices (the " $\gamma$-admissible Borel classes") from above. 
The arguments in this section resemble the arguments used to prove Theorem 3.3.A of [24], which produces a map similar to (8.1.1), but on the dual side and in characteristic zero. We are grateful to Laurent Clozel for bringing this to our attention; it would be interesting to investigate further.

8.2. Outline of this section. In $\S 8.3-\S 8.9$, we will work with $\mathbf{G}$ and $\mathbf{H}$ over $\bar{F}$. The Galois group makes its mark through its image in $\operatorname{Out}(\mathbf{H})$ and $\operatorname{Out}(\mathbf{G})$. For a fixed element $\gamma \in \operatorname{Out}(\mathbf{H})$, we define certain subgroups $\mathbf{T}_{H}^{\gamma}, \mathbf{L}_{G}(\gamma)$, and a restricted class of Borels $\mathbf{B}_{G}$ and parabolics $\mathbf{P}_{G}$ (the former called " $\gamma$-admissible Borels"). Until $\S 8.10$, all these groups are defined over $\bar{F}$. In $\$ 8.10$ we return to rationality issues. If $\gamma$ is a Frobenius element at $v$, some of these groups (but not $\mathbf{B}_{G}$ ) are defined over $F_{v}$.

8.3. Proposition. Let $\mathbf{T}_{H}$ be a maximal torus in $\mathbf{H}$. Then the centralizer of $\mathbf{T}_{H}$ is a maximal torus in $\mathbf{G}$.

(We repeat: in $\S 8.3-\S 8.9$, all subgroups are to be taken as defined over $\bar{F}$.)

Proof. Let $x \in \mathbf{T}_{H}$ be a regular semisimple element of $\mathbf{H}$. By [32, §8.9], or by [27, Lemma 3.2], we may find a $\sigma$-stable maximal torus and Borel of $\mathbf{G}$ containing $x$, and therefore containing $\mathbf{T}_{H}$. Let $\mathbf{T}_{G} \subset \mathbf{G}$ be such a maximal torus. Then $\mathbf{T}_{H}$ is the identity component of $\mathbf{T}_{G}^{\sigma}=\mathbf{T}_{G} \cap \mathbf{H}$. (In fact since $\mathbf{H}$ is connected and $\mathbf{T}_{H}$ is a maximal torus in $\mathbf{H}$, we actually have $\mathbf{T}_{H}=\mathbf{T}_{G}^{\sigma}$ ). The roots of $\mathbf{T}_{G}$ on the centralizer of $\mathbf{T}_{H}$ are those $\beta \in \Phi\left(\mathbf{T}_{G}, \mathbf{G}\right)$ that are trivial on $\mathbf{T}_{H}$, or equivalently that vanish on the Lie algebra of $\mathbf{T}_{H}$. We will show that there are no such $\beta$, and therefore the centralizer is equal to $\mathbf{T}_{G}$.

The map $\operatorname{Lie}\left(\mathbf{T}_{G}\right)^{*} \rightarrow \operatorname{Lie}\left(\mathbf{T}_{H}\right)^{*}$ identifies the codomain with the $\sigma$-coinvariants of the domain. Thus any $\beta$ that vanishes on $\operatorname{Lie}\left(\mathbf{T}_{H}\right)$ belongs to the image of $1-\sigma$, or equivalently to the kernel of $1+\sigma+\cdots+\sigma^{p-1}$. If $\beta$ is a positive (resp. negative) root, then each $\sigma^{i}(\beta)$ is also positive (resp. negative), and in particular $\beta+\sigma(\beta)+\cdots+\sigma^{p-1}(\beta) \neq 0$. This completes the proof.

8.4. The torus $\left(\mathbf{T}_{H}^{\gamma}\right)^{\circ}$. We continue with the notations of the previous subsection. Let $\mathbf{B}_{H} \subset \mathbf{H}$ be a Borel containing $\mathbf{T}_{H}$. Let $\gamma$ be an outer automorphism of $\mathbf{H}$. (For instance, the image of an element of $\Gamma_{F} \rightarrow \operatorname{Out}(\mathbf{H})$ induced by the $F$-rational structure of $\left.\mathbf{H}\right)$. Then there exists a unique representative for $\gamma$ in $\operatorname{Aut}(\mathbf{H})$ which preserves $\mathbf{T}_{H}$ and $\mathbf{B}_{H}$; we denote this representative also by $\gamma$. Let $\mathbf{T}_{H}^{\gamma}$ denote the group of $\gamma$-fixed points, and $\left(\mathbf{T}_{H}^{\gamma}\right)^{\circ}$ the identity component of $\mathbf{T}_{H}^{\gamma}$.

Proposition. (1) The centralizer of $\left(\mathbf{T}_{H}^{\gamma}\right)^{\circ}$ in $\mathbf{H}$ is $\mathbf{T}_{H}$.

(2) The cone of coweights in $\left(\mathbf{T}_{H}^{\gamma}\right)^{\circ}$ that are positive on $\Phi\left(\mathbf{T}_{H}, \mathbf{B}_{H}\right)$ is "open", i.e. it does not lie in any hyperplane in $X_{*}\left(\left(\mathbf{T}_{H}^{\gamma}\right)^{\circ}\right)$

Proof. The proof of Prop. 8.3, with $\sigma$ replaced by $\gamma$ and $\mathbf{G}$ replaced by $\mathbf{H}$, establishes (1). Let us prove (2). Let $m$ denote the order of $\gamma$ in $\operatorname{Out}(\mathbf{H})$, and consider the operator $\nu$ on $X_{*}\left(\mathbf{T}_{H}\right)$ carrying $\chi$ to $\chi+\gamma \circ \chi+\cdots+\gamma^{m-1} \circ \chi$. After tensoring with $\mathbf{Q}$, the image of $\nu$ coincides with the kernel of $1-\gamma$, in particular the image of $\nu$ is not contained in any hyperplane of $X_{*}\left(\mathbf{T}_{H}^{\gamma}\right)$. Part (2) now follows from the fact that $\nu$ preserves the property of being positive on $\mathbf{B}_{H}$.

8.5. The Levi $\mathbf{L}_{G}(\gamma)$ and its derived group. We let $\mathbf{L}_{G}(\gamma)$ denote the centralizer in $\mathbf{G}$ of the torus $\left(\mathbf{T}_{H}^{\gamma}\right)^{\circ}$. It is a Levi subgroup, and $\Phi\left(\mathbf{T}_{G}, \mathbf{L}_{G}(\gamma)\right) \subset \Phi\left(\mathbf{T}_{G}, \mathbf{G}\right)$ is given by those $\beta: \mathbf{T}_{G} \rightarrow \mathbb{G}_{\mathrm{m}}$ that are trivial on $\left(\mathbf{T}_{H}^{\gamma}\right)^{\circ}$. 
Let us call a cocharacter $\chi: \mathbb{G}_{\mathrm{m}} \rightarrow\left(\mathbf{T}_{H}^{\gamma}\right)^{\circ}$ generic if its centralizer in $\mathbf{G}$ is $\mathbf{L}_{G}(\gamma)$. Each $\delta \in \Phi\left(\mathbf{T}_{G}, \mathbf{G}\right)-\Phi\left(\mathbf{T}_{G}, \mathbf{L}_{G}(\gamma)\right)$ defines an orthogonal hyperplane $H_{\delta} \subset X_{*}\left(\left(\mathbf{T}_{H}^{\gamma}\right)^{\circ}\right)$, and genericity is equivalent to $\chi \notin \bigcup_{\delta} H_{\delta}$.

Note that $\mathbf{L}_{G}(\gamma)$ is $\sigma$-stable, as is $\left[\mathbf{L}_{G}(\gamma), \mathbf{L}_{G}(\gamma)\right]$. We have the following fixed-point computations:

Proposition. The following hold

(1) $\mathbf{L}_{G}(\gamma)^{\sigma}=\mathbf{T}_{H}$.

(2) $\left[\mathbf{L}_{G}(\gamma), \mathbf{L}_{G}(\gamma)\right]^{\sigma}$ is a maximal torus in $\left[\mathbf{L}_{G}(\gamma), \mathbf{L}_{G}(\gamma)\right]$.

Proof. Part (1) follows immediately from part (1) of the proposition of $\S 8.4$. We claim that $\sigma$ induces an inner automorphism of $\left[\mathbf{L}_{G}, \mathbf{L}_{G}\right]$. Since the fixed points of an inner automorphism contain a maximal torus, and $\left[\mathbf{L}_{G}, \mathbf{L}_{G}\right]^{\sigma}$ is contained in a torus by part (1), we can conclude (2).

Let's prove the claim. In fact we will prove that if $\mathfrak{g}$ is a semisimple Lie algebra over $\bar{F}$ and $a$ an automorphism of $\mathfrak{g}$ of prime order $p$, then if $a$ is not inner we cannot have $\mathfrak{g}^{a}$ contained in a Cartan subalgebra. Indeed we may find a pinning $\left(\mathfrak{t}, \mathfrak{b},\left\{e_{\alpha}\right\}_{\alpha \in I}\right)$ of $\mathfrak{g}$ such that $a=\theta \operatorname{ad}_{x}$, where $\theta$ is a pinned automorphism and $x \in \mathfrak{t}$. If $\theta$ is nontrivial then it also has order $p$, and there is a simple root $\alpha$ with $\alpha, \theta(\alpha), \theta^{2}(\alpha) \cdots, \theta^{p-1}(\alpha)$ all distinct.

From the classification of semisimple Lie algebras by Dynkin diagrams, either we may choose $\alpha$ so that the $\theta^{i}(\alpha)$ are all orthogonal, or else $p=2$ and $\mathfrak{g}$ has a factor of the form $\mathfrak{s l}_{3}$ on which $\theta$ acts by transpose-inverse. In the second case, take $\alpha$ to be one of the simple roots of the $\mathfrak{s l}_{3}$-factor. In either case one computes that the elements $e_{\alpha}+a\left(e_{\alpha}\right)+\cdots+$ $a^{p-1}\left(e_{\alpha}\right)$ and $e_{-\alpha}+a\left(e_{-\alpha}\right)+\cdots+a^{p-1}\left(e_{-\alpha}\right)$ do not commute.

8.6. Admissible Borels. We will say that a Borel $\mathbf{B}_{G}$ is $\gamma$-admissible (with respect to $\left.\sigma, \mathbf{T}_{H}, \mathbf{B}_{H}\right)$ if it contains $\mathbf{T}_{G}$ and there exists a cocharacter $\chi: \mathbb{G}_{\mathrm{m}} \rightarrow\left(\mathbf{T}_{H}^{\gamma}\right)^{\circ}$ with the following properties:

(i) $\chi$ is positive for $\mathbf{B}_{H}$ : all nontrivial roots $\beta \in \Phi\left(\mathbf{T}_{H}, \mathbf{B}_{H}\right)$ satisfy $\langle\beta, \chi\rangle>0$

(ii) $\chi$ is nonnegative for $\mathbf{B}_{G}$ : all nontrivial roots $\delta \in \Phi\left(\mathbf{T}_{G}, \mathbf{B}_{G}\right)$ satisfy $\langle\delta, \chi\rangle \geq 0$

(iii) $\chi$ is generic in the sense of $\S 8.5$

A tuple $\left(\mathbf{T}_{H}, \mathbf{B}_{H}, \mathbf{T}_{G}, \mathbf{B}_{G}\right)$, where $\mathbf{T}_{H} \subset \mathbf{B}_{H}, \mathbf{T}_{G}=Z_{\mathbf{G}}\left(\mathbf{T}_{H}\right)$, and $\mathbf{B}_{G}$ is an admissible Borel, will be called a " $\gamma$-admissible Borel tuple." The group $\mathbf{H}(\bar{F})$ acts on $\gamma$-admissible Borel tuples by conjugation - an orbit of this action is called a " $\gamma$ admissible Borel class." Given a homomorphism $\Gamma \rightarrow \operatorname{Out}(\mathbf{H})$, we will say that a tuple $\left(\mathbf{T}_{H}, \mathbf{B}_{H}, \mathbf{T}_{G}, \mathbf{B}_{G}\right)$ is " $\Gamma$-admissible" if it is $\gamma$-admissible for every $\gamma$ in the image.

The significance of admissibility is the following Lemma, which realizes $\mathbf{B}_{H}$ as the $\sigma$-fixed points of a parabolic in $\mathbf{G}$ (cf. discussion after (8.1.2)).

8.7. Lemma. If $\mathbf{B}_{G}$ is a $\gamma$-admissible Borel, then $\mathbf{L}_{G}(\gamma)$ and $\mathbf{B}_{G}$ generate a $\sigma$-stable parabolic subgroup $\mathbf{P}_{G}$ whose $\sigma$-fixed points are $\mathbf{P}_{G}^{\sigma}=\mathbf{B}_{H}$.

Proof. We'll construct $\mathbf{P}=\mathbf{P}_{G}$ by different means, and then show that its $\sigma$-fixed points are $\mathbf{B}_{H}$ and that it is generated by $\mathbf{L}_{G}(\gamma)$ and $\mathbf{B}_{G}$.

Let $\mathfrak{g}$ and $\mathfrak{h}$ denote the $\bar{F}$-linear Lie algebras of $\mathbf{G}$ and $\mathbf{H}$. Similarly let $\mathfrak{t}_{H}$ and $\mathfrak{t}_{G}$ denote the Lie algebras of $\mathbf{T}_{H}$ and $\mathbf{T}_{G}$. We have root space decompositions

$$
\mathfrak{g}=\mathfrak{t}_{G} \oplus \bigoplus_{\beta \in \Phi\left(\mathbf{T}_{G}, \mathbf{G}\right)} \mathfrak{g}_{\beta} \quad \mathfrak{h}=\mathfrak{t}_{H} \oplus \bigoplus_{\delta \in \Phi\left(\mathbf{T}_{H}, \mathbf{H}\right)} \mathfrak{h}_{\delta}
$$

Suppose $\chi$ witnesses the admissibility of $\gamma$, i.e. $\chi$ obeys (i), (ii), and (iii) of $\S 8.6$. 
Let $\mathbf{P} \subset \mathbf{G}$ denote the parabolic subgroup containing $\mathbf{B}_{G}$ whose Lie algebra is the sum of $\mathfrak{t}_{G}$ and those root spaces $\mathfrak{g}_{\beta}$ with $\langle\beta, \chi\rangle \geq 0$. Then $\mathbf{P}$ contains $\mathbf{B}_{G}$.

It is clear that $\mathbf{P}$ is $\sigma$-stable. To see that $\mathbf{P}^{\sigma}=\mathbf{B}_{H}$, note that by assumption (i) for $\chi$, the Lie algebra of $\mathbf{P} \cap \mathbf{H}$ is the Lie algebra of $\mathbf{B}_{H}$, i.e. $(\mathbf{P} \cap \mathbf{H})^{\circ}=\mathbf{B}_{H}$, and $\mathbf{B}_{H}$ is its own normalizer in $\mathbf{H}$.

It remains to show that $\mathbf{P}_{G}$ is generated by $\mathbf{L}_{G}(\gamma)$ and $\mathbf{B}_{G}$. Since both $\mathbf{L}_{G}(\gamma)$ and $\mathbf{B}_{G}$ are connected, the subgroup they generate is connected as well, so this can be checked on Lie algebras, i.e. it is enough to see $\mathfrak{p}=\mathfrak{l}_{G}(\gamma)+\mathfrak{b}_{G}$. We already have $\mathfrak{p} \supset \mathfrak{b}_{G}$, and $\mathfrak{p} \supset \mathfrak{l}_{G}(\gamma)$ follows from

$$
\mathfrak{l}_{G}(\gamma)=\mathfrak{t}_{G} \oplus \bigoplus_{\beta \mid\langle\beta, \chi\rangle=0} \mathfrak{g}_{\beta}
$$

As all three spaces contain $\mathfrak{t}_{G}$, to show that $\mathfrak{p} \subset \mathfrak{l}_{G}(\gamma)+\mathfrak{b}_{G}$ it is enough to prove that a root of $\mathbf{T}_{G}$ on $\mathfrak{p}$ is either a root of $\mathfrak{l}_{G}(\gamma)$, or a root of $\mathfrak{b}_{G}$. Suppose $\mathfrak{g}_{\beta} \subset \mathfrak{p}$ but $\mathfrak{g}_{\beta} \not \subset \mathfrak{l}_{G}(\gamma)$, then $\langle\beta, \chi\rangle>0$. By assumption (ii) for $\chi$, it follows that $\mathfrak{g}_{-\beta}$ is not a root for $\mathbf{B}_{G}$. But $\mathbf{B}_{G}$ is a Borel subgroup: we have $\Phi\left(\mathbf{T}_{G}, \mathbf{G}\right)=\Phi\left(\mathbf{T}_{G}, \mathbf{B}_{G}\right) \amalg\left(-\Phi\left(\mathbf{T}_{G}, \mathbf{B}_{G}\right)\right)$, so $\mathfrak{g}_{-\beta} \not \subset \mathfrak{b}_{G}$ implies $\mathfrak{g}_{\beta} \subset \mathfrak{b}_{G}$. This completes the proof.

Note the proof has shown that $\mathbf{L}_{G}(\gamma)$ is the standard Levi factor of $\mathbf{P}_{G}$, generated by $\mathbf{T}$, the root subgroups for simple roots $\alpha_{i}$ of $\mathbf{B}_{G}$ with $\left\langle\alpha_{i}, \chi\right\rangle=0$, and the roots subgroups for $-\alpha_{i}$.

$\gamma$-admissible Borels always exist, in fact:

\subsection{Lemma. Fix a Borel subgroup and maximal torus $\mathbf{B}_{H} \supset \mathbf{T}_{H}$ in $\mathbf{H}$.}

(1) For any $\gamma \in \operatorname{Out}(\mathbf{H})$, there is a Borel subgroup $\mathbf{B}_{G} \subset \mathbf{G}$ that is $\gamma$-admissible with respect to $\mathbf{B}_{H}, \mathbf{T}_{H}$.

(2) For any group homomorphism $\Gamma \rightarrow \operatorname{Out}(\mathbf{H})$ whose image is cyclic of prime order, there is a Borel subgroup $\mathbf{B}_{G} \subset \mathbf{G}$ that is $\Gamma$-admissible with respect to $\mathbf{B}_{H}, \mathbf{T}_{H}$.

The second assertion of the Lemma is not used in the proof of Theorem $\S 8.1$, but has a further consequence (discussed in $§ 8.15$ ) that will be useful later.

Proof. By part (2) of the Proposition of $\S 8.4$, we may find a cocharacter of $\mathbf{T}_{H}^{\gamma}$ that is positive on $\mathbf{B}_{H}$, i.e. that obeys (i) and (iii) of the conditions for admissibility. Fix such a $\chi$. By "perturbing $\chi$ in $X_{*}\left(\mathbf{T}_{H}\right)$," we may find a Borel $\mathbf{B}_{G}$ obeying (ii). More specifically, let $\epsilon: \mathbb{G}_{\mathrm{m}} \rightarrow \mathbf{T}_{H}$ be any cocharacter that does not vanish any roots of $\Phi\left(\mathbf{T}_{G}, \mathbf{G}\right)$. For $N \in \mathbf{Z}$ sufficiently large, the cocharacter $N \chi+\epsilon$ also does not vanish on any root of $\mathbf{G}$ and therefore determines a positive system in $\Phi\left(\mathbf{T}_{G}, \mathbf{G}\right)$. Let $\mathbf{B}_{G}$ be the corresponding Borel, i.e. with $\delta \in \Phi\left(\mathbf{T}_{G}, \mathbf{B}_{G}\right)$ if and only if $\langle\delta, N \chi+\epsilon\rangle \geq 0$. By taking $N$ sufficiently large, we have $\frac{1}{N}\langle\delta, \epsilon\rangle>-1$ and therefore $\langle\delta, \chi\rangle \geq 0$ for all $\delta \in \Phi\left(\mathbf{T}_{G}, \mathbf{B}_{G}\right)$.

To prove the second assertion it suffices to show that $\mathbf{B}_{G}$ can be chosen simultaneously $\gamma$-admissible and 1-admissible. When $\gamma=1$, we have $\left(\mathbf{T}_{H}^{\gamma}\right)^{\circ}=\mathbf{T}_{H}$, so the cocharacter $N \chi+\epsilon$ also witnesses the 1-admissibility of $\mathbf{B}_{G}$.

8.9. The norm and dual norm homomorphisms. With $\mathbf{T}_{H}$ a maximal torus of $\mathbf{H}$ and $\mathbf{T}_{G}$ its centralizer in $\mathbf{G}$, we define the norm homomorphism $\mathrm{N}: \mathbf{T}_{G} \rightarrow \mathbf{T}_{H}$ by

$$
\mathrm{N}(t)=t \cdot t^{\sigma} \cdots \cdot t^{\sigma^{p-1}}
$$

If we choose $\mathbf{B}_{H} \supset \mathbf{T}_{H}$ and $\mathbf{B}_{G} \supset \mathbf{T}_{G}$, we get an induced map

$$
X^{*}\left(\mathbf{B}_{H}\right) \simeq X^{*}\left(\mathbf{T}_{H}\right) \stackrel{\mathrm{N}^{*}}{\longrightarrow} X^{*}\left(\mathbf{T}_{G}\right) \simeq X^{*}\left(\mathbf{B}_{G}\right)
$$


which in turn induces a map $\hat{T}_{H} \rightarrow \hat{T}_{G}$ which we call the dual norm. Note that (as there is no direct identification of $\mathbf{T}$ with $\mathbf{T}^{\mathrm{can}}$, §2.4) the dual norm depends on $\mathbf{B}_{H}$ and $\mathbf{B}_{G}$.

When $\mathbf{B}_{G}$ is $\gamma$-admissible with respect to $\mathbf{T}_{H}, \mathbf{B}_{H}$, the parabolic $\mathbf{P}_{G}$ of Lemma 8.7 determines (\$2.7) a Levi subgroup $\hat{L}_{\gamma} \subset \hat{G}$ containing $\hat{T}_{G}$. The natural projection $\mathbf{T}_{G} \rightarrow$ $\mathbf{L}_{G}^{\mathrm{ab}}$ dualizes to an inclusion

$$
Z\left(\hat{L}_{\gamma}\right) \hookrightarrow \hat{T}_{G}
$$

8.10. Rationality. Now fix a place $v$ of $F$ at which $\mathbf{H}$ is quasisplit, then we may choose $\mathbf{T}_{H}$ and $\mathbf{B}_{H}$ to be $F_{v}$-rational. The group $\mathbf{T}_{G}:=Z_{\mathbf{G}}\left(\mathbf{T}_{H}\right)$ is also $F_{v}$-rational.

If $\gamma \in \operatorname{Out}(\mathbf{H})$ is the outer automorphism corresponding to the Frobenius, then $\left(\mathbf{T}_{H}^{\gamma}\right)^{\circ}$ is the maximal split subtorus of $\mathbf{T}_{H}$, and its centralizer $\mathbf{L}_{G}(\gamma)$ is $F_{v}$-rational. When $v$ is implicit we write $\mathbf{L}_{G}:=\mathbf{L}_{G}(\gamma)$, for short.

If $\mathbf{B}_{G}$ is any $\gamma$-admissible Borel (relative to $\mathbf{T}_{H}, \mathbf{B}_{H}$ ) then the corresponding parabolic $\mathbf{P}_{G}$ of Lemma 8.7 is $F_{v}$-rational, because any character into the split torus $\left(\mathbf{T}_{H}^{\gamma}\right)^{\circ}$ is automatically $F$-rational and $\mathbf{P}_{G}$ can be defined via the non-negative weight spaces for such a character. Note that we cannot necessarily arrange for $\mathbf{B}_{G}$ itself to be $F_{v}$-rational (nor will we need it), even if $\mathbf{G}$ is quasisplit at $v$. As before we write $\hat{L}_{\gamma}$ for the standard Levi subgroup of $\hat{G}$ associated to the parabolic $\mathbf{P}_{G}$.

8.11. Lemma. Notation as above, $\mathbf{T}_{G} \cap\left[\mathbf{L}_{G}, \mathbf{L}_{G}\right]$ is an anisotropic maximal torus in $\left[\mathbf{L}_{G}, \mathbf{L}_{G}\right]$.

Proof. We appeal to the following basic structural properties of Levi subgroups: $\mathbf{T}_{G} \cap$ $\left[\mathbf{L}_{G}, \mathbf{L}_{G}\right]$ is a maximal torus in $\left[\mathbf{L}_{G}, \mathbf{L}_{G}\right]$, and $\left[\mathbf{L}_{G}, \mathbf{L}_{G}\right] \cap Z\left(\mathbf{L}_{G}\right)$ is finite. As $Z\left(\mathbf{L}_{G}\right)$ contains the maximal split torus $\left(\mathbf{T}_{H}^{\gamma}\right)^{\circ}$, we can prove that $\mathbf{T}_{G} \cap\left[\mathbf{L}_{G}, \mathbf{L}_{G}\right]$ is anisotropic by proving

$$
\mathbf{T}_{H} \cap\left[\mathbf{L}_{G}, \mathbf{L}_{G}\right]=\mathbf{T}_{G} \cap\left[\mathbf{L}_{G}, \mathbf{L}_{G}\right]
$$

The left-to-right containment is obvious, and since $\mathbf{T}_{G}^{\sigma}=\mathbf{T}_{H}$, to show equality it is enough to show that any element of the right-hand group is $\sigma$-fixed. This follows from part (2) of the proposition of $\S 8.5$.

8.12. Lemma. Let $\mathbf{T}_{1}$ and $\mathbf{T}_{2}$ be algebraic tori over $F_{v}$, and suppose that $f: \mathbf{T}_{1} \rightarrow \mathbf{T}_{2}$ has anisotropic kernel. Then precomposition with $f$ induces a surjection

$$
\left\{\text { unramified } k^{*} \text {-valued characters of } \mathbf{T}_{2}\right\} \rightarrow\left\{\text { unramified } k^{*} \text {-valued characters of } \mathbf{T}_{1}\right\}
$$

Proof. Indeed, let $T_{v, i}^{\circ}$ be the maximal compact subgroup of $\mathbf{T}_{i}\left(F_{v}\right)$. Then $f$ induces a map $T_{1, v} / T_{1, v}^{\circ} \rightarrow T_{2, v} / T_{2, v}^{\circ}$ which is injective: Its kernel is a compact subgroup of a free abelian group, thus trivial. Since $k^{*}$ is injective as an abelian group, the result follows.

8.13. Extension of characters. Let us say that a homomorphism $\mathbf{B}_{H}\left(F_{v}\right) \rightarrow k^{*}$ or $\mathbf{P}_{G}\left(F_{v}\right) \rightarrow k^{*}$ is unramified if it factors through an unramified character of $\mathbf{B}_{H}^{\mathrm{ab}}\left(F_{v}\right)=$ $\mathbf{T}_{H}\left(F_{v}\right)$ or $\mathbf{P}_{G}^{\mathrm{ab}}\left(F_{v}\right)=\mathbf{L}_{G}^{\mathrm{ab}}\left(F_{v}\right)$.

Proposition. With notation as in $\$ 8.10$, let $\chi$ be an unramified character of $\mathbf{B}_{H}\left(F_{v}\right)$.

(1) $\chi$ extends to a $\sigma$-invariant unramified character $\chi^{*}$ of $\mathbf{P}_{G}\left(F_{v}\right)$.

(2) $\chi^{*}$ may be chosen in such a way that we may choose representatives $t_{\chi} \in \hat{T}_{H}$ and $t_{\chi^{*}} \in Z\left(\hat{L}_{\gamma}\right) \subset \hat{T}_{G}$ for the Langlands parameters of $\chi$ and $\chi^{*}$, respectively, with

$$
\hat{N}\left(t_{\chi}\right)=\left(t_{\chi^{*}}\right)^{p}
$$

where $\hat{N}$ is the dual norm map associated to the admissible Borel $\mathbf{B}_{G}(\S 8.9)$. 
Proof. Let $\mathbf{K}\left(F_{v}\right)$ be the kernel of the natural projection $\mathbf{T}_{G} \hookrightarrow \mathbf{L}_{G} \rightarrow \mathbf{L}_{G}^{\text {ab }}$. Let $\mathrm{N}(\mathbf{K}) \subset$ $\mathbf{T}_{H}$ be the image of $\mathbf{K}$ under the norm map of §8.9. By Lemma 8.11, $\mathbf{K}$ and $\mathrm{N}(\mathbf{K})$ are anisotropic tori.

The composite $\mathbf{T}_{G} \stackrel{N}{\rightarrow} \mathbf{T}_{H} \rightarrow \mathbf{T}_{H} / \mathrm{N}(\mathbf{K})$ is trivial on $\mathbf{K}$, so it factors through $\mathbf{L}_{G}^{\text {ab }}$. Consider the commutative squares of $F_{v}$-algebraic tori and of associated dual tori
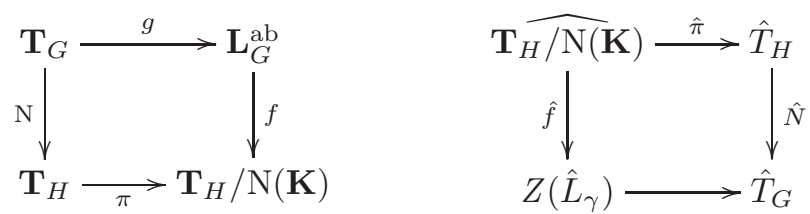

where we use the $\gamma$-admissible Borel class to identify the duals of $\mathbf{T}_{G}, \mathbf{T}_{H}$, and $\mathbf{L}_{G}^{\text {ab }}$ with $\hat{T}_{G}, \hat{T}_{H}$, and $Z\left(\hat{L}_{\gamma}\right)$ as in $\S 8.9$.

Since $\mathrm{N}(\mathbf{K})$ is anisotropic, there is (by Lemma 8.12) an unramified character of $\mathbf{T}_{H} / \mathrm{N}(\mathbf{K})$, call it $\bar{\chi}$, with $\pi^{*} \bar{\chi}=\chi$. Set $\psi=f^{*} \bar{\chi}$, an unramified character of $\mathbf{L}_{G}^{\mathrm{ab}}\left(F_{v}\right)$. Then for $t \in \mathbf{T}_{H}\left(F_{v}\right)$ we have

$$
\psi(g(t))=\bar{\chi}(\pi \circ \mathrm{N}(t))=\chi(t)^{p} .
$$

In other words, $\chi^{*}:=\psi^{1 / p}$ extends $\chi$.

If $t_{\bar{\chi}}$ is a representative for the Langlands parameter of $\bar{\chi}$, then its image $t_{\chi}=\hat{\pi}\left(t_{\bar{\chi}}\right) \in$ $\hat{T}_{H}$ is a representative for the Langlands parameter for $\chi, t_{\psi}=\hat{f}\left(t_{\bar{\chi}}\right) \in Z\left(\hat{L}_{\gamma}\right)$ is a representative for the Langlands parameter of $\psi$, and $t_{\psi}^{1 / p}$ is a representative for the Langlands parameter of $\chi^{*}=\psi^{1 / p}$. (These facts are all readily deduced from (2.9.2)). So (8.13.1) is a consequence of the commutativity of the right-hand square.

8.14. Proof of the Theorem of \$8.1. As in the hypotheses of the Theorem, let $v$ be a place of $F$, let $\gamma \in \Gamma_{F}$ be a Frobenius element at $v$, and let $\mathbf{T}_{H}, \mathbf{B}_{H}, \mathbf{T}_{G}, \mathbf{B}_{G}$ be a $\gamma$ admissible Borel tuple. Let $\mathbf{P}_{G}$ be the corresponding parabolic (Lemma 8.7), and let $\hat{N}$ be the corresponding dual norm (\$8.9).

Fix $\theta \in \hat{H} \rtimes \gamma / / \hat{H}$. We have to find $t \in \hat{T}_{H}$ such that the image of $t \rho_{H}^{-}(\gamma)$ in $\hat{H} \rtimes \gamma / / \hat{H}$ coincides with $\theta$, and is moreover carried by the Brauer homomorphism to the image of $\hat{N}(t) \rho_{G}^{-}(\gamma)$ in $\hat{G} \rtimes \gamma / / \hat{G}$.

We may regard (via Satake (7.4.2)) $\theta$ as a character $\mathscr{H}\left(H_{v}, U_{v}\right) \rightarrow k$. By §7.6, there is an unramified homomorphism $\chi: \mathbf{B}\left(F_{v}\right) \rightarrow k^{*}$ such that $\theta$ is the character by which $\mathscr{H}\left(H_{v}, U_{v}\right)$ acts on the $U_{v}$-invariants of the unnormalized induction $\mathrm{J}_{B_{H}}^{H}(\chi)$. We will show that we may take $t=t_{\chi}$, the element of $\hat{T}_{H}(k)$ corresponding to $\chi$.

Indeed, by $\S 8.13, \chi$ extends to a $\sigma$-invariant character $\chi^{*}$ of $\mathbf{P}_{G}\left(F_{v}\right)$. Restriction from $G_{v}$ to $H_{v}$ gives a surjection (see (3.3.1))

$$
\mathrm{T}^{0}\left(\mathrm{~J}_{P_{G}}^{G}\left(\chi^{*}\right)\right) \rightarrow \mathrm{J}_{B_{H}}^{H}(\chi)
$$

that carries a nonzero $K_{v}$-invariant vector on the left (the function which is identically 1 on $K_{v}$ ) to a nonzero $U_{v}$-invariant vector on the right (the function which is identically 1 on $\left.U_{v}\right)$. Let $\Theta$ be the character by which $\mathscr{H}\left(G_{v}, K_{v}\right)$ acts on $\mathrm{J}_{P_{G}}^{G}\left(\chi^{*}\right)^{K_{v}}$. As $\mathbf{P}_{G}$ and $\chi^{*}$ are $\sigma$-fixed, we may apply (6.2.1) and conclude

$$
\left.\Theta\right|_{\mathscr{H}\left(G_{v}, K_{v}\right)^{\sigma}}=\theta \circ \mathrm{Br}
$$

Thus $(\theta \circ \mathrm{Br})^{p}$ is the character by which $\mathscr{H}\left(G_{v}, K_{v} ; \mathbf{F}_{p}\right)^{\sigma}$ acts on $\mathrm{J}_{P}^{G}\left(\left(\chi^{*}\right)^{p}\right)^{K_{v}}$, by (7.6.1). Recall $\S 4.3$ that br is the linear extension of $\mathrm{Br}^{p}$ from the $\mathbf{F}_{p}$-valued Hecke algebra. Since $\theta \circ$ br extends the $\sigma$-invariant character $(\theta \circ \mathrm{Br})^{p}: \mathscr{H}\left(G_{v}, K_{v} ; \mathbf{F}_{p}\right)^{\sigma} \rightarrow k$ 
and such an extension is unique (§3.4), it follows that $\theta \circ$ br is the character by which $\mathscr{H}\left(G_{v}, K_{v}\right)$ acts on on $\mathrm{J}_{P}^{G}\left(\left(\chi^{*}\right)^{p}\right)^{K_{v}}$. In other words, $\theta \circ$ br $\in \hat{G} \rtimes \gamma / / \hat{G}$ has a representative of the form $t_{\chi^{*}}^{p} \rho_{G}^{-}(\gamma)$, where $t_{\chi^{*}} \in Z(\hat{L})$ is a Langlands parameter for $\chi^{*}$.

The conclusion of the Theorem now follows from (8.13.1)

8.15. A consequence of the Theorem of \$8.1. In this section we show how to use the Theorem of $\S 8.1$ to verify that a candidate homomorphism ${ }^{L} \hat{H} \rightarrow{ }^{L} \hat{G}$ is a $\sigma$-dual homomorphism, in the sense of $\$ 7.7$. The criterion we derive here is not applicable in every case we check - sometimes one has to use $\$ 8.1$ directly — but it is nonetheless very useful.

Suppose that we may find a $\Gamma$-admissible Borel tuple (§8.6), and let $\hat{N}$ be the associated dual norm map (§8.9). Let ${ }^{L} \hat{\psi}:{ }^{L} \hat{H} \rightarrow{ }^{L} \hat{G}$ be a homomorphism over $\Gamma_{F}$, not a priori known to be a $\sigma$-dual homomorphism. If

(a) ${ }^{L} \hat{\psi}$ agrees with $\hat{N}$ on $\hat{T}_{H}$, and

(b) for every $\gamma \in \Gamma_{F}$, the elements ${ }^{L} \hat{\psi}\left(\rho_{H}^{-}(\gamma)\right)$ and $\rho_{G}^{-}(\gamma)$ project to the same element of $\hat{G} \rtimes \gamma / / \hat{L}_{\gamma}$,

then we claim that ${ }^{L} \hat{\psi}$ is a $\sigma$-dual homomorphism.

Whenever (a) holds, (b) is implied by the following condition, which we will often use to verify it:

( $\beta$ ) For every $\gamma \in \Gamma_{F}$, the elements ${ }^{L} \hat{\psi}\left(\rho_{H}^{-}(\gamma)\right)$ and $\rho_{G}^{-}(\gamma)$ of $\hat{G} \rtimes \gamma$ centralize $Z\left(\hat{L}_{\gamma}\right)$ and have the same prime-to- $p$ part, i.e. ${ }^{L} \hat{\psi}\left(\rho_{H}^{-}(\gamma)\right)^{p^{n}}=\rho_{G}^{-}(\gamma)^{p^{n}}$ for some positive integer $n$.

Proof. Indeed, choose a representative $t \rho_{H}^{-}(\gamma)$ for an element of $\hat{H} \rtimes \gamma / / \hat{H}$, as in the statement of Theorem 8.1. Set $\alpha={ }^{L} \hat{\psi}\left(\rho_{H}^{-}(\gamma)\right)$ and $\beta=\rho_{G}^{-}(\gamma)$. We need to see that ${ }^{L} \hat{\psi}\left(t \rho_{H}^{-}(\gamma)\right)=\hat{N}(t) \alpha$ and $\hat{N}(t) \beta$ define the same classes in $\hat{G} \rtimes \gamma / / \hat{G}$, i.e $f(\hat{N}(t) \alpha)=$ $f(\hat{N}(t) \beta)$ for any $\hat{G}$-invariant algebraic function $f$ on $\hat{G} \rtimes \gamma$. But note that $x \mapsto f(\hat{N}(t) x)$ actually defines a regular function on $\hat{G} \rtimes \gamma / / \hat{L}_{\gamma}$, so the claim follows from hypothesis.

To see that $(\beta)$ implies (b): consider the centralizer $\hat{L}^{+}$of $Z\left(\hat{L}_{\gamma}\right)$ inside $\hat{G} \rtimes\langle\gamma\rangle$. Its identity component is $\hat{L}_{\gamma}$. Thus $\alpha, \beta \in \hat{L}^{+}$. For any linear representation $\rho$ of $\hat{L}^{+}$we we have $\operatorname{tr} \rho(\alpha)=\operatorname{tr} \rho(\beta)$ because $\alpha^{p^{n}}=\beta^{p^{n}}$. But the traces of such representations span all $\hat{L}_{\gamma}$-invariant functions on the component of $\hat{L}^{+}$containing $\alpha$ and $\beta$; that was proved in the Lemma of $\$ 7.3$ and concludes the proof.

Finally, we have the following Lemma, which will be useful later:

8.16. Lemma. Fix a $\gamma$-admissible class $\left(\mathbf{T}_{H}, \mathbf{B}_{H} ; \mathbf{T}_{G}, \mathbf{B}_{G}\right)$ for some $\gamma \in \Gamma_{F}$. Let $\hat{N}$ be the dual norm map associated to the Borel class, and let $\hat{T}_{H}^{\gamma}$ denote the $\gamma$-fixed points on $\hat{T}_{H}$. Suppose also that $\mathfrak{h}$ is semisimple and its absolutely simple factors have multiplicity at most 1 . Then

$$
Z_{\hat{G}}\left(\hat{N}\left(\hat{T}_{H}^{\gamma}\right)^{\circ}\right)=\hat{L}_{\gamma} .
$$

Proof. We need to prove that, for a root $\alpha$ of $\mathbf{G}$, it is equivalent for $\alpha$ to be trivial on $\left(\mathbf{T}_{H}^{\gamma}\right)^{\circ}$, and for the associated root $\alpha_{*}^{\vee}$ of $\hat{G}$ to be trivial on $\hat{N}\left(\hat{T}_{H}^{\gamma}\right)^{\circ}$. Here, the subscript $\circ$ denote connected component.

The latter condition is equivalent to

$$
\alpha_{*}^{\vee} \circ \hat{N} \text { is trivial on }\left(\hat{T}_{H}^{\gamma}\right)^{0} .
$$


or equivalently $N \circ \alpha^{\vee}: \mathbb{G}_{m} \rightarrow \mathbf{T}_{H}$ should project trivially to coinvariants $\left(\mathbf{T}_{H}\right)_{\gamma}$, or, what is the same, $N \circ \alpha^{\vee}$ takes image in $(\gamma-1) \mathbf{T}_{H}$.

Write $N_{\gamma}: \mathbf{T}_{H} \rightarrow \mathbf{T}_{H}$ for the " $\gamma$-norm" $\sum_{j=1}^{o} \gamma^{j}$ where $o$ is the order of $\gamma$ acting on $\hat{H}$. Also write $\iota$ for the inclusion of $\mathbf{T}_{H}$ into $\mathbf{T}_{G}$. Thus $(\gamma-1) \mathbf{T}_{H}$ is the connected component of the kernel of $N_{\gamma}$; on the other hand, $\alpha$ is trivial on $\left(\mathbf{T}_{H}^{\gamma}\right)^{\circ}$ if and only if $\alpha \circ \iota N_{\gamma}$ is trivial on $\mathbf{T}_{H}$, i.e. if $\alpha \circ \iota N_{\gamma} N$ is trivial on $\mathbf{T}_{G}$. So we need to check that

$$
\alpha \circ \iota N_{\gamma} N=0 \text { if and only if } \underbrace{\iota}_{\mathbf{T}_{H} \rightarrow \mathbf{T}_{G}} \underbrace{N_{\gamma}}_{\mathbf{T}_{H} \rightarrow \mathbf{T}_{H}} \underbrace{N}_{\mathbf{T}_{G} \rightarrow \mathbf{T}_{H}} \circ \underbrace{\alpha^{\vee}}_{\mathbf{G}_{m} \rightarrow \mathbf{T}_{G}}=0 .
$$

Computing with Lie algebras, we see that it is enough to check that $\iota N_{\gamma} N$, considered as a map $\mathfrak{t}_{G} \rightarrow \mathfrak{t}_{G}$, is self-adjoint with respect to the Killing form on $\mathfrak{g}$. Equip $\mathfrak{t}_{H}$ with the restricted Killing form from $\mathfrak{g}$. Factor this map as

$$
\mathfrak{t}_{G} \stackrel{N}{\rightarrow} \mathfrak{t}_{H} \stackrel{N_{\gamma}}{\rightarrow} \mathfrak{t}_{H} \stackrel{\iota}{\hookrightarrow} \mathfrak{t}_{G}
$$

The adjoint of $N: \mathfrak{t}_{G} \rightarrow \mathfrak{t}_{H}$ with respect to the Killing form is $p \iota$, and the adjoint of $\iota$ is $N / p$.

So it is enough to verify that $N_{\gamma}: \mathfrak{t}_{H} \rightarrow \mathfrak{t}_{H}$ is self-adjoint with respect to the restricted Killing form; and for that it is enough to see that $\gamma$ preserves the restricted Killing form. But on each simple factor of $\mathfrak{h}$, the restricted Killing form from $\mathfrak{g}$ is proportional to the Killing form for $\mathfrak{h}$, and (by virtue of the assumption on $\mathfrak{h}$ ) the action of $\gamma$ preserves the splitting into simple factors.

We could have reached the same conclusion under the following assumption: Split $\mathfrak{h}=\bigoplus \mathfrak{h}_{i}$, where each $\mathfrak{h}_{i}$ is a sum of simple Lie algebras of the same type (and the types of different $\mathfrak{h}_{i}$ are distinct). Then we require that the action of $\gamma$ on each $\mathfrak{h}_{i}$ be induced by an element of $\operatorname{Aut}(\mathfrak{g})$.

\section{Construction of $\sigma$-DUal homomorphisms 1: Preliminaries}

The remainder of this paper is devoted to the proof of the second main theorem, i.e.

9.1. Theorem. Suppose that $\mathbf{G}$ is simply connected and $\mathbf{H}=\mathbf{G}^{\sigma}$ is semisimple, where $\sigma$ is a F-rational automorphism of $\mathbf{G}$ of prime order. Suppose also that $(\operatorname{Lie}(\mathbf{G}), \operatorname{Lie}(\mathbf{H}))$ does not contain a factor isomorphic to $\left(\mathfrak{e}_{6}, \mathfrak{s l}_{3}^{3}\right.$ or $\mathfrak{s l}_{6} \times \mathfrak{s l}_{2}$ or $\left.\mathfrak{s p}_{8}\right)$. Then there exists a $\sigma$-dual homomorphism, with respect to canonical pseudoroots (see Proposition 9.5).

In this somewhat miscellaneous section, we reduce to the case where $\mathbf{G}$ is absolutely almost simple. Part of this reduction involves proving the Conjecture in the important case of cyclic base change. After these reductions, what is left is a finite list of cases, summarized in the Tables at the end of the section.

9.2. Cyclic base change. Let $E / F$ be a cyclic extension, let $\mathbf{H}$ be a simply connected group over $F$, and let $\mathbf{G}=\operatorname{Res}_{E / F}\left(\mathbf{H} \otimes_{F} E\right.$ ) (thus also simply connected). Then a generator $\sigma$ for $\operatorname{Aut}(E / F)$ induces an automorphism of $\mathbf{G}$ fixing $\mathbf{H}$. In this case there is a unique compatible Borel class.

We claim that the canonical "diagonal-restriction" map on $L$-groups ${ }^{L} \hat{H} \rightarrow{ }^{L} \hat{G}$ is a $\sigma$ dual homomorphism. (For generalities on the $L$-group of a restriction of scalars, we refer to $[4, \S 5])$. That follows from $\S 8.1$ and the following facts: with respect to the canonical $\operatorname{map} \iota:{ }^{L} \hat{T}_{H} \rightarrow{ }^{L} \hat{T}_{G}$, the canonical pseudoroots pseudoroots satisfy $\rho_{G}^{-}=\iota \circ \rho_{H}^{-}$and also moreover $\left.\iota\right|_{\hat{T}_{H}}$ is simply the dual norm. 
9.3. Proposition. Suppose that $\mathbf{G}$ has simply connected cover $\mathbf{G}^{\prime}$. Let $\hat{G}^{\prime}$ be the dual group to $\mathbf{G}^{\prime}$. Suppose that canonical pseudoroots exist for $\mathbf{G}, \mathbf{G}^{\prime}, \mathbf{H}, \mathbf{H}^{\prime}=\operatorname{fix}(\sigma)$. If $(\mathbf{G}, \sigma)$ has a $\sigma$-dual homomorphism ${ }^{L} \hat{\psi}:{ }^{L} \hat{H} \rightarrow{ }^{L} \hat{G}$, extending a dual norm map, then so also does $\left(\mathbf{G}^{\prime}, \sigma\right)$.

Note that the converse statement does not hold: if there is a $\sigma$-dual homomorphism for $\widetilde{\mathbf{G}}$, we cannot make any deduction about $\mathbf{G}$.

Proof. Let $\mathbf{H}^{\prime}$ be the fixed points of $\sigma$ on $\mathbf{G}^{\prime}$. The Hecke algebra for $\mathbf{G}^{\prime}$ is identified with a subalgebra of the Hecke algebra for $\mathbf{G}$ and similarly for $\mathbf{H}$. Indeed, let $K_{v} \subset G_{v}$ be a hyperspecial maximal compact subgroup, with preimage $K_{v}^{\prime}$. We may identify $\left(G_{v}^{\prime} / K_{v}^{\prime}\right)$ with a subset of $\left(G_{v} / K_{v}\right)$; moreover, if two elements in $G_{v}^{\prime} / K_{v}^{\prime}$ are in the same $K_{v}$-orbit, they are also in the same $K_{v}$-orbit. Then the identification is given by "extension by zero."

For almost all $v$, the following diagram commutes, where the middle vertical arrows are the inclusions just mentioned

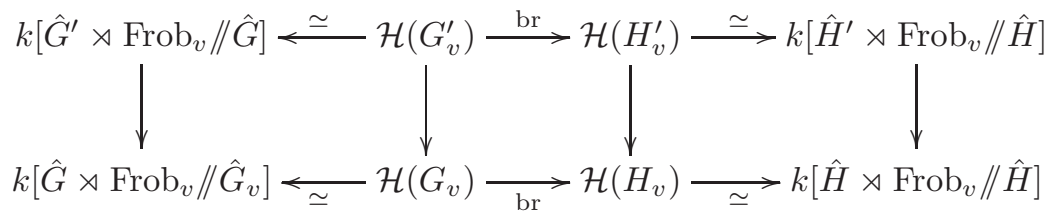

We discuss only commutativity of the central square: It clearly commutes if br is replaced by the un-normalized $\mathrm{Br}$ (vertical arrows are extension by zero, the horizontal arrows are then restriction). The two compositions in the central square are thus seen to be the same on $\mathscr{H}\left(G_{v}^{\prime}, K_{v}^{\prime} ; \mathbf{F}_{p}\right)^{\sigma}$ and by $\S 3.4$ the same on $\mathscr{H}\left(G_{v}^{\prime}, K_{v}^{\prime}\right)$.

It follows from these remarks that, if the map ${ }^{L} \hat{\psi}:{ }^{L} \hat{H} \rightarrow{ }^{L} \hat{G}$ covers a homomorphism ${ }^{L} \hat{\psi}^{\prime}:{ }^{L} \hat{H}^{\prime} \rightarrow{ }^{L} \hat{G}^{\prime}$, then ${ }^{L} \hat{\psi}^{\prime}$ is also a $\sigma$-dual homomorphism. To verify that ${ }^{L} \hat{\psi}$ indeed descends to such a ${ }^{L} \hat{\psi}^{\prime}$, it is enough to check that its projection to ${ }^{L} \hat{G}^{\prime}$ is trivial on the kernel of ${ }^{L} \hat{H} \rightarrow{ }^{L} \hat{H}^{\prime}$, that is, the kernel of $\hat{H} \rightarrow \hat{H}^{\prime}$. (This kernel is regarded as a groupscheme; it may have a single $k$-point.) But that kernel is contained inside $\hat{T}_{H}$; and the dual norm map $\hat{T}_{H} \rightarrow \hat{T}_{G}$ visibly covers a dual norm map $\hat{T}_{H}^{\prime} \rightarrow \hat{T}_{G}^{\prime}$, in particular, is trivial on $\operatorname{ker}\left(\hat{T}_{H} \rightarrow \hat{T}_{H}^{\prime}\right) \simeq \operatorname{ker}\left(\hat{H} \rightarrow \hat{H}^{\prime}\right)$.

9.4. Reduction to the case where $\mathbf{G}$ is absolutely almost simple. Suppose now that $\mathbf{G}$ is simply connected over $F$. We claim that the existence of a $\sigma$-dual homomorphism reduces to the absolutely simple case. Start by noting:

(i) If $(\mathbf{G}, \sigma)=\left(\mathbf{G}_{1}, \sigma_{1}\right) \times\left(\mathbf{G}_{2}, \sigma_{2}\right)$, and the $\mathbf{G}_{i}$ admit $\sigma$-dual homomorphisms, then so does $\mathbf{G}$.

(ii) If $E \supset F$ is an extension field and $(\mathbf{G}, \sigma)=\operatorname{Res}_{E / F}\left(\mathbf{G}^{\prime}, \sigma^{\prime}\right)$, and $\mathbf{G}^{\prime}$ admits a $\sigma$ dual homomorphism, then so does $\mathbf{G}$. (Here the $L$-group of $\mathbf{G}$ is the "induction" of the $L$-group of $\mathbf{G}^{\prime}$, and we just do direct computations with induced groups.)

By a version of $\left[6, \S 6.21\right.$ (ii)], there is an étale $F$-algebra $F^{\prime}$, an $F^{\prime}$-group $\mathbf{G}^{\prime}$ with absolutely almost simple geometric fibers, so that $\mathbf{G}=\operatorname{Res}_{F^{\prime} / F} \mathbf{G}^{\prime}$, and moreover an $F$-automorphism $\tau$ of $F^{\prime}$ and an $\tau$-linear automorphism $\widetilde{\tau}$ of $\mathbf{G}^{\prime}$ which induce $\sigma$.

Now split $\left(F^{\prime}, \tau\right)$ as a product of irreducible factors. There's a corresponding splitting of $\mathbf{G}^{\prime}$, and it is thus enough to consider the case that $\left(F^{\prime}, \tau\right)$ is irreducible, i.e. either $F^{\prime}$ is a field, or $F^{\prime}$ is a sum of $p$ isomorphic fields, permuted by $\tau$; in that case there is also a corresponding decomposition of $\mathbf{G}^{\prime} \simeq \mathbf{G}_{0}^{p}$, the factors being permuted by $p$. The second 
case is easy to check by hand (indeed, it amounts to $\$ 9.2$ in the degenerate case where $\left.E=F^{\oplus p}\right)$.

In the first case ( $F^{\prime}$ a field) we use $\widetilde{\tau}$ to descend $\mathbf{G}^{\prime}$ to a group $\mathbf{G}^{*}$ over the fixed field $F^{*} \subset F^{\prime}$ so that there's an isomorphism

$$
\mathbf{G}^{*} \otimes_{F^{*}} F^{\prime} \simeq \mathbf{G}^{\prime}
$$

and now $\widetilde{\tau}$ on the right-hand side is induced by $\tau \in \operatorname{Aut}\left(F^{\prime} / F^{*}\right)$.

Set $\mathbf{G}^{\prime \prime}=\operatorname{Res}_{F^{\prime} / F^{*}}\left(\mathbf{G}^{*} \otimes_{F^{*}} F^{\prime}\right)$, and let $\sigma^{\prime \prime}$ be the automorphism of $\mathbf{G}^{\prime \prime}$ defined by $\tau \in \operatorname{Aut}\left(F^{\prime} / F^{*}\right)$. Then $(\mathbf{G}, \sigma)=\operatorname{Res}_{F^{\prime} / F}\left(\mathbf{G}^{\prime}, \widetilde{\tau}\right)=\operatorname{Res}_{F^{*} / F}\left(\mathbf{G}^{\prime \prime}, \sigma^{\prime \prime}\right)$. Together with reduction (ii), this is cyclic base change, which we have already discussed (\$9.2)

9.5. Proposition (Canonical pseudoroots). When $\mathbf{G}$ is simply connected and $\mathbf{H}$ is semisimple, both $\mathbf{G}$ and $\mathbf{H}$ have canonical pseudoroots (see $\$ 7.5$ for definition).

Proof. When $p=2$, the trivial section is a canonical pseudoroot and the proposition is trivial, so let us suppose $p>2$. As $\mathbf{G}$ is simply connected, the sum of roots for $\mathbf{G}$ is always divisible by 2 in the weight lattice, but there is something to check for $\mathbf{H}$.

As in $\$ 9.4$, we may reduce to the case where $\mathbf{G}$ is almost simple. We consult the classification given in the tables of $\S 9.6$. Either

(1) $\sigma$ is an order 3 outer automorphism of $\operatorname{Spin}_{8}$ and $\mathbf{H}$ is one of $\mathrm{G}_{2}, \mathrm{PGL}_{3}$.

(2) $\mathbf{G}$ is an exceptional group and $\sigma$ is an inner automorphism of order 3 or 5 .

The outer cases are readily checked. In the inner cases (2), we see from the classification that in every case but one, the sum of positive roots of $\mathbf{H}$ is divisible by 2 even in the adjoint form (because the Lie algebra of $\mathbf{H}$ is a product of copies of $\mathfrak{s l}_{k}$ for $k$ odd.) The exception is the order 3 automorphism of $E_{7}$, whose fixed points have $\mathfrak{h} \simeq \mathfrak{s l}_{3} \times \mathfrak{s l}_{6}$. We verify that $\Sigma_{H}$ is even by hand, using $§ 2.12$.

$$
\Sigma_{H}=96 \alpha_{1}+84 \alpha_{2}+96 \alpha_{3}+160 \alpha_{4}+132 \alpha_{5}+96 \alpha_{6}+52 \alpha_{7}
$$

where $\alpha_{1}, \ldots, \alpha_{7}$ are the simple roots of the simply-connected form of $\mathrm{E}_{7}$ with their Bourbaki numbering.

9.6. Overview of the remainder of the proof. We have verified in $\$ 9.4$ that it suffices to treat the case where $\mathbf{G}$ is almost simple simply connected. In that case, automorphisms of $\mathbf{G} \times{ }_{F} \bar{F}$ whose fixed points are a semisimple group have been classified.

We now recall the classification of prime order automorphisms of simple Lie algebras over an algebraically closed field as a brute list (omitting the several interesting automorphisms of composite order), and refer to [27] for a more subtle discussion. For the outer cases, the "comments" column list the name of the automorphism given in loc. cit., $§ 4$.

Let $\mathfrak{g}$ be a simple Lie algebra over $\bar{F}$, and let $\sigma$ be an automorphism of prime order whose fixed point subalgebra $\mathfrak{g}^{\sigma}$ is semisimple. We tabulate the cases below (the two tables give inner and outer cases) together with references to where Theorem 9.1 is proved, i.e. we verify the existence of a $\sigma$-dual homomorphism for some group $\mathbf{G}$ (not necessarily the simply connected one) with the corresponding Lie algebra. This is enough by Proposition 9.3 .

\section{Construction of $\sigma$-DUAL HOMOMORPhisms 2: INNER CASES}

In this section, which is part of the proof of the second main theorem 9.1, we handle inner cases with $p=3$, except for $\mathrm{E}_{6}$. Throughout this section, suppose $\mathbf{G}$ is a simply connected algebraic group over $F$, and that $\sigma$ is an inner automorphism of $\mathbf{G}$ whose fixed points $\mathbf{H}$ are semisimple. 


\begin{tabular}{|c|c|c|c|c|}
\hline \hline group & fixed subgroup & comments & $p$ & ref \\
\hline $\mathfrak{s l}_{2 n+1}$ & $\mathfrak{s o}_{2 n+1}$ & $\sigma_{0}$ & 2 & 11.5 \\
$\mathfrak{s l}_{2 n}$ & $\mathfrak{s p}_{2 n}$ & $\sigma_{0}$ & 2 & 11.5 \\
$\mathfrak{s l}_{2 n}$ & $\mathfrak{s o}_{2 n}$ & $\sigma_{n}$ & 2 & Th 12.1 \\
$\mathfrak{s o}_{2 n+2}$ & $\mathfrak{s o}_{2 a+1} \times \mathfrak{s o}_{2 b+1}$ & & 2 & Th 12.1 \\
$\mathfrak{s o}_{8}$ & $\mathfrak{g}_{2}$ & $\sigma_{0}$ & 3 & 11.5 \\
$\mathfrak{s o}_{8}$ & $\mathfrak{s l}_{3}$ & $\sigma_{2}$ & 3 & Th 12.1 \\
$\mathfrak{e}_{6}$ & $\mathfrak{f}_{4}$ & $\sigma_{0}$ & 2 & 11.5 \\
$\mathfrak{e}_{6}$ & $\mathfrak{s p}_{8}$ & $\sigma_{4}$ & 2 & \\
\hline \hline
\end{tabular}

\begin{tabular}{|c|c|c|c|c|}
\hline \hline group & fixed subgroup & comments & $p$ & ref \\
\hline $\mathfrak{s o}_{2 n}$ & $\mathfrak{s o}_{2 a} \times \mathfrak{s o}_{2 b}$ & $a, b \geq 2$ & 2 & Th 12.1 \\
$\mathfrak{s o}_{2 n+1}$ & $\mathfrak{s o}_{2 a+1} \times \mathfrak{s o}_{2 b}$ & $b \geq 2$ & 2 & 11.2 \\
$\mathfrak{s p}_{2 n}$ & $\mathfrak{s p}_{2 a} \times \mathfrak{s p}_{2 b}$ & $a, b \geq 1$ & 2 & 11.2 \\
$\mathfrak{g}_{2}$ & $\mathfrak{s l}_{3}$ & & 3 & 10.6 \\
$\mathfrak{g}_{2}$ & $\mathfrak{s o}_{4}$ & & 2 & 11.2 \\
$\mathfrak{f}_{4}$ & $\mathfrak{s l}_{3} \times \mathfrak{s l}_{3}$ & & 3 & 10.6 \\
$\mathfrak{f}_{4}$ & $\mathfrak{s p}_{6} \times \mathfrak{s l}_{2}$ & & 2 & 11.2 \\
$\mathfrak{f}_{4}$ & $\mathfrak{s o}_{9}$ & & 2 & 11.2 \\
$\mathfrak{e}_{6}$ & $\mathfrak{s l}_{3} \times 3$ & & 3 & \\
$\mathfrak{e}_{6}$ & $\mathfrak{s l}_{6} \times \mathfrak{s l}_{2}$ & & 2 & \\
$\mathfrak{e}_{7}$ & $\mathfrak{s l}_{3} \times \mathfrak{s l}_{6}$ & & 3 & 10.6 \\
$\mathfrak{e}_{7}$ & $\mathfrak{s l}_{8}$ & & 2 & 11.2 \\
$\mathfrak{e}_{7}$ & $\mathfrak{s l}_{2} \times \mathfrak{s o}_{12}$ & & 2 & 11.2 \\
$\mathfrak{e}_{8}$ & $\mathfrak{s o}_{16}$ & & 2 & 11.2 \\
$\mathfrak{e}_{8}$ & $\mathfrak{s l}_{9}$ & & 3 & 10.6 \\
$\mathfrak{e}_{8}$ & $\mathfrak{s l}_{5} \times \mathfrak{s l}_{5}$ & & 5 & \\
$\mathfrak{e}_{8}$ & $\mathfrak{s l}_{2} \times \mathfrak{e}_{7}$ & & 2 & 11.2 \\
$\mathfrak{e}_{8}$ & $\mathfrak{s l}_{3} \times \mathfrak{e}_{6}$ & & 3 & 10.6 \\
\hline \hline
\end{tabular}

By a Borel triple for $\mathbf{G}, \mathbf{H}$ we will mean a triple $\left(\mathbf{T}, \mathbf{B}_{H}, \mathbf{B}_{G}\right)$ where $\mathbf{T}$ is a maximal torus in $\mathbf{H} \times_{F} \bar{F}$ and $\mathbf{B}_{H}, \mathbf{B}_{G}$ are Borel subgroups of $\mathbf{H}, \mathbf{G}$ containing $\mathbf{T}$. Note that a Borel triple leads to an identification of canonical tori $\mathbf{T}_{G}^{\mathrm{can}} \simeq \mathbf{T} \simeq \mathbf{T}_{H}^{\mathrm{can}}$, and therefore of dual tori $\hat{T}_{H} \simeq \hat{T}_{G}-$ we denote this identification by $\psi_{1}: \hat{T}_{H} \stackrel{\sim}{\rightarrow} \hat{T}_{G}$. (This differs from the dual norm $\S 8.9$, which in the inner case is a composition of $\psi_{1}$ with a Frobenius endomorphism.)

10.1. Proposition. Let $\left(\mathbf{T}, \mathbf{B}_{H}, \mathbf{B}_{G}\right)$ be a Borel triple and let $\psi_{1}$ denote the corresponding identification $\hat{T}_{H} \stackrel{\sim}{\rightarrow} \hat{T}_{G}$. Then $\psi_{1}$ extends to an injection of dual groups $\psi_{1}^{\prime}: \hat{H} \hookrightarrow \hat{G}$. This extension is moreover unique up to $\hat{T}_{H}$-conjugacy.

As any two maps $\psi_{1}^{\prime}$ are $\hat{T}_{H}$-conjugate, the image of $\hat{H}$ and $\hat{B}_{H}$ under $\psi_{1}^{\prime}$ are welldefined. We will denote them by $\hat{H}_{1}$ and $\psi_{1}^{\prime}\left(\hat{B}_{H}\right)$. If we fix a pinning of $\hat{H}_{1}$ (with $\psi_{1}^{\prime}\left(\hat{B}_{H}\right)$ as the pinned Borel) there is a unique $\psi_{1}^{\prime}$ inside the $\hat{T}_{H}$-conjugacy class of morphisms $\hat{H} \rightarrow \hat{G}$ preserving the pinning.

Proof. By $\S 2.12$ the pair $(\mathbf{G}, \mathbf{H})$ is isomorphic over $\bar{F}$ to a pair as considered in Theorem 2.12. Now Theorem 2.13 establishes the claim for a possibly different choice of Borel subgroups $\mathbf{B}_{H}, \mathbf{B}_{G}$. To obtain $\psi_{1}^{\prime}$, one just applies suitable Weyl group elements.

As to uniqueness, suppose $\psi_{1}^{\prime}, \psi_{1}^{\prime \prime}$ are two possible extensions. Consider any oneparameter unipotent root group $u: \mathbb{G}_{a} \rightarrow \hat{H}$ associated to a simple root. Then $\psi_{1} u, \psi_{1}^{\prime} u$ : $\mathbb{G}_{\mathrm{a}} \rightarrow \hat{G}$ both transform under the same character of $\hat{T}_{G}$, and must differ then by a scaling automorphism of $\mathbb{G}_{a}$. Modifying by a suitable element of $\hat{T}_{H}$, we may suppose that $\psi_{1}, \psi_{1}^{\prime}$ act the same way on all the unipotent root groups in $\hat{H}$ for simple roots, and then they are the same on all of $\hat{H}$.

10.2. Weyl groups and Galois action. Let $\left(\mathbf{T}, \mathbf{B}_{H}, \mathbf{B}_{G}\right)$ be a Borel triple. Let $W_{G}$ and $W_{H}$ be the Weyl groups of $\mathbf{G}$ and $\mathbf{H}$. For each $w \in W_{G}$, the intersection $\operatorname{Ad}(w) \mathbf{B}_{G} \cap \mathbf{H} \subset$ $\mathbf{H}$ is a Borel subgroup of $\mathbf{H}$, and there is therefore a unique $\nu_{w} \in W_{H}$ for which

$$
\operatorname{Ad}(w) \mathbf{B}_{H} \cap \mathbf{H}=\operatorname{Ad}\left(\nu_{w}\right) \mathbf{B}_{H} .
$$

The map $\nu: W_{G} \rightarrow W_{H}$ is left $W_{H}$-equivariant. The preimage $\nu^{-1}(1) \subset W_{G}$ corresponds exactly to those $w$ for which $\operatorname{Ad}(w) \mathbf{B}_{G} \supset \mathbf{B}_{H}$ and is a set of coset representatives for $W_{H} \backslash W_{G}$. We call this set $W_{H \backslash G}$. Then the composite

$$
W_{G} \rightarrow W_{H} \backslash W_{G} \simeq W_{H \backslash G},
$$


will be denoted by $w \mapsto \bar{w}$; explicitly, $\bar{w}=\nu_{w}^{-1} w$.

Now we examine the Galois action. Suppose $\mathbf{T}$ to be chosen $F$-rational. There is now a unique $w(\gamma) \in W_{G}$ so that $\mathbf{B}_{G}^{\gamma}=\operatorname{Ad}(w(\gamma)) \cdot \mathbf{B}_{G}$. Then also $\mathbf{B}_{H}^{\gamma}=\operatorname{Ad}\left(\nu_{w(\gamma)}\right) \mathbf{B}_{H}$. The action of $\gamma$ on $X_{*}\left(\mathbf{B}_{G}\right)$ is given by the composite:

$$
X_{*}\left(\mathbf{B}_{G}\right) \simeq X_{*}\left(\mathbf{B}_{G}^{\gamma}\right) \stackrel{\operatorname{Ad}(w(\gamma))^{-1}}{\longrightarrow} X_{*}\left(\mathbf{B}_{G}\right) .
$$

and now comparing this with the corresponding sequence for $\mathbf{B}_{H}$, we see that

$$
\text { action of } \gamma \text { on } X_{*}\left(\mathbf{B}_{H}\right)=\operatorname{Ad}(\overline{w(\gamma)}) \circ \text { action of } \gamma \text { on } X_{*}\left(\mathbf{B}_{G}\right)
$$

where we emphasize that we have identified $X_{*}\left(\mathbf{B}_{H}\right)$ and $X_{*}\left(\mathbf{B}_{G}\right)$ using $\left(\mathbf{T}, \mathbf{B}_{H}, \mathbf{B}_{G}\right)$. This implies that

$$
\gamma \mapsto \overline{w(\gamma)} \rtimes \gamma \in W_{G} \rtimes \Gamma_{F} \simeq W_{\hat{G}} \rtimes \Gamma_{F}
$$

is a homomorphism, where $\Gamma_{F}$ is acting on the Weyl group of $\mathbf{G}$ according to its outer action on $\mathbf{G}$.

10.3. Proposition. Let $\mathbf{G}$ be a simply-connected semisimple group over $F$ and let $\mathbf{H}$ be the fixed points of an inner automorphism of order $p$ of $\mathbf{G}$. Suppose moreover that

(i) There exists a $\Gamma_{F}$-admissible Borel class in the sense of $\$ 8.6$.

(ii) The center of $\hat{H}$ has only one $k$-point (e.g., it is a group scheme of p-power order). Then there is a homomorphism ${ }^{L} \hat{\psi}:{ }^{L} \hat{H} \rightarrow{ }^{L} \hat{G}$ extending a dual norm map of \$8.9.

Proof. Let $\left(\mathbf{T}, \mathbf{B}_{H} ; \mathbf{T}, \mathbf{B}_{G}\right)$ be a $\Gamma_{F}$-admissible class, and let $\psi_{1}^{\prime}: \hat{H} \rightarrow \hat{G}$ be as in $\S 10.1$, with $\hat{H}_{1}$ the image of $\psi_{1}^{\prime}$; fix a pinning of $\hat{H}_{1}$ which makes $\psi_{1}^{\prime}$ into a pinned map.

Then $\psi_{1}^{\prime}$ extends the identification $\hat{T}_{H} \rightarrow \hat{T}_{G}$ that arises from the choices of Borel subgroups; on the other hand, the dual norm map $\hat{T}_{H} \rightarrow \hat{T}_{G}$ is the $p$ th power of this identification. As $\hat{H}$ is defined in characteristic $p$ and has a natural $\mathbf{F}_{p}$-rational structure, the $p$ th power map on $\hat{T}_{H}$ extends to a $k$-linear Frobenius homomorphism Frob $: \hat{H} \rightarrow \hat{H}$ splitting $\hat{T}_{H}$. So it suffices to show that $\psi_{1}^{\prime} \circ$ Frob $: \hat{H} \rightarrow \hat{G}$ can be extended to $L$-groups.

Let $\alpha$ be a root of $\hat{T}_{H}$ on $\hat{H}$, let $U_{\alpha} \subset \hat{H}$ be the unipotent root group for $\hat{H}$ associated to $\alpha$, and let $\beta=\gamma \alpha$, the image of $\alpha$ under the pinned automorphism of $\hat{H}$ corresponding to some $\gamma \in \Gamma_{F}$. Then inside $\hat{G}$ we have an equality

$$
\operatorname{Ad}(\overline{w(\gamma)} \rtimes \gamma) \cdot \psi_{1}^{\prime} U_{\alpha}=\psi_{1}^{\prime} U_{\beta}
$$

because they are both unipotent subgroups corresponding to the same character of $\hat{T}$ (by (10.2.2)). In particular, the elements $\overline{w(\gamma)} \rtimes \gamma \in W_{\hat{G}} \rtimes \Gamma_{F}$ for $\gamma \in \Gamma_{F}$ normalizes $\hat{H}_{1}$ because it permutes the nontrivial root groups.

Moreover, because of (ii), the element $\overline{w(\gamma)} \rtimes \gamma \in W_{\hat{G}} \rtimes \Gamma_{F}$ can be lifted uniquely to an element $\varpi_{\gamma} \rtimes \gamma \in N_{\hat{G}}(\hat{T})(k) \rtimes \Gamma_{F}$ inducing a pinned automorphism of $\hat{H}_{1}$. (In many cases, we will write down a formula for $\varpi_{\gamma}$ in the course of proving Proposition 10.6). In particular, we have an equality of maps $\hat{H} \rightarrow \hat{H}_{1}$ :

$$
\psi_{1}^{\prime} \circ \gamma=\operatorname{Ad}\left(\varpi_{\gamma} \rtimes \gamma\right) \cdot \psi_{1}^{\prime}
$$

since both sides act the same way on roots and respect pinnings. Now define

$$
{ }^{L} \hat{\psi}: h \rtimes \gamma \in{ }^{L} \hat{H} \mapsto \psi_{1}^{\prime}(\operatorname{Frob}(h))\left(\varpi_{\gamma} \rtimes \gamma\right) \in{ }^{L} \hat{G}
$$

where Frob is the Frobenius. To verify this really is a homomorphism just amounts to checking that $\psi_{1}^{\prime}\left(\operatorname{Frob}\left(\gamma h \gamma^{-1}\right)\right)=\operatorname{Ad}\left(\varpi_{\gamma} \rtimes \gamma\right) \cdot \psi_{1}^{\prime}(\operatorname{Frob}(h))$. But $\operatorname{Frob}\left(\gamma h \gamma^{-1}\right)=$ 
$\gamma \operatorname{Frob}(h) \gamma^{-1}$, because the pinned automorphism of $\hat{H}$ defined by $\Gamma_{F}$ is defined over $\mathbf{F}_{p}$, and so the result follows from (10.3.1).

10.4. The Weyl group element $U$ in the case $p=3$. We now suppose that $p=3$. If $\mathbf{G}$ is almost simple then, examining the classification (see tables in the next section), $\mathbf{G}$ must be of exceptional type EFG if $\mathbf{H}$ is to be semisimple.

Proposition. Suppose that $\mathbf{G}$ is simply connected almost simple not of type $\mathrm{E}_{6}$, that $\sigma$ is inner of order 3 , and that the fixed points $\mathbf{H}$ are semisimple. Let $\left(\mathbf{T}, \mathbf{B}_{H}, \mathbf{B}_{G}\right)$ be a Borel triple. Then

(1) Over $\bar{F}$, the quotient $N_{\mathbf{G}}(\mathbf{H}) / \mathbf{H}$ has precisely two elements.

(2) There is a unique element $U \in W_{G}$ that has a representative that normalizes both $\mathbf{H}$ and $\mathbf{B}_{H}$ and moreover acts by -1 on $Z(\mathbf{H}) / Z(\mathbf{G})$.

Proof. If $\sigma$ is inner and of order 3, then $Z(\mathbf{H}) / Z(\mathbf{G})$ is of order 3, because it is dual to the quotient of the root lattice of $\mathbf{G}$ by the root lattice of $\mathbf{H}$, which by $\$ 2.12(3)$ is of order $p$.

We have already remarked that $\mathbf{G}$ is of exceptional type. If it is not of type $\mathrm{E}_{6}$, then $Z(\mathbf{G})$ has order prime to 3. It follows that if $n \in N_{\mathbf{G}}(\mathbf{H})$ acts trivially on $Z(\mathbf{H}) / Z(\mathbf{G})$, then $n \in \mathbf{H}$, i.e. that the index of $\mathbf{H}$ in $N_{\mathbf{G}}(\mathbf{H})$ has order $\leq 2$. A case by case check of the exceptional groups reveals that the normalizer contains $\mathbf{H}$ with index precisely two. (See [26, Table 10.3]. Note also that when $\mathbf{G}$ is a form of $\mathrm{E}_{6}$ and $\mathbf{H}$ a form of $\mathrm{SL}_{3}^{\times 3} / \Delta \mu_{3}$, $N_{\mathbf{G}}(\mathbf{H}) / \mathbf{H}$ is the symmetric group of order 3.)

To prove (2), note that $\mathbf{H}(\bar{F})$ acts transitively on the set of pairs $\mathbf{B}_{H}^{\prime} \supset \mathbf{T}_{H}^{\prime}$, where $\mathbf{B}_{H}^{\prime} \supset \mathbf{T}_{H}^{\prime}$ are a Borel and maximal torus in $\mathbf{H} \times_{F} \bar{F}$. Thus the nontrivial element of $N_{\mathbf{G}}(\mathbf{H}) / \mathbf{H}$ has a representative $\tilde{U} \in \mathbf{G}(\bar{F})$ that normalizes $\mathbf{H}, \mathbf{B}_{H}$, and $\mathbf{T}$ simultaneously. Any two such must necessarily differ by an element of $\mathbf{T}$, so $\tilde{U}$ determines a unique element of $W_{G}$.

We record, case-by-case, the element $U$ with respect to the Borel triple of $\$ 2.12$. Being of order two in $W_{G}$, it can (see [7, Exercise V.3.3]) be written as a product of commuting root reflections for a finite set of orthogonal positive roots $\left\{r_{1}, \ldots, r_{n}\right\}$ of $\mathbf{G}$. In our cases this can be taken to be the full set of positive (-1)-eigenroots of $U$.

\begin{tabular}{|c|c|c|c|c|}
\hline $\mathbf{G}$ & $\mathfrak{h}$ & $\begin{array}{c}\text { node } \\
\text { of } \\
\Delta_{\mathbf{G}}\end{array}$ & $\left\{r_{1}, \ldots, r_{n}\right\}=$ positive $(-1)$-eigenroots of $U$ & $\Delta_{\mathbf{H}}^{U}$ \\
\hline $\mathrm{G}_{2}$ & $\mathfrak{s l}_{3}$ & 1 & $\alpha_{1}+\alpha_{2}$ & $\varnothing$ \\
\hline $\mathrm{F}_{4}$ & $\mathfrak{s l}_{3} \times \mathfrak{s l}_{3}$ & 2 & $\begin{array}{c}\alpha_{1}+\alpha_{2}+\alpha_{3}+\alpha_{4} \\
\alpha_{1}+\alpha_{2}+2 \alpha_{3}\end{array}$ & $\varnothing$ \\
\hline $\mathrm{E}_{7}$ & $\mathfrak{s l}_{3} \times \mathfrak{s l}_{6}$ & 3 & $\begin{array}{c}\alpha_{1}+\alpha_{2}+\alpha_{3}+2 \alpha_{4}+\alpha_{5} \\
\alpha_{1}+\alpha_{3}+\alpha_{4}+\alpha_{5}+\alpha_{6}+\alpha_{7} \\
\alpha_{1}+\alpha_{2}+\alpha_{3}+\alpha_{4}+\alpha_{5}+\alpha_{6}\end{array}$ & $\alpha_{5}$ \\
\hline $\mathrm{E}_{7}$ & $\mathfrak{s l}_{3} \times \mathfrak{s l}_{6}$ & 5 & $\begin{array}{c}\alpha_{1}+\alpha_{2}+\alpha_{3}+\alpha_{4}+\alpha_{5}+\alpha_{6} \\
\alpha_{2}+\alpha_{3}+2 \alpha_{4}+\alpha_{5}+\alpha_{6}\end{array}$ & $\alpha_{3}$ \\
\hline $\mathrm{E}_{8}$ & $\mathfrak{s l}_{9}$ & 2 & $\begin{array}{r}\alpha_{1}+2 \alpha_{2}+2 \alpha_{3}+3 \alpha_{4}+2 \alpha_{5}+\alpha_{6}+\alpha_{7} \\
\alpha_{1}+\alpha_{2}+2 \alpha_{3}+2 \alpha_{4}+2 \alpha_{5}+\alpha_{6}+\alpha_{7} \\
\alpha_{1}+\alpha_{2}+2 \alpha_{3}+3 \alpha_{4}+2 \alpha_{5}+\alpha_{6}\end{array}$ & $\varnothing$ \\
\hline $\mathrm{E}_{8}$ & $\mathfrak{s l}_{3} \times \mathfrak{e}_{6}$ & 7 & $\begin{array}{r}\alpha_{1}+\alpha_{2}+2 \alpha_{3}+2 \alpha_{4}+\alpha_{5}+\alpha_{6}+\alpha_{7}+\alpha_{8} \\
\alpha_{1}+\alpha_{2}+\alpha_{3}+2 \alpha_{4}+2 \alpha_{5}+\alpha_{6}+\alpha_{7}+\alpha_{8} \\
\alpha_{2}+\alpha_{3}+2 \alpha_{4}+2 \alpha_{5}+2 \alpha_{6}+\alpha_{7}+\alpha_{8}\end{array}$ & $\alpha_{2}, \alpha_{4}$ \\
\hline
\end{tabular}

Note that in this table the two subgroups of $\mathrm{E}_{7}$ are conjugate to each other - we include both for completeness. The $\alpha_{i}$ s are the simple roots of $\mathbf{T}$ on $\mathbf{B}_{G}$ in their Bourbaki numberings [7, Plates I-IX]. The outer action of $U$ on $\mathbf{H}$ is determined by its fixed points $\Delta_{\mathbf{H}}^{U}$ on $\Delta_{\mathbf{H}}$, which are recorded in the rightmost column. Finally, in all the cases that occur, these orthogonal roots mentioned above are in fact strongly orthogonal, i.e. $r_{i} \pm r_{j}$ are not roots, as can be verified from the table. 
10.5. Lemma. Let $\mathbf{G}$ be an almost simple, simply connected group of exceptional type, but not of type $\mathrm{E}_{6}$. Let $\mathbf{H}$ be the semisimple fixed points of an inner automorphism of order 3 , let $\left(\mathbf{T}, \mathbf{B}_{H}, \mathbf{B}_{G}\right)$ be a Borel triple, and let $U \in W_{G}$ be the corresponding Weyl group element of \$10.4. The following hold:

(1) $U=s_{1} \cdot \ldots s_{k}$, where the $s_{i}$ are commuting reflections associated to a set of strongly orthogonal roots $r_{1}, \ldots, r_{k}$ of $\mathbf{T}$ on $\mathbf{B}_{G}$. The $r_{i}$ are uniquely determined as the positive roots $r$ such that $U r=-r$.

(2) If $\mathbf{B}_{G}$ is $\gamma$-admissible with respect to $\mathbf{B}_{H},{ }^{5}$ where $\gamma$ is the outer automorphism of $\mathbf{H}$ determined by $U$, then the roots $r_{i}$ are simple for the positive system determined by $\mathbf{B}_{G}$.

(3) There is at least one $\Gamma$-admissible (in the sense of (3)) Borel $\mathbf{B}_{G}$ such that if $\beta$ is a simple root of $\mathbf{T}$ on $\mathbf{B}_{H}$ that is fixed by $U$, then $\left\langle\rho_{G}, \beta^{\vee}\right\rangle$ is odd, where $\rho_{G}$ denotes half the sum of the positive roots of $\mathbf{T}$ on $\mathbf{B}_{G}$.

Proof. We already proved (1) (we verified it for a different choice of Borel triple, but the statement is independent of that choice).

Let us verify (2). As $\mathbf{B}_{G}$ is $\gamma$-admissible, we may find $\chi \in X_{*}\left(\mathbf{T}^{\gamma}\right)$ (i.e., orthogonal to each of the $r_{i}$ ) that is positive on simple roots of $\mathbf{B}_{H}$ and nonnegative on simple roots of $\mathbf{B}_{G}$. Also, $\chi$ is generic, in that its centralizer in $\mathbf{G}$ coincides with $\mathbf{T}^{\gamma}$. Write $r_{i}$ as a sum of positive simple roots $\sum s_{i}$; since $\chi\left(r_{i}\right)=0$ each of those simple roots must be orthogonal to $\chi$ and then (by genericity) orthogonal to $\mathbf{T}^{\gamma}$, i.e $r_{i}$ is a sum of simple roots each in the -1 eigenspace of $U$. By our prior discussion, each of these simple roots must actually be one of the $r_{i}$ s, so every $r_{i}$ is in fact simple.

Let us prove (3). From the table above, we see that unless $\mathbf{G}, \mathfrak{h}$ is one of $E_{8}, \mathfrak{s l}_{3} \times \mathfrak{e}_{6}$ or $\mathrm{E}_{7}, \mathfrak{s l}_{3} \times \mathfrak{s l}_{6}$, there are no $U$-fixed elements of $\Delta_{\mathbf{H}}^{U}$ at all and the statement is vacuous. Let us treat the remaining cases:

Case $\mathbf{G}=\mathrm{E}_{8}$. Let $\mathbf{B}_{G}, \mathbf{B}_{H}$ be as in $\S 2.12$. $\mathbf{B}_{G}$ is not admissible with respect to $\mathbf{B}_{H}$, but in the basis $\alpha_{i}$ of simple roots of $\mathbf{T}$ on $\mathbf{B}_{G}$, the Borel whose simple roots are

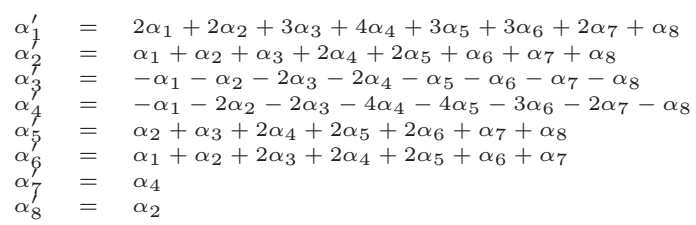

is admissible with respect to $\mathbf{B}_{H}$. The $U$-fixed roots of $\Delta_{H}$ are $\alpha_{2}$ and $\alpha_{4}$, and we compute $\left\langle\rho_{G}, \alpha_{2}^{\vee}\right\rangle=\left\langle\rho_{G}, \alpha_{4}^{\vee}\right\rangle=1$ are both odd.

Case $\mathbf{G}=\mathrm{E}_{7}$. Let $\mathbf{B}_{G}, \mathbf{B}_{H}$ be as in $\S 2.12$. In the basis $\alpha_{i}$ of simple roots of $\mathbf{T}$ on $\mathbf{B}_{G}$, the Borel whose simple roots are

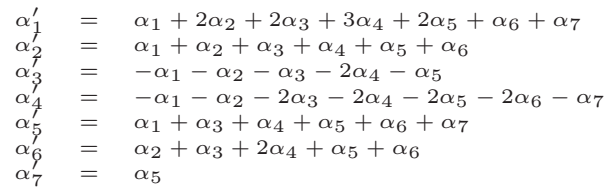

is $\gamma$-admissible. The only $U$-fixed root of $\Delta_{H}$ is $\alpha_{5}$, and $\left\langle\rho_{G}, \alpha_{5}^{\vee}\right\rangle=1$ is odd.

10.6. Proposition. Suppose that $\sigma$ is an inner (F-rational) automorphism of order $p=3$ of a simply connected group $\mathbf{G}$, and over the algebraic closure $(\operatorname{Lie}(\mathbf{G}), \operatorname{Lie}(\mathbf{H}))$ is one of $\left(\mathfrak{g}_{2} \supset \mathfrak{s l}_{3}\right),\left(\mathfrak{f}_{4} \supset \mathfrak{s l}_{3} \times \mathfrak{s l}_{3}\right),\left(\mathfrak{e}_{7} \supset \mathfrak{s l}_{3} \times \mathfrak{s l}_{6}\right),\left(\mathfrak{e}_{8} \supset \mathfrak{s l}_{9}\right)$ or $\left(\mathfrak{e}_{8} \supset \mathfrak{s l}_{3} \times \mathfrak{e}_{6}\right)$. Then there exists a $\sigma$-dual homomorphism.

\footnotetext{
${ }^{5}$ Note this assumption does not apply to the Borel triple used in the table above.
} 
Proof. Note that in all cases listed $\mathbf{G}$ has no nontrivial outer automorphisms. By the discussion of $\S 10.4$ the index of $\mathbf{H}$ in its normalizer is 2. Then (10.2.3) shows that the image of $\Gamma_{F} \rightarrow \operatorname{Out}(\mathbf{H})$ is of order $\leq 2$, so there is a $\Gamma_{F}$-admissible Borel class by Lemma 8.8(2). Fix such a $\Gamma$-admissible Borel class $\left(\mathbf{T}, \mathbf{B}_{H}, \mathbf{T}, \mathbf{B}_{G}\right)$.

As in the first sentence of the proof of 10.3 , if we choose any pinning of $\hat{H}_{1}$ then we can specify ${ }^{L} \hat{\psi}$ in such a way that it preserves pinnings when restricted to $\hat{H}$. We will in fact choose a pinning of $\hat{H}_{1}$ only in in Step 6 below; all the prior steps will be independent of the choice of pinning, because they will depend only on $\psi_{1}^{\prime}$ restricted to $\hat{T}_{H}$.

In each case the center of $\hat{H}$ is a $p$-group $(p=3$ ), so the hypotheses of Proposition 10.3 apply and produce a homomorphism ${ }^{L} \hat{\psi}:{ }^{L} \hat{H} \rightarrow{ }^{L} \hat{G}$ extending the dual norm map associated to the $\Gamma$-admissible class. Explicitly, this is given by

$$
{ }^{L} \hat{\psi}: h \rtimes \gamma \in{ }^{L} \hat{H} \mapsto \psi_{1}^{\prime}(\operatorname{Frob}(h))\left(\varpi_{\gamma} \rtimes \gamma\right) \in{ }^{L} \hat{G}
$$

where Frob is the Frobenius. To prove that ${ }^{L} \hat{\psi}$ is a $\sigma$-dual map, we verify (b) from $\S 8.15$. That is, for each $\gamma \in \Gamma$, we must see that

$$
\rho_{G}^{-}(\gamma) \text { and }{ }^{L} \hat{\psi}\left(\rho_{H}^{-}(\gamma)\right) \text { project to the same element of } \hat{G} \rtimes \gamma / / \hat{L}_{\gamma} \text {, }
$$

where $\hat{L}_{\gamma}$ is defined as in $\S 8.9$, and $\rho_{G}^{-}, \rho_{H}^{-}$are the canonical pseudoroots for $\mathbf{H}, \mathbf{G}$.

Step 1. We claim that $\Gamma_{F} \rightarrow \operatorname{Out}(\mathbf{H})$ is the $\mathbf{F}_{3}^{*}$-valued cyclotomic character. That is $\gamma$ acts nontrivially on $\mathbf{H}$ if and only if $q_{\gamma}=-1 \bmod 3$, where $q_{\gamma} \in \hat{\mathbf{Z}}^{*}$ is the image of $\gamma$ under the cyclotomic character.

Set $\mathbf{S}=\mathbf{T}_{\mathbf{H}}^{\mathrm{can}} / Z(\mathbf{G})$. Now any $\gamma$ fixes $\sigma \in \mathbf{S}(\bar{F})$, where $\sigma$ is the order 3 element such that conjugation by $\sigma$ has $\mathbf{H}$ for fixed points. In other words, "evaluation at $\sigma$," considered in $\operatorname{Hom}\left(X^{*}(\mathbf{S}) / 3, \mu_{3}\right)$ is Galois-invariant. If $\gamma \in \Gamma_{F}$ acts nontrivially on $\mathbf{H}$ its action is the same as that of $U$; in particular, it acts as -1 on $X^{*}(\mathbf{S})$, so it acts as -1 on $\mu_{3}$ too.

Step 2. Let us prove (10.6.2) in the case that $\gamma$ acts trivially on $\mathbf{H}$, i.e. $q_{\gamma}=1$. The element $\overline{w(\gamma)}$, defined in (10.2.2) is trivial here. That means, looking at the way we define ${ }^{L} \hat{\psi}$ from (10.3.2), that $\gamma$ is sent to $\gamma$. It follows that $\rho_{G}^{-}(\gamma)$ and ${ }^{L} \hat{\psi}\left(\rho_{H}^{-}(\gamma)\right)$ coincide already in $\hat{G} \rtimes \gamma$.

Step 3. When $\gamma$ acts nontrivially on $\mathbf{H}$, i.e. $q_{\gamma}=-1$, it acts via the element $U$ of $\S 10.4$. Let us denote the Levi $\hat{L}_{\gamma}$ of $\S 8.9$ by $\hat{L}$. It is obtained from $\hat{T}$ by adjoining the roots $\pm r_{i, *}^{\vee}$, where $r_{i, *}^{\vee}$ are the orthogonal roots of Lemma 10.5.

Step 4. We prove an identity (10.6.3) between the coweights $\Sigma_{G}^{*}$ and $\Sigma_{H}^{*}$, that give rise to the canonical pseudoroots. Let $\Sigma_{G}$ and $\Sigma_{H}$ be the sum of all positive roots of $\mathbf{T}_{G}$ on $\mathbf{B}_{G}$ and $\mathbf{T}_{H}$ on $\mathbf{B}_{H}$ respectively. Let $\Sigma_{G}^{*}$ and $\Sigma_{H}^{*}$ be the corresponding cocharacters of $\hat{T}_{G}$ and $\hat{T}_{H}$ respectively. We identify $\hat{T}_{H}$ and $\hat{T}_{G}$ with each other via the homomorphism $\psi_{1}$ of $\S 10.1$, and:

$$
\Sigma_{G}^{*}-\psi_{1} \circ \Sigma_{H}^{*}=\sum r_{j, *}+\delta
$$

where $\delta \in X_{*}\left(\hat{T}_{G}\right)$ is orthogonal to all $r_{j, *}^{\vee}$, i.e. it is in the center of $\hat{L}$. To prove (10.6.3), it is equivalent to show that $\left\langle\Sigma_{G}^{*}-\psi_{1} \circ \Sigma_{H}^{*}, r_{j, *}^{\vee}\right\rangle=2$ for each $j$, which is a consequence of

(1) $\left\langle\Sigma_{G}, r_{j}^{\vee}\right\rangle=2$ as the $r_{j}$ are simple (Lemma 10.5(3)).

(2) $\left\langle\Sigma_{H}, r_{j}^{\vee}\right\rangle=0$, where we consider $\Sigma_{H}$ as a character of $\mathbf{T}_{G}=\mathbf{T}_{H}$. This is true since $U$ fixes $\Sigma_{H}$ and acts on $r_{j}^{\vee}$ by -1

Step 5. Let $\hat{U} \in W_{\hat{G}}$ correspond to $U \in W_{G}$. We give an explicit formula for an element $\varpi \in N_{\hat{G}}(\hat{T})$ that projects to $\hat{U}$. As $U$ leaves stable the coroots of $\mathbf{H}$, we see that 
$\hat{U}$ leaves stable the roots of $\hat{H}_{1}$, and so it will follow in particular that $\varpi$ normalizes $\hat{H}_{1}$ and induces on it the outer automorphism corresponding to $\gamma$.

Fix a primitive fourth root of unity $\sqrt{-1} \in k$, i.e. a square root of $q_{\gamma}$. By (10.6.3), we have

$$
\Sigma_{G}^{*}(\sqrt{-1})=\psi_{1}^{\prime} \circ \Sigma_{H}^{*}(\sqrt{-1}) \cdot\left(\prod r_{j, *}(\sqrt{-1})\right) \delta(\sqrt{-1})
$$

Let $\iota_{j}: \mathrm{SL}_{2} \rightarrow \hat{L}$ be the coroot homomorphisms corresponding to the $r_{j, *}$, and set

$$
\varpi=\delta(\sqrt{-1}) \prod \iota_{j}(J) \quad \text { where } J:=\left(\begin{array}{rr}
0 & 1 \\
-1 & 0
\end{array}\right) \in \mathrm{SL}_{2}(k)
$$

Both $\varpi$ and $\Sigma_{G}^{*}(\sqrt{-1}) / \psi_{1}^{\prime} \circ \Sigma_{H}^{*}(\sqrt{-1})$ are elements of $\hat{L}$, and by (10.6.3) they are $\hat{L}$-conjugate. In particular, since both $\Sigma_{H}^{*}$ and $\Sigma_{G}^{*}$ are actually divisible by 2 inside the cocharacter group (see Proposition 9.5), $\varpi$ has order 2. Since $\delta(\sqrt{-1}) \in \hat{T}_{G}$, the conjugation action of $\varpi$ on $X^{*}(\hat{T})$ is the product of the conjugation actions of $\iota_{j}(J)$, each of which induces the root reflection through $r_{j, *}^{\vee}$. In other words, $\varpi$ acts as $U$ on $\hat{T}$.

Step 6. We show that there exists a choice of pinning of $\hat{H}_{1}$ such that $\varpi$ acts as a pinned automorphism on $\hat{H}_{1}$, i.e. for each simple $\operatorname{root} \beta$ of $\hat{T}$ on $\psi_{1}^{\prime}\left(\hat{B}_{H}\right)$, there is a nonzero vector $X_{j} \in \operatorname{Lie}\left(\hat{H}_{1}\right)_{\beta}$, and the $X_{j}$ are permuted by the conjugation action of $\varpi$. As $\varpi$ has prime order two, this is possible if and only if $\varpi$ acts trivially on $\operatorname{Lie}(\hat{G})_{\beta}$ whenever $\beta$ is actually fixed by $\varpi$. Such a $\beta$ corresponds (under $\hat{\psi}_{1}$ ) to a simple root $\alpha$ of $\hat{H}$ that is $\gamma$-fixed. Consulting the table of $\S 10.4$, for $\mathrm{G}_{2}, \mathrm{~F}_{4}$, and $\left(\mathrm{E}_{8}, \mathfrak{s l}_{9}\right)$, there is nothing to prove since there are no $U$-fixed simple roots. The remaining two cases are simply-laced, in which case the $\beta$-root group commutes with each $\iota_{j}\left(\mathrm{SL}_{2}\right)$, because $\beta$ is orthogonal (therefore strongly orthogonal in these simply laced cases) to $r_{j}^{\vee, *}$ inside the root system of $\hat{G}$ :

$$
\left\langle\beta, r_{j}^{*}\right\rangle=\left\langle\beta^{*}, r_{j}\right\rangle=0
$$

where $\beta^{*}$ is the associated coroot for $\hat{G}$ - the last because $U$ fixes $\beta^{*}$ and negates $r_{j}$.

Finally, we must check that the $\beta$-root group commutes with $\delta(\sqrt{-1})$, or equivalently that $\langle\delta, \beta\rangle$ is divisible by 4 . From (10.6.3), and the fact that $\left\langle\Sigma_{H}^{*}, \beta\right\rangle=2$ and $\left\langle r_{j}^{*}, \beta\right\rangle=0$, this is equivalent to showing that $\left\langle\frac{1}{2} \Sigma_{G}^{*}, \beta\right\rangle$ is odd, which is part of Lemma 10.5(4).

Step 7. Finally we verify (10.6.2) when $q_{\gamma}=-1$. Note that, e.g., $\rho^{-}(\gamma)=\Sigma_{G}^{*}(\sqrt{-1})$ in this case. Referring to (10.6.1) we must show that

$$
\psi_{1}^{\prime} \Sigma_{H}^{*}(\operatorname{Frob}(\sqrt{-1})) \varpi \gamma=\psi_{1}^{\prime} \circ \Sigma_{H}^{*}(\sqrt{-1}) \varpi \rtimes \gamma \quad \text { and } \quad \Sigma_{G}^{*}(\sqrt{-1}) \rtimes \gamma
$$

of ${ }^{L} \hat{G}$ are conjugate under $\hat{L}_{\gamma}$. Because $\mathbf{G}$ is inner, it is sufficient to show that $\Sigma_{G}^{*}(\sqrt{-1})$ and $\psi_{1}^{\prime} \Sigma_{H}^{*}(\sqrt{-1}) \varpi$ are $\hat{L}$-conjugate. This follows from the remarks of Step 5. (note that, as $\left\langle\Sigma_{H}, r_{j}^{\vee}\right\rangle=0$, the map $\psi_{1}^{\prime} \circ \Sigma_{H}^{*}$ takes values in the center of $\hat{L}_{\gamma}$ ).

\section{CONSTRUCTION OF $\sigma$-DUAL HOMOMORPHISMS 3: EXCEPTIONAL ISOGENIES}

In this section we treat two large "clusters" of cases. One of these involves heavily the "exceptional isogenies" of semisimple groups that exist only in special characteristic. The remaining cases are checked individually, and they are discussed in the final section.

11.1. Remark on twisting. We will use repeatedly the following remark: Suppose that $(\mathbf{G}, \sigma, \mathbf{H}=\operatorname{fix}(\sigma))$ admits a form $\left(\mathbf{G}^{\prime}, \sigma^{\prime}, \mathbf{H}^{\prime}=\operatorname{fix}\left(\sigma^{\prime}\right)\right)$ defined over $F$ with $\mathbf{G}^{\prime}$ and $\mathbf{H}^{\prime}$ both $F$-split groups. Then $\mathbf{G}, \sigma$ is obtained by twisting from $\left(\mathbf{G}^{\prime}, \sigma^{\prime}\right)$ by an element of

$$
H^{1}\left(\Gamma, \operatorname{Aut}\left(\mathbf{G}^{\prime}, \sigma^{\prime}, \mathbf{H}^{\prime}\right)\right) \text {. }
$$


This group of automorphisms is precisely the centralizer $Z_{\mathrm{Aut}\left(\mathbf{G}^{\prime}\right)}\left(\sigma^{\prime}\right)$ of $\sigma^{\prime}$ inside $\operatorname{Aut}\left(\mathbf{G}^{\prime}\right)$.

In particular, the image of $\Gamma \rightarrow \operatorname{Out}(\mathbf{H})$ lies inside the image of $Z_{\mathrm{Aut}_{\left(\mathbf{G}^{\prime}\right)}}\left(\sigma^{\prime}\right)$ in $\operatorname{Out}\left(\mathbf{H}^{\prime}\right) \simeq \operatorname{Out}(\mathbf{H})$ and similarly for the image of $\Gamma \rightarrow \operatorname{Out}(\mathbf{G})$. For example, if $Z_{\text {Aut }\left(\mathbf{G}^{\prime}\right)}\left(\sigma^{\prime}\right)$ is connected, then both $\mathbf{H}$ and $\mathbf{G}$ are necessarily inner.

\subsection{Proposition. Suppose the following conditions hold:}

(i) Out $(\mathbf{G})$ is trivial (so that $\mathbf{G}$ and $\sigma$ are both inner) and $p=2$;

(ii) The image of $\Gamma$ in $\operatorname{Out}(\mathbf{H})$ is of order $\leq 2$; thus we may choose (Lemma 10.3) a $\Gamma$-admissible Borel class.

(iii) $\pi_{1} \mathbf{H}$ is a 2-group.

then ${ }^{L} \hat{\psi}$ as defined by Proposition 10.3 is a $\sigma$-dual homomorphism. These conditions hold when the Lie algebras of $\mathbf{G} \supset \mathbf{H}$ are isomorphic over $\bar{F}$ to a pair from the following list: $\left(\mathfrak{s o}_{2 n+1} \supset \mathfrak{s o}_{2 u+1} \times \mathfrak{s o}_{2 v}\right)$, or $\left(\mathfrak{s p}_{2 n} \supset \mathfrak{s p}_{2 u} \times \mathfrak{s p}_{2 v}\right)$ for $n \geq 2, u, v \geq 1$, and $u+v=n$; or $\left(\mathfrak{g}_{2} \supset \mathfrak{s o}_{4}\right),\left(\mathfrak{f}_{4} \supset \mathfrak{s p}_{6} \times \mathfrak{s l}_{2}\right.$ or $\left.\mathfrak{s o}_{9}\right),\left(\mathfrak{e}_{7} \supset \mathfrak{s l}_{2} \times \mathfrak{s o}_{12}\right.$ or $\left.\mathfrak{s l}_{8}\right),\left(\mathfrak{e}_{8} \supset \mathfrak{s o}_{16}\right.$ or $\left.\mathfrak{s l}_{2} \times \mathfrak{e}_{7}\right)$.

Proof. By inspection all cases have properties (i), (ii); and for (iii) Out(H) itself has at most two elements, except in the first-listed case with $v=4$. But then the image of $Z_{\mathrm{Aut}\left(\operatorname{Spin}_{2 n+1}\right)}(\sigma)=Z_{\mathrm{SO}_{2 n+1}}(\sigma)$ in $\operatorname{Out}(\mathbf{H})$ has order $\leq 2$ (the centralizer has two connected components), and that $\Gamma \rightarrow \operatorname{Out}(\mathbf{H})$ has image of size $\leq 2$ follows from $\S 11.1$.

In all cases, an application of Lemma 10.3 now shows that the dual norm map extends to ${ }^{L} \hat{H} \rightarrow{ }^{L} \hat{G}$. It remains to check $\S 8.15$ (b). Let us show that the stronger condition $\S 8.15(\beta)$ holds. The pseudoroots are trivial, since $p=2$; that is to say $\rho_{H}(\gamma)=\gamma \in{ }^{L} \hat{H}$ and $\rho_{G}(\gamma)=\gamma \in{ }^{L} \hat{G}$. The condition to be checked is that $\gamma$ and ${ }^{L} \hat{\psi}(\gamma)$ both centralize $Z\left(\hat{L}_{\gamma}\right)$, and that

$$
\gamma^{2^{n}}={ }^{L} \hat{\psi}(\gamma)^{2^{n}} \quad \text { for } n \gg 0
$$

Recall that on the left $\gamma$ denotes an element of ${ }^{L} \hat{G}$ and on the right it denotes an element of ${ }^{L} \hat{H}$.

The hypotheses of Lemma 8.16 apply in all cases except $\mathfrak{g}_{2} \supset \mathfrak{s o}_{4}$ : this is clear except in the cases when $\mathfrak{h}$ has a repeated factor i.e. $\left(\mathfrak{s o}_{2 n+1} \supset \mathfrak{s o}_{2 u+1} \times \mathfrak{s o}_{2 v}\right)$ with $v=2$, or $\left(\mathfrak{s p}_{2 n} \supset \mathfrak{s p}_{2 u} \times \mathfrak{s p}_{2 v}\right)$ with $u=v$. In each of these cases, the criterion remarked after the proof of Lemma 8.16 applies. For the case $\mathfrak{g}_{2} \supset \mathfrak{s o}_{4}$, the normalizer of $\mathbf{H}$ in $\mathbf{G}$ is trivial, so by (10.2.2) the conclusion of Lemma 8.16 is trivially true.

As $\mathbf{G}$ is inner, ${ }^{L} \hat{G}=\hat{G} \times \Gamma_{F}$ and $\gamma \in{ }^{L} \hat{G}$ automatically centralizes $Z\left(\hat{L}_{\gamma}\right)$. Meanwhile $\gamma \in{ }^{L} \hat{H}$ centralizes $\hat{T}_{H}^{\gamma}$ by definition, so that ${ }^{L} \hat{\psi}(\gamma)$ centralizes $\hat{N}\left(\hat{T}_{H}^{\gamma}\right)$, the image of $\hat{T}_{H}^{\gamma}$ under the dual norm. Now conjugation by ${ }^{L} \hat{\psi}(\gamma)$ gives an inner automorphism of $\hat{G}$, and according to Lemma 8.16 that inner automorphism is represented by an element of $\hat{L}_{\gamma}$; so ${ }^{L} \hat{\psi}(\gamma)$ centralizes $Z\left(\hat{L}_{\gamma}\right)$ as well.

Since the image of $\Gamma_{F}$ in $\operatorname{Out}(\mathbf{H})$ and $\operatorname{Out}(\mathbf{G})$ both have order at most 2, both $\gamma^{2}$ and ${ }^{L} \hat{\psi}(\gamma)^{2}$ must centralize $\hat{H}_{1}={ }^{L} \hat{\psi}(\hat{H})$, i.e. they must differ by an element of $\hat{G}$ that centralizes the image of $\hat{H}_{1}$. Now, by our explicit construction $(\S 10), \hat{H}_{1} \subset \hat{G}$ is a subgroup of maximal rank. Thus, any element that centralizes the image of $\hat{H}_{1}$ lies inside $\hat{H}_{1}$; but then it lies in the center of $\hat{H}_{1}(k)=\{e\}$ (because $\pi_{1}(\mathbf{H})$ is a 2-group).

11.3. When $\sigma$ is pinned. We now study the case where $(\mathbf{G}, \sigma)$ is isomorphic, over the algebraic closure, to a pinned automorphism of an almost simple simply connected group $\mathbf{G}$, leaving stable a maximal torus and Borel $\mathbf{T} \subset \mathbf{B} \subset \mathbf{G} \times{ }_{F} \bar{F}$. 
Put $\mathbf{T}_{H}:=\mathbf{T}^{\sigma}$ and $\mathbf{B}_{H}:=\mathbf{B}^{\sigma}-$ as $\mathbf{G}$ is simply-connected, they are a maximal torus and Borel subgroup of $\mathbf{H}$. As $\mathbf{G}$ is almost simple, Out $(\mathbf{H})$ is trivial and $\mathbf{B}$ is $\Gamma_{F^{-}}$ admissible (i.e. 1-admissible) with respect to $\mathbf{T}_{H}, \mathbf{B}_{H}$.

For each root $\alpha$ of $\mathbf{G}$, let $\bar{\alpha}$ be its restriction to $\left(\mathbf{T}^{\sigma}\right)^{\circ}$. In general, the roots of $\mathbf{H}$ are in one-to-one correspondence with the " $\sigma$-equivalence classes" of roots of $\mathbf{T}$ on $\mathbf{G}$ - here $\alpha$ and $\beta$ are $\sigma$-equivalent if $\bar{\alpha}=r \bar{\beta}$ for some $r>0$. In particular, the number of roots of $\mathbf{H}$ is the number of distinct rays in $\mathbf{R} \otimes X^{*}\left(\mathbf{T}^{\sigma}\right)$ of the form $\mathbf{R}_{>0} \bar{\alpha}$. For further discussion of this and what follows, we refer to [27, §§3.3-3.4] and [31, p. 177].

Any $\sigma$-equivalence class $a$ is the positive roots of a system of type either $A_{1}^{r}$ or $A_{2}$ ([31, p. 177]). We say accordingly that $a$ (or any $\alpha \in a$ ) is " of type $A_{1}^{r}$ " or "of type $A_{2}$." Type $A_{2}$ occurs only in type $\mathrm{SL}_{2 n+1}$, namely $a=\left\{e_{i}-e_{n+1}, e_{n+1}-e_{i^{*}}, e_{i}-e_{i^{*}}\right\}$ where $i+i^{*}=2 n+2$ and we use the standard realization of the root system $A_{2 n}$. Moreover, if a root is of type $A_{1}^{r}$, then $r$ is the size of the $\sigma$-orbit of $\alpha$.

If $a$ is a $\sigma$-equivalence class of roots, the corresponding root of $\mathbf{T}^{\sigma}$ on $\mathbf{H}$ is $e \bar{\alpha}$ for a unique $e \in \mathbf{Q}_{>0}$; here $\alpha$ is any element of $a$. We set $e_{\alpha}=e$ for any $\alpha \in a$. Also, we write $\theta_{\alpha}$ for the angle between $\alpha$ and $\bar{\alpha}$ with respect to a $W$-invariant inner product on $X^{*}(\mathbf{T}) \otimes \mathbf{R}$. Finally, we let $o_{\alpha}$ to be the order of the $\sigma$-orbit of $\alpha$, so that $o_{\alpha}=1$ or $p$ according to whether $\alpha$ is $\sigma$-fixed or not.

The pinned automorphism $\sigma$ also acts as a pinned automorphism on $\hat{G}$. Let $\hat{G}^{\sigma}$ be the fixed locus with its reduced subscheme structure. If $\alpha_{*}^{\vee}$ is a root of $\hat{T}$ on $\hat{G}$, we let $\overline{\alpha_{*}^{\vee}}$ denote its restriction to $\hat{T}^{\sigma}=\left(\hat{T}^{\sigma}\right)^{\circ}$. In general a positive multiple $f_{\alpha} \overline{\alpha_{*}^{\vee}}$ of $\overline{\alpha_{*}^{\vee}}$ is a root of $\hat{T}^{\sigma}$ on $\hat{G}^{\sigma}$.

In particular $\hat{G}^{\sigma}$ and $\mathbf{H}$ are both semisimple, and have the same rank and the same number of roots - however, they are not always dual groups, but they are isogenous:

11.4. Lemma. Suppose that for all roots $\alpha$ of $\mathbf{T}$ on $\mathbf{G}$,

$$
p e_{\alpha} f_{\alpha} \cos ^{2}\left(\theta_{\alpha}\right) \in\left\{1, p, p^{2}, \ldots\right\}
$$

Then the dual norm defined by $\left(\mathbf{B}_{H}, \mathbf{B}_{G}\right)$ extends to an isogeny $\hat{H} \rightarrow \hat{G}^{\sigma}$.

For example, for pinned triality acting on $\operatorname{Spin}(8)$, the dual norm composes the inclusion $\mathrm{G}_{2} \rightarrow \mathrm{PSO}_{8}$ with an exceptional isogeny $\mathrm{G}_{2} \rightarrow \mathrm{G}_{2}$.

By explicit computation in the type $\mathrm{A}_{2}$ case,

$$
\left(e_{\alpha}, f_{\alpha}, \cos ^{2}\left(\theta_{\alpha}\right)\right)=\left\{\begin{array}{l}
\left(\frac{1}{2} o_{\alpha}, o_{\alpha}, o_{\alpha}^{-2}\right), \text { type } \mathrm{A}_{2}, \\
\left(1,1, o_{\alpha}^{-1}\right) \text { else }
\end{array}\right.
$$

and so the assumption (11.4.1) always applies. Indeed, when $\mathbf{G}$ is not a form of $\mathrm{SL}_{2 n+1}$, this isogeny is a "special isogeny", switching short and long roots from $\hat{H}$ to $\hat{G}^{\sigma}$.

Proof. The norm is a $\sigma$-invariant map $\mathbf{T}_{G}$ to $\mathbf{T}_{H}$, and the dual norm $\hat{N}$ takes $\hat{T}_{H}$ to $\hat{T}_{G}^{\sigma}$, a maximal torus of $\hat{G}^{\sigma}$. We will apply Chevalley's isogeny theorem, which says that $\hat{N}$ extends to an isogeny so long as for each root $\alpha$ of $\hat{G}^{\sigma}$, there is a root $\alpha^{\prime}$ of $\hat{H}$ and a $q_{\alpha} \in\left\{1, p, p^{2}, \ldots\right\}$ such that $\hat{N}$ induces

$$
\text { (A) } \quad \alpha \mapsto q_{\alpha} \alpha^{\prime}, \quad \text { and } \quad(\mathrm{B}) \quad\left(\alpha^{\prime}\right)^{\vee} \mapsto q_{\alpha} \alpha^{\vee}
$$

Before proceeding we make a reduction. Suppose that the condition (A) on the left holds, and that the quantity $q_{\alpha}$ depends only on whether $\alpha$ is long or short. Then the condition (B) on the right also holds. Indeed under these assumptions the tensor $\sum_{\alpha} \alpha \otimes \alpha$ pulls back under $\hat{N}$ to $q_{1} \sum_{\alpha^{\prime} \text { short }} \alpha^{\prime} \otimes \alpha^{\prime}+q_{2} \sum_{\alpha^{\prime} \text { long }} \alpha^{\prime} \otimes \alpha^{\prime}$ - these are both Weyl invariant 
and (as as both $\hat{H}$ and $\hat{G}^{\sigma}$ are semisimple) positive definite inner products. Using them to identify weights with coweights, we have $\left(\alpha^{\prime}\right)^{\vee}=2 \alpha^{\prime} /\left\langle\alpha^{\prime}, \alpha^{\prime}\right\rangle \mapsto 2 q_{\alpha} \alpha /\langle\alpha, \alpha\rangle=q_{\alpha} \alpha^{\vee}$, as desired.

Now let us prove that (11.4.3)(A) holds. Let $\mathfrak{t}_{G}=X_{*}\left(\mathbf{T}_{G}\right) \otimes \mathbf{R}$. If we identify $\mathfrak{t}_{G}$ with the the Lie algebra of a maximal torus inside the real points of the split form of $\mathbf{G}$, the Killing form endows it with an inner product, and $\sigma$ acts on $\mathfrak{t}_{G}$ and preserves this inner product.

Make the following identifications:

(a) $X^{*}\left(\mathbf{T}_{G}\right) \otimes \mathbf{R}=\mathfrak{t}_{G}^{*} \simeq \mathfrak{t}_{G}$ via Killing form; for each root $\alpha$ let $H_{\alpha} \in \mathfrak{t}_{G}$ be the corresponding element representing the root.

(b) $X_{*}\left(\hat{T}_{G}\right) \otimes \mathbf{R}=X^{*}\left(\mathbf{T}_{G}\right) \otimes \mathbf{R} \simeq \mathfrak{t}_{G}$ via (a).

(c) $X_{*}\left(\hat{T}_{G}^{\sigma}\right) \otimes \mathbf{R} \simeq \mathfrak{t}_{G}^{\sigma} \operatorname{via}(\mathrm{b})$.

(d) $X^{*}\left(\hat{T}_{G}^{\sigma}\right) \otimes \mathbf{R} \simeq\left(X_{*}\left(\hat{T}_{G}^{\sigma}\right) \otimes \mathbf{R}\right)^{*} \simeq \mathfrak{t}_{G}^{\sigma}$ via (c) and the Killing form.

(e) $X_{*}\left(\mathbf{T}_{H}\right) \otimes \mathbf{R} \simeq \mathfrak{t}_{G}^{\sigma}$.

(f) $X^{*}\left(\mathbf{T}_{H}\right) \otimes \mathbf{R}=\left(\mathfrak{t}_{G}^{\sigma}\right)^{*} \simeq \mathfrak{t}_{G}^{\sigma}$ via Killing form again.

(g) $X_{*}\left(\hat{T}_{H}\right) \otimes \mathbf{R} \simeq X^{*}\left(\mathbf{T}_{H}\right) \otimes \mathbf{R} \simeq \mathfrak{t}_{G}^{\sigma}$ via (f).

With these identifications the dual norm $\hat{T}_{H} \rightarrow \hat{T}_{G}^{\sigma}$ corresponds to "multiplication by $p$ " on $\mathfrak{t}_{G}^{\sigma}$. The associated root $\alpha_{*}^{\vee}$ for $\hat{G}$ corresponds to $2 H_{\alpha} /\left\langle H_{\alpha}, H_{\alpha}\right\rangle \in \mathfrak{t}$. Then $\frac{2 f_{\alpha}}{p} \frac{\sum H_{\sigma^{i} \alpha}}{\left\langle H_{\alpha}, H_{\alpha}\right\rangle} \in \mathfrak{t}$ represents a root of $(\hat{G})^{\sigma}$. Pulling back under $\hat{T}_{H} \rightarrow\left(\hat{T}_{G}\right)^{\sigma}$, we get the weight of $\hat{T}_{H}$ represented by

$$
2 f_{\alpha} \frac{\sum H_{\sigma^{i} \alpha}}{\left\langle H_{\alpha}, H_{\alpha}\right\rangle}
$$

On the other hand, the root $\alpha$ gives rise to a root of $\mathbf{H}$, namely $e_{\alpha} \bar{\alpha}$, which is represented by $\frac{e_{\alpha}}{p} \sum H_{\sigma^{i} \alpha} \in \mathfrak{t}_{G}^{\sigma}$. The associated coroot for $\mathbf{H}$ is given by

$$
\frac{2}{p e_{\alpha}\left(\cos ^{2} \theta_{\alpha}\right)} \sum \frac{H_{\sigma^{i} \alpha}}{\left\langle H_{\alpha}, H_{\alpha}\right\rangle}
$$

By our assumption (11.4.1), the weight (11.4.4) is a $p$-power multiple of (11.4.5).

In fact, examining (11.4.2), the multiple is given by $1 / 2$ in the type $A_{2}$ case, and $o_{\alpha}^{-1}$ otherwise. From this we see that the multiple depends only on whether the induced root of $\hat{G}^{\sigma}$ is long or short; in particular, it is constant on Weyl orbits.

11.5. Proposition. Assume that $\mathbf{H}$ is inner and that $\sigma$ is conjugate over $\bar{F}$ to a pinned automorphism of $\mathbf{G}$. Then there is a $\sigma$-dual homomorphism. These conditions hold for each of the following pairs $(\mathbf{G}, \mathbf{H}):(\mathrm{SL}(2 n+1), \mathrm{SO}(2 n+1))$ for $n \geq 1,(\mathrm{SL}(2 n), \mathrm{Sp}(2 n))$ for $n \geq 2$, $(\operatorname{Spin}(2 n+2), \operatorname{Spin}(2 n+1))$ for $n \geq 1,\left(\operatorname{Spin}(8), \mathrm{G}_{2}\right)$ and $\left(\mathrm{E}_{6}, \mathrm{~F}_{4}\right)$.

Proof. In each case $\mathbf{H}$ is inner and (by $\S 11.1$ ) the image of $\Gamma$ in $\operatorname{Out}(\mathbf{G})$ is contained in the subgroup generated by $\sigma$. In this setting the Borel class $\left(\mathbf{B}_{H}, \mathbf{B}_{G}\right)$ is $\Gamma$-admissible.

The dual norm extends by Lemma 11.4 to $\psi_{0}: \hat{H} \rightarrow \hat{G}^{\sigma}$ and we take

$$
{ }^{L} \hat{\psi}: \hat{h} \rtimes \gamma \in{ }^{L} \hat{H} \longrightarrow \psi_{0}(\hat{h}) \rtimes \gamma \in{ }^{L} \hat{G} .
$$

This satisfies the conditions of $\S 8.15$. In fact, in all cases ${ }^{L} \hat{\psi}\left(\rho_{H}^{-}(\gamma)\right)=\rho_{G}^{-}(\gamma)$. That is clear in the cases where $p=2$. For $\left(\operatorname{Spin}_{8}, \mathrm{G}_{2}\right)$ with $p=3$ : The cyclotomic character is valued in $\mathbf{F}_{3}^{*}$, and we must verify that (see (7.5.1)) $\hat{N} \circ\left(\Sigma_{H}^{*} / 2\right)$ and $\Sigma_{G}^{*} / 2$ are equal when evaluated on $\mathbf{F}_{3}^{*}$; it is enough to check that the difference $\Sigma_{G}^{*}-\hat{N} \Sigma_{H}^{*}$ is divisible by 4 , i.e. that $\Sigma_{G}$ and $\Sigma_{H} \circ N$ differ by a multiple of 4 , which can be checked by hand. 


\section{Construction of $\sigma$-DUAL homomorphisms 4: COMPUTATIONS IN REMAINING CASES}

In discussing the remaining examples we observe the following: Suppose that $\psi_{1}$ : $\hat{H} \rightarrow \hat{G}$ has trivial centralizer in $\hat{G}(k)$. Then it extends in at most one way to a morphism ${ }^{L} \hat{H} \rightarrow{ }^{L} \hat{G}$ over $\Gamma_{F}$. This applies in all cases of the following:

12.1. Theorem. A $\sigma$-dual homomorphism exists when $(\mathbf{G}, \mathbf{H})$ or $(\mathfrak{g}, \mathfrak{h})$ is a form of any of the following: (when we specify the Lie algebra, we suppose that $\mathbf{G}$ is simply connected).

(i) $\left(\mathfrak{s o}_{8}, \mathfrak{s l}_{3}\right)$, where $\psi_{1}: \mathrm{SL}_{3} \rightarrow \mathrm{PSO}_{8}$ which is the composition of the Frobenius and the adjoint representation.

(ii) $\left(\mathrm{SL}_{2 n}, \mathrm{SO}_{2 n}\right)$ or $(\mathrm{SO}(2 n), \mathrm{SO}(2 a) \times \mathrm{SO}(2 b))$ with $n=a+b$; where $\psi_{1}$ is the composition of the Frobenius and the standard inclusion.

(iii) $(\mathrm{SO}(2 n+2), \mathrm{SO}(2 a+1) \times \mathrm{SO}(2 b+1))$, where $\psi_{1}: \mathrm{Sp}_{2 a} \times \mathrm{Sp}_{2 b} \hookrightarrow \mathrm{Sp}_{2 a+2 b} \stackrel{\iota}{\hookrightarrow}$ $\mathrm{SO}_{2 n+2}$, and $\iota$ is described below (12.2.1).

(iv) When $(\mathfrak{g}, \mathfrak{h})$ is a form of $\left(\mathfrak{e}_{8}, \mathfrak{s l}_{5}^{2}\right)$. Here ${ }^{L} \hat{\psi}$ is described in detail below.

The proof of all cases is similar, but lengthy. We give only details of the final case $\left(\mathfrak{e}_{8}, \mathfrak{s l}_{5}^{2}\right)$, after describing the morphism $\iota$ from case (iii). Note that in some of the other cases it is necessary to use directly the Theorem of $\S 8.1$, not only its corollary from $\$ 8.15$.

12.2. The map $\mathrm{Sp}(2 n) \rightarrow \mathrm{SO}(2 n+2)^{\text {pinned }}$ in characteristic 2 . We describe the map $\iota$ cited above. Write $Q$ for a quadratic form on the vector space $V=k^{2 n}$, with associated nondegenerate bilinear form $B=\langle-,-\rangle$. Let $q$ be the form on the two dimensional vector space $\left\langle f_{1}, f_{2}\right\rangle$ with $q\left(z_{1} f_{1}+z_{2} f_{2}\right)=z_{1} z_{2}$. We describe an embedding

$$
\mathrm{Sp}(B) \rightarrow \mathrm{SO}(Q \bigoplus q)^{\tau}
$$

where $\tau$ is the identity on $V$ swaps $f_{1}, f_{2}$. For any $A \in \operatorname{Sp}(B)$ there exists a unique linear functional $\ell=\ell_{A} \in V^{*}$ such that $Q(A x)-Q(x)=\ell^{2}$. Write $\ell_{A}(v)=B\left(A v, w_{A}\right)$ for some $w_{A} \in V$. Note that, if we use $B$ to give an isomorphism $V \rightarrow V^{*}$ and thus regard $Q$ as a quadratic form also on $V^{*}$, we have $Q\left(\ell_{A}\right)=Q\left(w_{A}\right)$. After all, $\ell_{A}\left(A^{-1} w_{A}\right)=$ $B\left(w_{A}, w_{A}\right)=0$, so that $Q\left(w_{A}\right)=Q\left(A^{-1} w_{A}\right)=Q\left(\ell_{A}\right)$.

Then there is a unique regular function [17] $D$ on $\operatorname{Sp}(B)$, extending the $\{0,1\}$-valued Dickson invariant on $\mathrm{O}(Q)$, with the property that

$$
D(A)^{2}-D(A)=Q\left(w_{A}\right)^{2}=Q\left(\ell_{A}\right)^{2}
$$

i.e. the Artin-Schreier covering of $\operatorname{Sp}(B)$ defined by this last equation has a canonical splitting. With this in hand, the map (12.2.1) is $A \mapsto \operatorname{Frob}(\tilde{A})$ where $\tilde{A}$ is given by

$$
\tilde{A} v=A v+\ell(v)\left(f_{1}+f_{2}\right), \tilde{A} f_{i}=s f_{i}+(1-s) f_{j}+w,\{i, j\}=\{1,2\} .
$$

and $s=\sqrt{D(A)}, \ell=\ell_{A}, w=w_{A}$ are as above. Note that the map $A \mapsto \tilde{A}$ is not regular, because it involves square roots, but $\operatorname{Frob}(\tilde{A})$ does not.

12.3. Proposition. Suppose that $(\operatorname{Lie}(\mathbf{G}), \operatorname{Lie}(\mathbf{H}))$ is a form of $\left(\mathfrak{e}_{8}, \mathfrak{s l}_{5}^{2}\right)$. Then there is a $\sigma$-dual homomorphism.

Let $\mathbf{G}$ be a form of $\mathrm{E}_{8}$ over $F$, let $\sigma \in \mathbf{G}=\operatorname{Aut}(\mathbf{G})$ be an element of order 5 whose centralizer (denoted $\mathbf{H}$ ) is semisimple. We may find a maximal torus $\mathbf{T} \subset \mathbf{H} \times{ }_{F} \bar{F} \subset$ $\mathbf{G} \times{ }_{F} \bar{F}$ over the algebraic closure of $F$, such that $X^{*}(\mathbf{T})$ is naturally identified with the 
$\mathrm{E}_{8}$ lattice in $\mathbf{R}^{8}$. The simple roots of this may be taken to be the columns of the matrix

$$
\left(\begin{array}{rrrrrrrr}
-1 / 2 & -1 & 1 & 0 & 0 & 0 & 0 & 0 \\
1 / 2 & -1 & -1 & 1 & 0 & 0 & 0 & 0 \\
1 / 2 & 0 & 0 & -1 & 1 & 0 & 0 & 0 \\
1 / 2 & 0 & 0 & 0 & -1 & 1 & 0 & 0 \\
1 / 2 & 0 & 0 & 0 & 0 & -1 & 1 & 0 \\
1 / 2 & 0 & 0 & 0 & 0 & 0 & -1 & 1 \\
1 / 2 & 0 & 0 & 0 & 0 & 0 & 0 & -1 \\
-1 / 2 & 0 & 0 & 0 & 0 & 0 & 0 & 0
\end{array}\right)
$$

Let $\alpha_{i}$ denote the $i$ th column of this matrix - this numbering agrees with the Bourbaki numbering. Let $\alpha_{0}$ denote the highest root:

$$
\alpha_{0}=2 \alpha_{1}+3 \alpha_{2}+4 \alpha_{3}+6 \alpha_{4}+5 \alpha_{5}+4 \alpha_{6}+3 \alpha_{7}+2 \alpha_{8}
$$

Then $\Delta_{H}:=\left\{-\alpha_{0}, \alpha_{8}, \alpha_{7}, \alpha_{6}, \alpha_{1}, \alpha_{3}, \alpha_{4}, \alpha_{2}\right\}$ may be taken to be simple roots of $\mathbf{T}$ on $\mathbf{H} \times_{F} \bar{F}$. A visual aid:

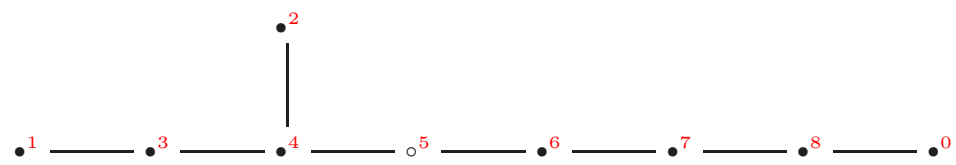

The red numbers denote the indices of the $\alpha \mathrm{s}$, but note the node labeled 0 corresponds to minus $\alpha_{0}$. Let $\mathbf{B}_{H} \subset \mathbf{H} \times_{F} \bar{F}$ denote the Borel subgroup containing $\mathbf{T}$ whose simple roots are the black nodes of the diagram above.

Let us denote by $U$ the following matrix:

$$
U=\left(\begin{array}{rrrrrrrr}
0 & 0 & 0 & 0 & 1 / 2 & -1 / 2 & -1 / 2 & -1 / 2 \\
0 & 0 & 0 & 0 & -1 / 2 & 1 / 2 & -1 / 2 & -1 / 2 \\
0 & 0 & 0 & 0 & -1 / 2 & -1 / 2 & 1 / 2 & -1 / 2 \\
-1 / 2 & -1 / 2 & -1 / 2 & 1 / 2 & 0 & 0 & 0 & 0 \\
1 / 2 & 1 / 2 & -1 / 2 & 1 / 2 & 0 & 0 & 0 & 0 \\
1 / 2 & -1 / 2 & 1 / 2 & 1 / 2 & 0 & 0 & 0 & 0 \\
-1 / 2 & 1 / 2 & 1 / 2 & 1 / 2 & 0 & 0 & 0 & 0 \\
0 & 0 & 0 & 0 & 1 / 2 & 1 / 2 & 1 / 2 & -1 / 2
\end{array}\right)
$$

One checks that $U$ belongs to the Weyl group of $\mathrm{E}_{8}$ - that is, it is orthogonal and preserves the $\mathrm{E}_{8}$-lattice. It moreover has order 4 and preserves $\Delta_{H}$, in particular we have $\left\{1, U, U^{2}, U^{3}\right\} \subset \operatorname{Out}(\mathbf{H})$. Finally, $\left\{1, U, U^{2}, U^{3}\right\}$ is precisely the normalizer of $\mathbf{H}$ in the Weyl group.

Proposition. The map $\Gamma_{F} \rightarrow \operatorname{Out}(\mathbf{H})$ induced by the F-rational structure on $\mathbf{H}$ factors through $\left\{1, U, U^{2}, U^{3}\right\}$. In fact $\gamma$ induces $U^{i}$ exactly when $q_{\gamma}=2^{i}$, where $q_{\gamma} \in \mathbf{F}_{5}^{*}$ is the cyclotomic character.

Proof. The first assertion - that the map factors through $\langle U\rangle-$ follows as before, using (10.2.2). Next, proceed as in Step 1 of Proposition 10.6; with notation as in there, evaluation at $\sigma \in \operatorname{Hom}\left(X^{*}(\mathbf{S}), \mu_{F}\right)$.

Suppose, for example, that $\gamma$ induces $U$. Now $\alpha_{5}$ defines an element of $X^{*}(\mathbf{S})=$ $X^{*}\left(\mathbf{B}_{H}\right)$. The image of $\sigma$ under $\alpha_{5}$ is a primitive 5 th root of unity $\zeta_{5}$. Then $\left(\gamma \alpha_{5}\right)(\sigma)=$ $U \alpha(\sigma)=\zeta_{5}^{2}$ because the coefficient of $\alpha_{5}$ in $U \alpha_{5}$ is 2 , and and $\alpha_{i}(\sigma)=1$ for $i \neq 5$. Similarly $q_{\gamma}=2^{j}$ when $\gamma$ induces $U^{j}$.

To each of element $\gamma \in\left\{1, U, U^{2}, U^{3}\right\}$, we consider a Levi subgroup $\mathbf{L}_{G}(\gamma) \subset \mathbf{G} \times{ }_{F}$ $\bar{F}$ — the centralizer of the identity component ${ }^{6}$ of $\mathbf{T}^{\gamma}$. In fact $\mathbf{L}_{G}(U)=\mathbf{L}_{G}\left(U^{3}\right) \supset$

$6_{\text {though in this case, }} \mathbf{T}^{\gamma}$ is always connected 
$\mathbf{L}_{G}\left(U^{2}\right) \supset \mathbf{L}_{G}(1)=\mathbf{T}$. Let us indicate what the roots of $\mathbf{T}$ on these Levis are. First, put

$$
\begin{aligned}
& x_{1}=\alpha_{2}+\alpha_{3}+\alpha_{4}+\alpha_{5}+\alpha_{6}+\alpha_{7} \\
& x_{2}=\alpha_{1}+\alpha_{2}+\alpha_{3}+2 \alpha_{4}+\alpha_{5} \\
& x_{3}=\alpha_{1}+\alpha_{2}+2 \alpha_{3}+2 \alpha_{4}+2 \alpha_{5}+2 \alpha_{6}+\alpha_{7}+\alpha_{8} \\
& y_{1}=\alpha_{1}+\alpha_{3}+\alpha_{4}+\alpha_{5}+\alpha_{6}+\alpha_{7} \\
& y_{2}=\alpha_{2}+\alpha_{3}+2 \alpha_{4}+\alpha_{5}+\alpha_{6} \\
& y_{3}=-\alpha_{1}-2 \alpha_{2}-2 \alpha_{3}-4 \alpha_{4}-3 \alpha_{5}-3 \alpha_{6}-2 \alpha_{7}-\alpha_{8}
\end{aligned}
$$

The roots generate a root system of type $A_{3} \times A_{3}$ inside $E_{8}$, indeed they are a system of simple roots for such a root system. Computing dot products between these six vectors, we find that their Dynkin diagram is

$$
x_{1}-x_{2}-x_{3} \quad y_{1}-y_{2}-y_{3}
$$

Proposition. We have

(1) $\mathbf{L}_{G}(U)=\mathbf{L}_{G}\left(U^{3}\right)$ is the Levi obtained by adjoining to $\mathbf{T}$ the roots

$$
\begin{array}{llllll} 
\pm x_{1} & \pm x_{2} & \pm x_{3} & \pm\left(x_{1}+x_{2}\right) & \pm\left(x_{2}+x_{3}\right) & \pm\left(x_{1}+x_{2}+x_{3}\right) \\
\pm y_{1} & \pm y_{2} & \pm y_{3} & \pm\left(y_{1}+y_{2}\right) & \pm\left(y_{2}+y_{3}\right) & \pm\left(y_{1}+y_{2}+y_{3}\right)
\end{array}
$$

(2) $\mathbf{L}_{G}\left(U^{2}\right)$ is the Levi obtained by adjoining the roots to $\mathbf{T}$ the roots

$$
\begin{array}{llll} 
\pm x_{1} & \pm y_{3} & \pm y_{1} & \pm y_{3}
\end{array}
$$

In particular the derived subgroups $\left[\mathbf{L}_{G}(U), \mathbf{L}_{G}(U)\right]$ and $\left[\mathbf{L}_{G}\left(U^{2}\right), \mathbf{L}_{G}\left(U^{2}\right)\right]$ are isomorphic to $\mathrm{SL}_{4}^{2}$ and $\mathrm{SL}_{2}^{4}$, respectively. We also record:

(3) If $s_{r}$ denotes the reflection across the hyperplane perpendicular to the root $r$, then

$$
U=s_{x_{1}} s_{x_{2}+x_{3}} s_{-x_{1}-x_{2}} s_{y_{1}} s_{y_{2}+y_{3}} s_{-y_{1}-y_{2}}
$$

In particular $U$ is a Coxeter element of the Weyl group of $\mathbf{L}_{G}(U){ }^{7}$

The roots $x_{1}, x_{2}, x_{3}, y_{1}, y_{2}, y_{3}$ may be extended to a basis of simple roots of $\mathrm{E}_{8}$ in 1720 ways. Let us consider one of them, the following:

$$
\begin{aligned}
\alpha_{1}^{\prime} & =x_{1} \\
\alpha_{2}^{\prime} & =-\alpha_{1}-\alpha_{2}-\alpha_{3}-\alpha_{4}-\alpha_{5}-\alpha_{6} \\
\alpha_{3}^{\prime} & =x_{2} \\
\alpha_{4}^{\prime} & =x_{3} \\
\alpha_{5}^{\prime} & =-2 \alpha_{1}-2 \alpha_{2}-4 \alpha_{3}-5 \alpha_{4}-4 \alpha_{5}-3 \alpha_{6}-2 \alpha_{7}-\alpha_{8} \\
\alpha_{6}^{\prime} & =y_{1} \\
\alpha_{7}^{\prime} & =y_{2} \\
\alpha_{8}^{\prime} & =y_{3}
\end{aligned}
$$

Let us denote the Borel subgroup of $\mathbf{G} \times_{F} \bar{F}$ whose simple roots are $\alpha_{1}^{\prime}, \ldots, \alpha_{8}^{\prime}$ by $\mathbf{B}_{G}$. In what follows, the identification $X^{*}(\mathbf{T}) \simeq X_{*}(\hat{T})$ is understood with respect to $\mathbf{B}_{G}$.

Proposition. The Borel subgroup $\mathbf{B}_{G}$ is $\Gamma$-admissible with respect to $\mathbf{B}_{H}$.

Proof. The row vector $(1,-3,-7,9,7,3,-1,3)$ considered in $X_{*}(\mathbf{T})$ witnesses the admissibility of $\mathbf{B}_{G}$ with respect to $U$ or $U^{-1}$; the vector $(1,-3,-7,8,6,3,0,2)$ witnesses the admissibility with respect to $U^{2}$. Finally one verifies that $\mathbf{B}_{H} \subset \mathbf{B}_{G}$, so it is also admissible with respect to the trivial element.

In what follows, the identification $X^{*}\left(\mathbf{T}_{G}\right) \simeq X_{*}(\hat{T})$ is understood with respect to this Borel $\mathbf{B}_{G}$, and not with respect to the Borel defined by $\alpha_{1}, \ldots, \alpha_{8}$.

The triple $\mathbf{B}_{G} \supset \mathbf{B}_{H} \supset \mathbf{T}$ induces an identification of $\hat{T}_{H}$ with $\hat{T}_{G}$, which extends (uniquely up to $\hat{T}_{H}$-conjugacy) to an injective homomorphism

$$
\psi_{1}^{\prime}: \hat{H} \hookrightarrow \hat{G}
$$

\footnotetext{
${ }^{7}$ As such, in Carter's classification, its conjugacy class in the Weyl group of $\mathrm{E}_{8}$ is the one labeled $\left(2 \mathrm{~A}_{3}\right)^{\prime \prime}$, see Carter, Springer Lecture Notes in Mathematics 131, Table 7
} 
The image of $\psi_{1}^{\prime}$ contains $\hat{T}_{G}$, denote it by $\hat{H}_{1}$. The center of $\hat{H}_{1}$ is isomorphic to $\mu_{5}$ - in particular as $k$ has characteristic 5 , it has a single $k$-point. By Proposition 10.3, it follows that $\psi_{1}^{\prime} \circ$ Frob extends to a homomorphism of $L$-groups ${ }^{L} \hat{H} \rightarrow{ }^{L} \hat{G}$. There is some freedom in choosing this extension, one such extension for each lift of $U \in N_{\mathbf{G}}(\mathbf{T}) / \mathbf{T}=$ $N_{\hat{G}}(\hat{T}) / \hat{T}$ to $\varpi \in N_{\hat{G}}(\hat{T})$ that (1) normalizes $\hat{H}_{1}$ and (2) preserves a pinning of $\hat{H}_{1}$ and (3) has order 4 . Given such a $\varpi_{U}$, we define ${ }^{L} \hat{\psi}$ be the following formula:

$$
{ }^{L} \hat{\psi}(h \rtimes \gamma)=\psi_{1}^{\prime}(\operatorname{Frob}(h)) \varpi_{U}^{j} \rtimes \gamma \text { if } q_{\gamma}=2^{j}
$$

Let us define such a $\varpi=\varpi_{U}$. If $\alpha$ is a root of $\mathbf{T}$ on $\mathbf{G}$, and $\alpha_{*}$ is the corresponding coroot of $\hat{G}$, we let $\iota_{\alpha}$ denote the coroot homomorphism $\iota_{\alpha}: \mathrm{SL}_{2}(k) \rightarrow \hat{G}$. Let $J \in \mathrm{SL}_{2}$ be as in (10.6.4). Then

Proposition. Define $\varphi$ via

$$
\varphi:=\iota_{x_{1}}(J) \cdot \iota_{x_{2}+x_{3}}(J) \cdot \iota_{-x_{1}-x_{2}}(J) \cdot \iota_{y_{1}}(J) \cdot \iota_{y_{2}+y_{3}}(J) \cdot \iota_{-y_{1}-y_{2}}(J)
$$

Let $\varphi$ be as in (12.3.6). Then $\varphi$ has order 8 , normalizes $\hat{H}_{1}$, and lifts $U$; regarded as an element of $\mathrm{SL}_{4} \times \mathrm{SL}_{4}$, its characteristic polynomial is $\left(x^{2}-3\right)\left(x^{2}-2\right)$ on each factor.

Let $r_{1}=\alpha_{3}+\alpha_{4}+\alpha_{7}+\alpha_{8}$ and $r_{2}=\alpha_{1}+\alpha_{2}+\alpha_{3}+\alpha_{4}+\alpha_{6}+\alpha_{7}+\alpha_{8}-\alpha_{0}$. Then $r_{1, *}$ and $r_{2, *}$, regarded as homomorphisms $\mathbb{G}_{\mathrm{m}} \rightarrow \hat{T} \subset \hat{G}$, generate the center of $\hat{L}_{U}$. Let us take

$$
t=\left(-3 r_{1}-r_{2}\right)_{*}(\sqrt{2})
$$

and put $\varpi_{U}=\varphi t=t \varphi$. Now $r_{1}+r_{2}$ differs by $x_{1}+x_{3}+y_{1}+y_{3}$ by an element of $2 X^{*}(T)$. This means that $r_{1, *}+r_{3, *}$ and $x_{1, *}+x_{3, *}+y_{1, *}+y_{3, *}$ take the same value at -1 ; from this we see that $\varpi_{U}^{4}=1$. To verify that ${ }^{L} \hat{\psi}$ is a $\sigma$-dual homomorphism, it suffices to prove that

$$
\rho_{G}^{-}(\gamma) \text { and } \psi_{1}^{\prime}\left(\operatorname{Frob}\left(\rho_{H}^{-}(\gamma)\right)\right) \varpi_{U}^{\log _{2}\left(q_{\gamma}\right)} \text { project to the same element of } \hat{G} \rtimes \gamma / / \hat{L}_{\gamma}
$$

Because $\mathbf{G}$ is inner, it is equivalent to prove that $\psi_{1} \Sigma_{H}^{*}\left(\operatorname{Frob}\left(\sqrt{q_{\gamma}}\right)\right)^{-1} \varpi_{U}^{\log _{2}\left(q_{\gamma}\right)}$ and $\Sigma_{G}^{*}\left(\sqrt{q_{\gamma}}\right)^{-1}$ are conjugate in $\hat{L}_{\gamma}$. Note that $\psi_{1}^{\prime} \Sigma_{H}^{*}=\psi_{1} \Sigma_{H}^{*}$ and, by computing,

$$
\begin{aligned}
\Sigma_{G} & =3 x_{1}+4 x_{2}+3 x_{3}+3 y_{1}+4 y_{2}+3 y_{3}+13 r_{1}+21 r_{2} \\
\psi_{1} \Sigma_{H} & =2 r_{1}+4 r_{2}
\end{aligned}
$$

Let us treat $q_{\gamma}=2$ first. We have $\operatorname{Frob}(\sqrt{2})=-\sqrt{2}$, so we want to check (where $\sim$ denotes $\hat{L}_{\gamma}$-conjugacy)

$$
\psi_{1}\left(\Sigma_{H}^{*}(-\sqrt{2})\right)^{-1} t \varphi \sim \Sigma_{G}^{*}(\sqrt{2})^{-1}
$$

are conjugate in $\hat{L}_{U}$. In particular $\psi_{1} \Sigma_{H}$ (and also $t$ ) is central in $\hat{L}_{U}$, so to show (12.3.8) it is equivalent to show that $t \psi_{1} \Sigma_{H}^{*}(-\sqrt{2})^{-1} \Sigma_{G}^{*}(\sqrt{2})$ is $\hat{L}_{U}$-conjugate to $\varphi^{-1}$. Since $\psi_{1} \Sigma_{H}$ is even in the root lattice, $\psi_{1} \Sigma_{H}^{*}(-\sqrt{2})=\psi_{1} \Sigma_{H}^{*}(\sqrt{2})$. So we are left with showing that

$$
t \frac{\Sigma_{G}^{*}}{\psi_{1} \Sigma_{H}^{*}}(\sqrt{2}) \sim \varphi^{-1}
$$

Note $\Sigma_{H}-\Sigma_{G}=-3 x_{1}-4 x_{2}-3 x_{3}-3 y_{1}-4 y_{2}-3 y_{3}-11 r_{1}-17 r_{2}$. So by (12.3.7) the left-hand side is $\left(3 x_{1}+4 x_{2}+3 x_{3}+3 y_{1}+4 y_{2}+3 y_{3}\right)_{*}(\sqrt{2})$. By a computation in $\mathrm{SL}_{4} \times \mathrm{SL}_{4}$, this is conjugate both to $\varphi$ and $\varphi^{-1}$.

The case $q_{\gamma}=3$ is similar. We now check $q_{\gamma}= \pm 1$. For $q_{\gamma}=1$ there is nothing to check and for $q_{\gamma}=-1$ we must see that $t^{2} \frac{\Sigma_{G}}{\psi_{1} \Sigma_{H}^{*}}(2) \sim \varphi^{-2}$ i.e. the square of the previous 
conjugacy, but now for the smaller group $\hat{L}_{\gamma}$. The left-hand side equals the diagonal matrix $D=\left(\begin{array}{cc}D & \mathbf{0} \\ \mathbf{0} & D^{-1}\end{array}\right)$ in each $\mathrm{SL}_{4}$ factor where $D=\left(\begin{array}{cc}2 & 0 \\ 0 & 3\end{array}\right)$; on the other hand $\varphi^{2}=$ $\left(\begin{array}{cc}-J & \mathbf{0} \\ \mathbf{0} & -J\end{array}\right) \in \mathrm{SL}_{4}$, and indeed both $D$ and $D^{-1}$ are conjugate to $-J$ inside $\mathrm{SL}_{2}(k)$.

\section{REFERENCES}

[1] Avner Ash, Smith theory and Hecke operators, J. Algebra 259 (2003), no. 1, 43-58. MR 1953707 (2003k:11088)

[2] I. N. Bernstein and A. V. Zelevinsky, Induced representations of reductive p-adic groups. I, Ann. Sci. École Norm. Sup. (4) 10 (1977), no. 4, 441-472. MR 0579172 (58 \#28310)

[3] Joseph Bernstein, Course notes of a course on p-adic groups.

[4] A. Borel, Automorphic L-functions, Automorphic forms, representations and $L$-functions (Proc. Sympos. Pure Math., Oregon State Univ., Corvallis, Ore., 1977), Part 2, Proc. Sympos. Pure Math., XXXIII, Amer. Math. Soc., Providence, R.I., 1979, pp. 27-61. MR 546608 (81m:10056)

[5] A. Borel and J. De Siebenthal, Les sous-groupes fermés de rang maximum des groupes de Lie clos, Comment. Math. Helv. 23 (1949), 200-221. MR 0032659 (11,326d)

[6] Armand Borel and Jacques Tits, Groupes réductifs, Inst. Hautes Études Sci. Publ. Math. (1965), no. 27, 55-150. MR 0207712 (34 \#7527)

[7] Nicolas Bourbaki, Éléments de mathématique, Masson, Paris, 1981, Groupes et algèbres de Lie. Chapitres 4, 5 et 6. [Lie groups and Lie algebras. Chapters 4, 5 and 6]. MR 647314 (83g:17001)

[8] _ Algebra II. Chapters 4-7, Elements of Mathematics (Berlin), Springer-Verlag, Berlin, 2003, Translated from the 1981 French edition by P. M. Cohn and J. Howie, Reprint of the 1990 English edition [Springer, Berlin; MR1080964 (91h:00003)]. MR 1994218

[9] K. Buzzard and Toby Gee, The conjectural connections between automorphic representations and Galois representations.

[10] Frank Calegari and David Geraghty, Modularity lifting beyond the Taylor-Wiles method.

[11] Frank Calegari and Akshay Venkatesh, A torsion Jacquet-Langlands correspondence.

[12] P. Cartier, Representations of p-adic groups: a survey, Automorphic forms, representations and $L$-functions (Proc. Sympos. Pure Math., Oregon State Univ., Corvallis, Ore., 1977), Part 1, Proc. Sympos. Pure Math., XXXIII, Amer. Math. Soc., Providence, R.I., 1979, pp. 111-155. MR 546593 (81e:22029)

[13] W. Casselman, Introduction to the theory of admissible representations.

[14] Laurent Clozel, Formes modulaires sur la $\mathbb{Z}_{p}$-extension cyclotomique de $\mathbb{Q}$, Pacific Journal of Mathematics.

[15] P. Deligne and G. Lusztig, Representations of reductive groups over finite fields, Ann. of Math. (2) 103 (1976), no. 1, 103-161. MR 0393266 (52 \#14076)

[16] Pierre Deligne, Letter to J.-P. Serre dated 1 May 2007.

[17] Jean Dieudonné, Pseudo-discriminant and Dickson invariant, Pacific. J. Math. 5 (1955), 907-910. MR $0085212(19,6 a)$

[18] Matthew Emerton, A local-global compatibility conjecture in the p-adic Langlands programme for $\mathrm{GL}_{2 / \mathbb{Q}}$, Pure Appl. Math. Q. 2 (2006), no. 2, Special Issue: In honor of John H. Coates. Part 2, 279-393. MR 2251474 (2008d:11133)

[19] George Glauberman, Correspondences of characters for relatively prime operator groups., Canad. J. Math. 20 (1968), 1465-1488. MR 0232866 (38 \#1189)

[20] Benedict H. Gross, On the Satake isomorphism, Galois representations in arithmetic algebraic geometry (Durham, 1996), London Math. Soc. Lecture Note Ser., vol. 254, Cambridge Univ. Press, Cambridge, 1998, pp. 223-237. MR 1696481 (2000e:22008)

[21] Thomas J. Haines and Sean Rostami, The Satake isomorphism for special maximal parahoric Hecke algebras, Represent. Theory 14 (2010), 264-284. MR 2602034 (2011g:20077)

[22] David Joyner, On finite dimensional representations of non-connected reductive groups, J. Lie Theory 10 (2000), no. 2, 269-284. MR 1774860 (2001h:20060)

[23] Steffen Kionke, On lower bounds for cohomology growth in p-adic analytic towers, Math. Z., to appear.

[24] Robert E. Kottwitz and Diana Shelstad, Foundations of twisted endoscopy, Astérisque (1999), no. 255, vi+190. MR 1687096 (2000k:22024)

[25] R. P. Langlands, Representations of abelian algebraic groups, Pacific J. Math. (1997), no. Special Issue, 231-250, Olga Taussky-Todd: in memoriam. MR 1610871 (99b:11125) 
[26] Martin W. Liebeck and Gary M. Seitz, The maximal subgroups of positive dimension in exceptional algebraic groups, Mem. Amer. Math. Soc. 169 (2004), no. 802, vi+227. MR 2044850 (2005b:20082)

[27] Mark Reeder, Torsion automorphisms of simple Lie algebras, Enseign. Math. (2) 56 (2010), no. 1-2, 3-47. MR 2674853 (2012b:17040)

[28] Peter Scholze, Torsion in the cohomology of locally symmetric varieties.

[29] Jean-Pierre Serre, Cohomologie galoisienne, fifth ed., Lecture Notes in Mathematics, vol. 5, SpringerVerlag, Berlin, 1994. MR 1324577 (96b:12010)

[30] Ekaterina Sopkina, Classification of all connected subgroup schemes of a reductive group containing a split maximal torus, J. K-Theory 3 (2009), no. 1, 103-122. MR 2476042 (2009m:14068)

[31] Robert Steinberg, Regular elements of semisimple algebraic groups, Inst. Hautes Études Sci. Publ. Math. (1965), no. 25, 49-80. MR 0180554 (31 \#4788)

[32] _ The isomorphism and isogeny theorems for reductive algebraic groups, J. Algebra 216 (1999), no. 1, 366-383. MR 1694546 (2000g:20090)

[33] David Treumann, Smith theory and geometric Hecke algebras.

[34] Nikolai Vavilov and Eugene Plotkin, Chevalley groups over commutative rings. I. Elementary calculations, Acta Appl. Math. 45 (1996), no. 1, 73-113. MR 1409655 (97h:20056) 